Projektbericht

Research Report

\title{
Programmevaluierung Jitu
}

Junge, innovative technologierorientierte Unternehmen und i2 - Business Angels

Helmut Gassler und Richard Sellner

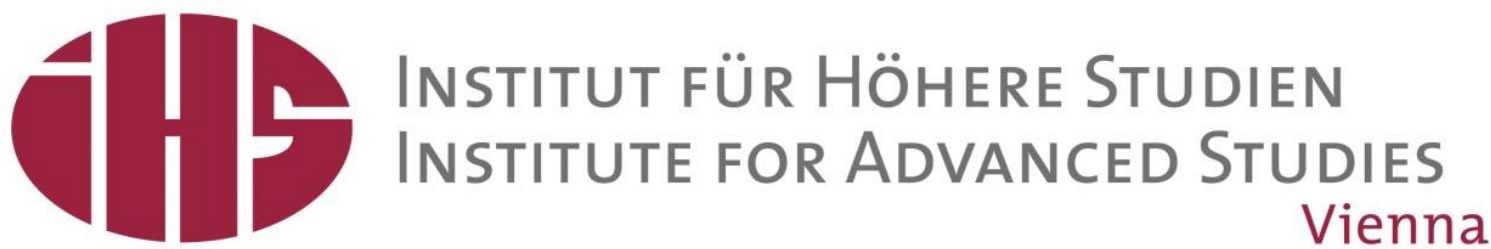



Projektbericht

Research Report

\section{Programmevaluierung Jitu \\ Junge, innovative technologierorientierte Unternehmen und i2 - Business Angels Helmut Gassler und Richard Sellner}

Vorläufiger Endbericht

Studie im Auftrag des austria wirtschaftsservice | erp-fonds

November 2014

Institut für Höhere Studien (IHS), Wien

Institute for Advanced Studies, Vienna 

Kontakt:

Helmut Gassler

용: $+43 / 1 / 599$ 91-249

email: helmut.gassler@ihs.ac.at 



\section{Inhaltsverzeichnis}

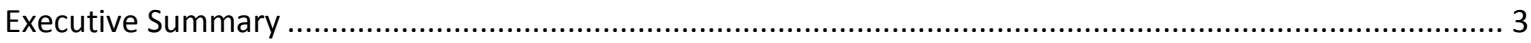

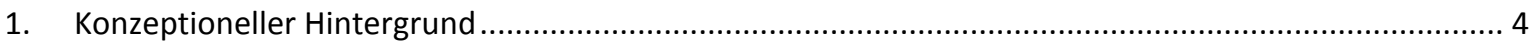

1.1. Zur Bedeutung von technologieorientierten Gründungen ................................................... 4

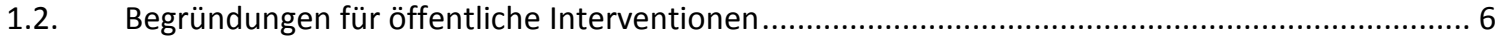

1.3. Gründungsförderung im Kontext einer systemischen Gründungs- und Entrepreneurship-Politik

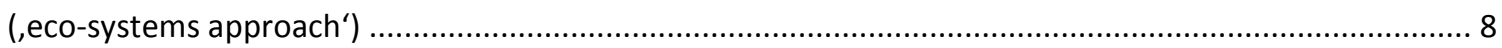

1.4. Exkurs: Finanzierung von High-Tech-Gründungen in Österreich durch Risikokapital.................. 14

1.5. Exkurs: Zum Stellenwert von Business Angels im Gründungsgeschehen Österreichs ................. 17

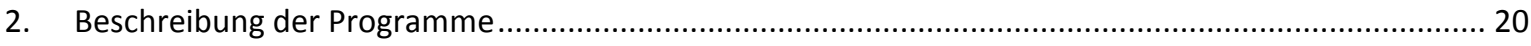

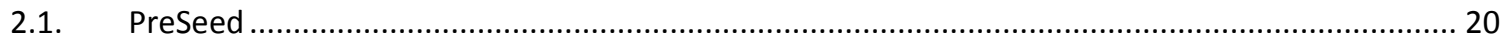

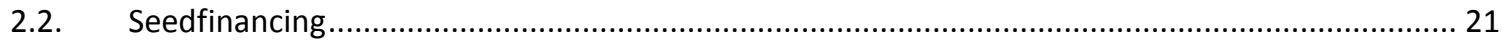

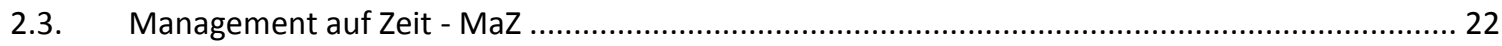

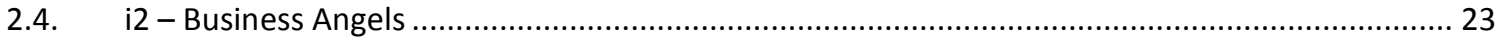

2.5. Spezifika von High-Tech Gründungen und deren Relevanz für die Ausgestaltung des JITUProgramms

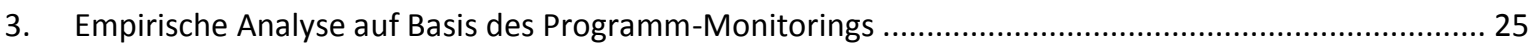

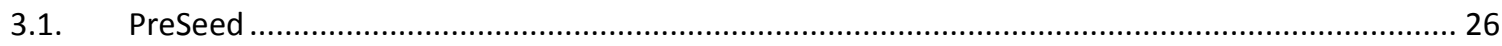

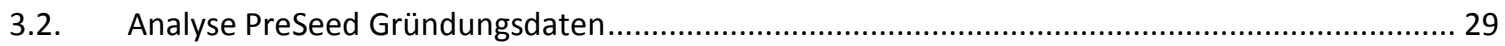

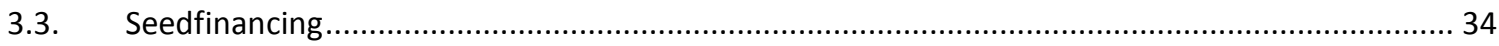

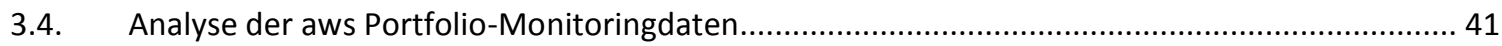

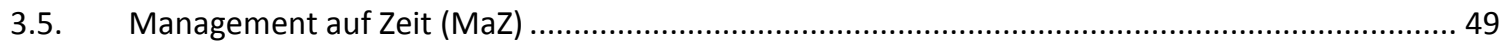

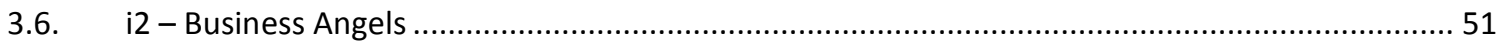

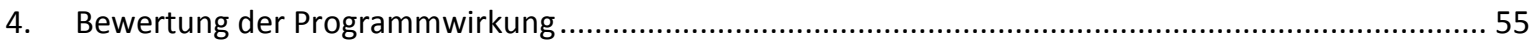

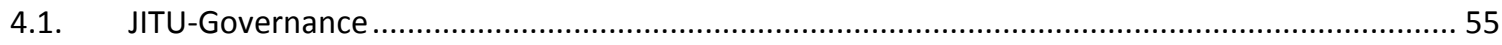

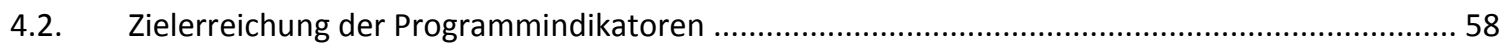

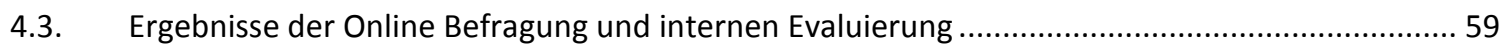

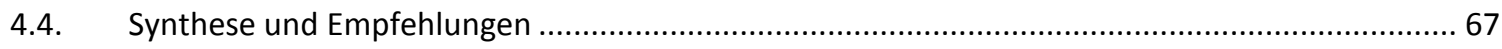

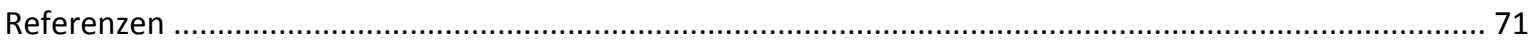

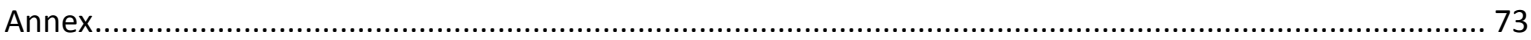





\section{Abbildungsverzeichnis}

Abbildung 1: Wirkungen von High-Tech Gründungen .................................................................. 5

Abbildung 2: Fokussierte Gründungspolitik zur Anhebung der Qualität des Gründungsgeschehens .......... 6

Abbildung 3: Induzierung eines zirkulären, sich selbst verstärkenden Prozesses durch

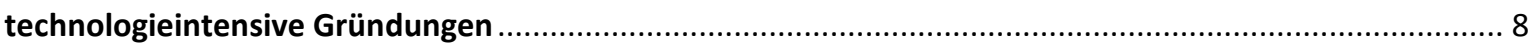

Abbildung 4: Förderinstrumente entlang des Unternehmenszyklus ..................................................... 10

Abbildung 5:Programmkombinationsmöglichkeiten von Förder-, Beratungs- und Awarenessmaßnahmen der aws.

Abbildung 6: Förderkette für innovative Gründungen am Beispiel der Kombination von INiTS-betreuten

Gründungen mit anderen Förderinstrumenten

Abbildung 7: Auszahlungen (Förderungen) an Hightech-Gründungen in Österreich .............................. 13

Abbildung 8: Schematische Darstellung des Start-Up Ökosystems ...................................................... 14

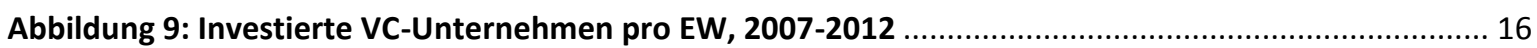

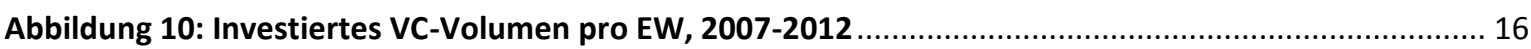

Abbildung 11: Wachstum der Risikokapitalinvestitionen von Business Angels, 2011 auf 2012 ............... 18

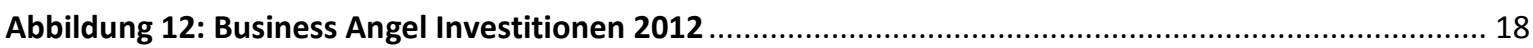

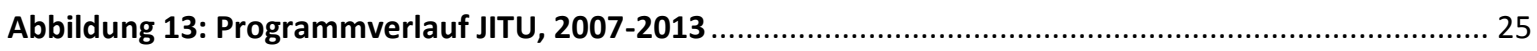

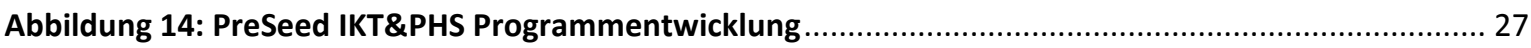

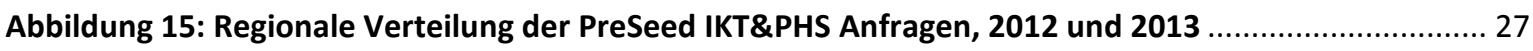

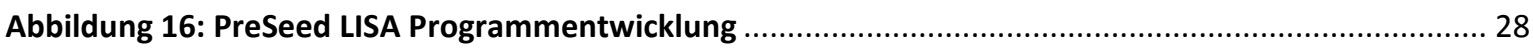

Abbildung 17: PreSeed LISA - durchschnittliche Förderhöhe pro Zusage ........................................... 28

Abbildung 18: Projekte nach Entscheidungsjahr und Programmtyp ..................................................... 29

Abbildung 19: Durchschnittliche Förderhöhe nach Entscheidungsjahr und Programmtyp ....................... 30

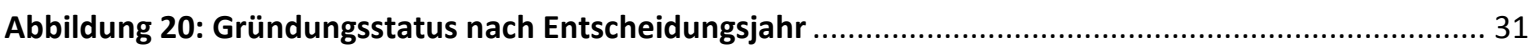

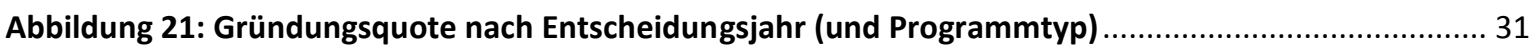

Abbildung 22: Verteilung der Gründungsdauer, insgesamt ........................................................... 32

Abbildung 23: Verteilung der Gründungsdauer nach Programmtyp ...................................................... 32

Abbildung 24: Verteilung der Gründungsdauer nach Entscheidungsjahr ............................................ 33

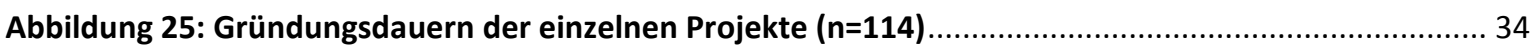

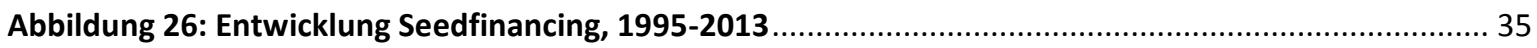

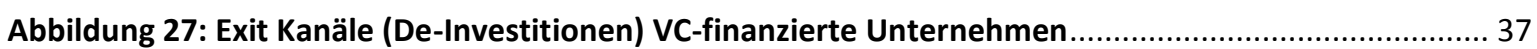

Abbildung 28: Seedfinancing IKT\&PHS Programmentwicklung .............................................................. 39

Abbildung 29: Regionale Verteilung der beschlossenen Seedfinancing Projekte in IKT\&PHS .................. 40

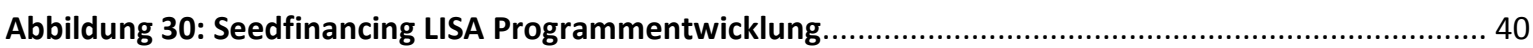

Abbildung 31: Seedfinancing LISA - durchschnittliche Förderhöhe pro Zusage..................................... 41

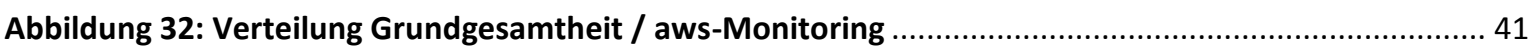

Abbildung 33: Beschäftigungsstand 2011 nach Gründungskohorte ............................................... 42

Abbildung 34: Durchschnittliches Beschäftigungswachstum 2008-2011 nach Gründungsjahr .................. 43

Abbildung 35: Vernetzung der Finanzierung - PreSeed und Seed (LISA und IKT \& Physical Science) ......... 43

Abbildung 36: Vernetzung der Finanzierung - LISA (PreSeed und Seed)............................................. 44

Abbildung 37: Vernetzung der Finanzierung - IKT\&PHS (PreSeed und Seed) .....................................4 44

Abbildung 38: Finanzierungsquellen der geförderten Unternehmen .............................................. 45

Abbildung 39: Förderanteil an der Gesamtfinanzierung und Eigenkapital-Hebel .................................. 46

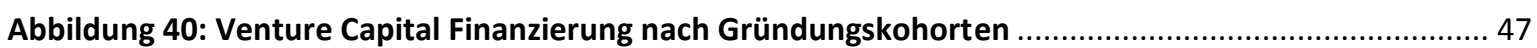

Abbildung 41: Business Angel Finanzierung nach Gründungskohorten.............................................. 48 
Abbildung 42: Patente und F\&E-Ausgaben nach Gründungskohorten

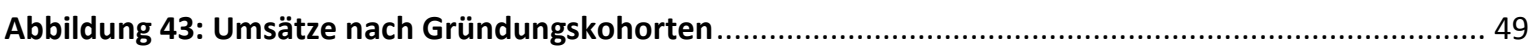

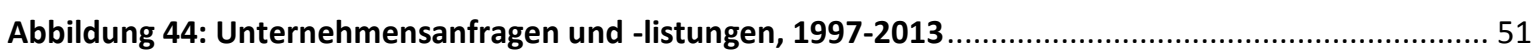

Abbildung 45: Business Angel Listungen und Vermittlungen, 1997-2013 ......................................... 51

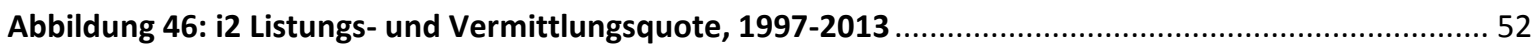

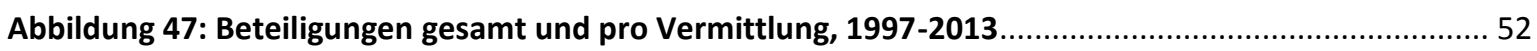

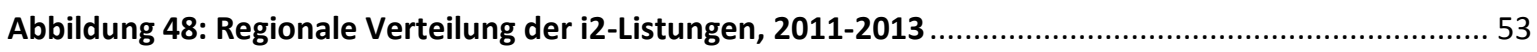

Abbildung 49: Branchenverteilung der i2 Portfoliounternehmen, 2012 ................................................. 54

Abbildung 50: Gründungsmotive (0 wenig Relevanz, 100 sehr hohe Relevanz) .................................. 61

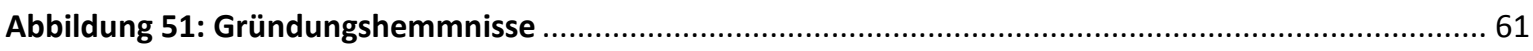

Abbildung 52: Bedeutung PreSeed/Seed für den Gründungsprozess bzw. den Unternehmensaufbau aus

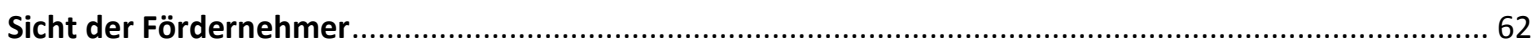

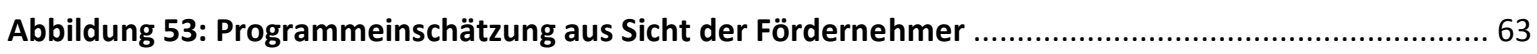

Abbildung 54: Effekt der Förderung auf Finanzierungsbasis im Seedfinancing ..................................... 64

Abbildung 55: Effekt der Förderung auf Projektumsetzung im Seedfinancing ......................................65

Abbildung 56: Projektwirkung im Seedfinancing auf Innovationsaktivitäten und -kompetenzen .............65

Abbildung 57: Teilnahme an aws-Förderung bewirkt ... - eher und sehr vorteilhaft ............................... 66

Abbildung 58: Aus der Projektumsetzung folgt ... - Antwort: Ja ............................................................. 66

Abbildung 59: Das Vorhaben leistet einen Beitrag für ... - Antwort: Großer Beitrag ................................67 67

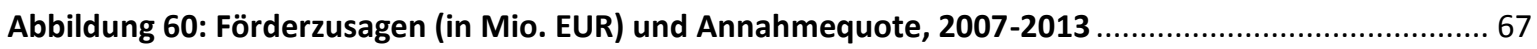




\section{Tabellenverzeichnis}

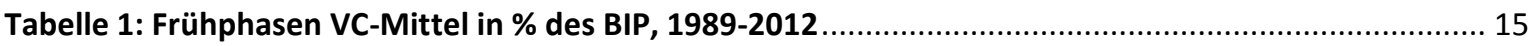

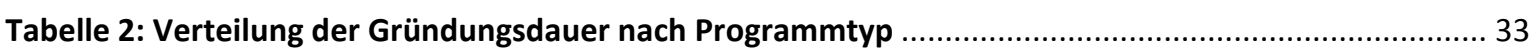

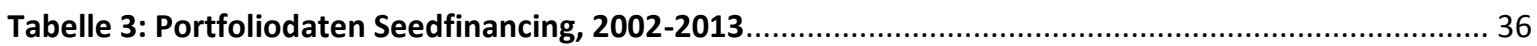

Tabelle 4: Insolvenzraten Unternehmen insgesamt vs. aws geförderte Unternehmen ............................ 36

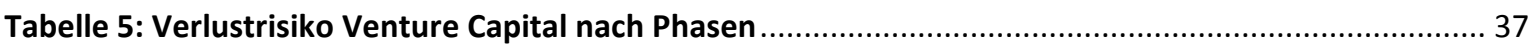

Tabelle 6: Finanzierungsquellen in Prozent und absolut ................................................................... 46

Tabelle 7: Förderanteil an der Gesamtfinanzierung und EK-Hebel, Verteilung ................................... 47

Tabelle 8: Entwicklung Management auf Zeit, 2008-2013 ............................................................ 49

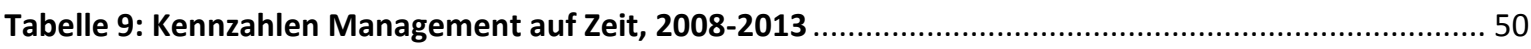

Tabelle 10: i2 Business Angel Investment pro Deal, 1997-2013 ....................................................... 53

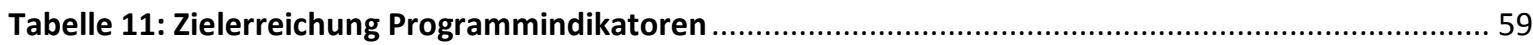

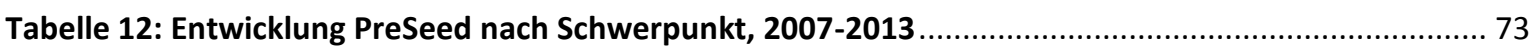

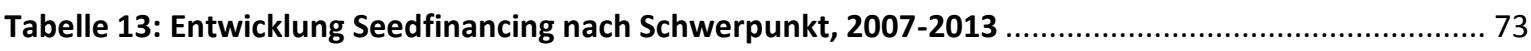

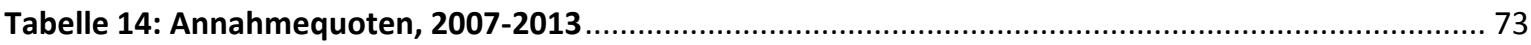

Tabelle 15: PreSeed Gründungen nach Entscheidungsjahr und Programmtyp ...................................... 74

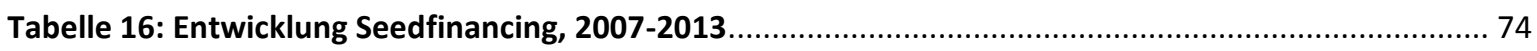

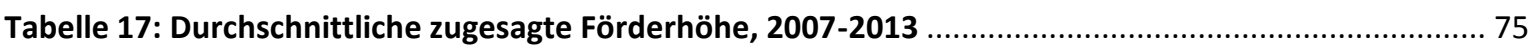

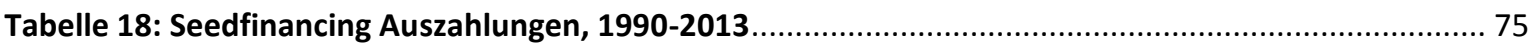

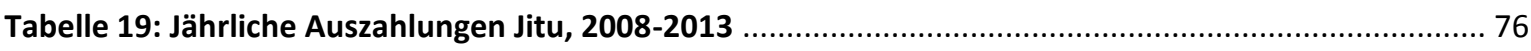

Tabelle 20: Kumulierte Auszahlungen seit Jitu-Programmstart, 2008-2013....................................... 76

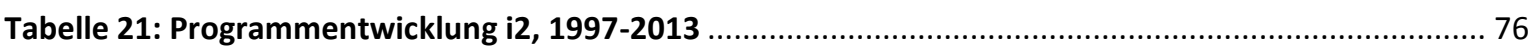

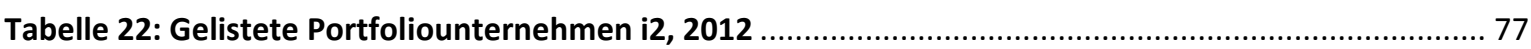

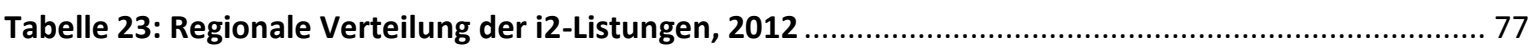





\section{Executive Summary}

JITU ist ein Förderprogramm zur Stimulierung und Finanzierung von jungen technologieorientierten Unternehmen bzw. Gründungen und besteht aus den drei Modulen PreSeed, Seedfinancing und Management auf Zeit. Mit PreSeed werden potenzielle UnternehmensgründerInnen hinsichtlich der Überprüfung der Machbarkeit und Kommerzialisierbarkeit ihrer technologieorientierten Geschäftsideen mit bis zu 200.000 Euro unterstützt. Das Programmmodul Seedfinancing adressiert mit bis zu einer Mio. Euro die Finanzierung des Unternehmensaufbaus bzw. die Umsetzung der technologieorientierten Geschäftsidee in marktfähige Produkte und Leistungen. Unterstützend wird als drittes Modul Management auf Zeit (MaZ) angeboten, mit dem Seedfinancing geförderte Unternehmen auf temporärer Basis externe personelle Expertise einholen können. Gemäß dem Programmdokument zu den JITU-Richtlinien ist am Ende der Programmperiode (31. Dezember 2014) eine abschließende Evaluierung vorzulegen. Darüber hinaus war es auch Aufgabe dieser Studie das aws Programm „,i2 - die Börse für Business Angels“ zu evaluieren. i2 fungiert als Vermittlungsplattform zwischen privaten Investoren (Business Angels) und kapitalsuchenden Unternehmen.

Die vorliegende Evaluierung basiert auf den internen Programmdaten der aws, auf vorhandenen Ergebnissen eines programmbegleitenden Monitorings, aws internen Evaluierungen und Auswertungen, sowie auf umfangreichen qualitativen Analysen (Interviews mit aws Programmverantwortlichen, Stakeholdern und Fördernehmern). Die empirischen Analysen sind dabei in einem konzeptionellen Rahmen eingebettet, der davon ausgeht, dass das JITU-Programm innerhalb in einem Ökosystem bestehend aus unterschiedlichen Akteuren und Institutionen eingebettet ist.

Als Ergebnis der Evaluierung kann festgehalten werden, dass das JITU-Programm von seiner Konzeption und seinem Instrumentarium her in geeigneter Art und Weise die in den Programmrichtlinien festgelegten Ziele (Steigerung von technologieorientierten Gründungen, Steigerung der Überlebensquote, Ermöglichen einer Anschlussfinanzierung von Risikokapital) adressiert. Die quantitativen Zielindikatoren werden in allen drei JITU Modulen de facto durchgängig erreicht bzw. tlw. übertroffen. Das JITU-Programm ist daher ein zentrales Element des österreichischen Start-Up Ökosystems und verfügt über zahlreiche komplementäre Schnittstellen zu anderen Gründungs- und Technologieförderungen. Durch die kontinuierliche Existenz des Namen "Seedfinancing" konnte das Programm in der Gründerszene einen hohen Bekanntheitsgrad erlangen und gilt heute gleichsam als „Markenname“ für eine technologieorientierte Gründungsförderung, die auch international eine hohe Sichtbarkeit und „Good Practice“ Charakter aufweist.

Die Evaluierungsempfehlungen für das JITU-Programm gehen in zwei Richtungen. Quantitativ ist eine monetäre Aufstockung der Fördermittel vor dem Hintergrund des HighTech-Gründungsaufkommens ohne Qualitätsabstriche bei den Förderprojekten möglich. Qualitative Empfehlungen betreffen vor allem Aspekte der Governance des Programms wie zum Beispiel die Sicherstellung, dass Ausführungsverträge beim Übergang zwischen den jeweiligen 3-Jahres-Verträgen rechtzeitig vorliegen, die Ausgestaltung der Entscheidungsgremien oder den Grad der operativen Entscheidungsautonomie der aws.

Die Ergebnisse Evaluierung des Programms i2 zeigen eine durchwegs positive Entwicklung dieses Programms. Insbesondere in den letzten drei Jahren kam es zu einer Übererfüllung der quantitativen Zielindikatoren. Es konnte mittlerweile eine kritische Masse an Business Angels aktiviert werden, was wiederum bereits positive Effekte auf die Zahl der erfolgreichen Vermittlungen nach sich gezogen hat. Ein konkreter Handlungsbedarf in Bezug auf die Ausgestaltung dieses Programms ist nicht gegeben. 


\section{Konzeptioneller Hintergrund}

\subsection{Zur Bedeutung von technologieorientierten Gründungen}

Das Interesse der Wirtschaftswissenschaft wie auch der Wirtschaftspolitik an Unternehmensgründungen und ihrer Rolle für eine dynamische Wirtschaftsentwicklung hat in den letzten Jahren stark zugenommen. Dies stellt insofern einen Paradigmenwandel dar, da die Diskussion lange Zeit vom Konzentrationsprozess wirtschaftlicher Aktivitäten auf Großunternehmen geprägt war. Ab etwa Anfang der 1990er Jahre fand allerdings dann zunehmend nicht mehr das bürokratische Großunternehmen, sondern das flexible, innovative Kleinunternehmen an Beachtung. Heute herrscht weitgehend darüber Einigkeit, dass Unternehmensgründungen insbesondere in Bezug auf folgende Themenkomplexe eine große Bedeutung aufweisen:

\section{Wettbewerbswirkung}

Der Stellenwert, den Neugründungen für das Offenhalten der Märkte und somit für die Aufrechterhaltung eines effizienten Wettbewerbsdrucks einnehmen, ist in der Industrieökonomie prinzipiell unumstritten: „An excess level of profitability induces entry into the industry. And this is why the entry of new firms is interesting and important - because the new firms provide an equilibrating function in the market, in that the levels of price and profit are restored to the competitive levels". (Audretsch 1995, S. 39)

\section{Beschäftigungswirkung}

Die absolute und relative Bedeutung von Neugründungen für das Beschäftigungswachstum ist unbestritten. Empirische Untersuchungen zeigen, dass ein beträchtlicher Teil des Beschäftigungswachstums auf neugegründete bzw. junge Kleinunternehmen zurückzuführen ist. Durch die Arbeiten von Birch (1981) in den 1980er Jahren wurde geradezu ein Paradigmenwechsel ausgelöst, stand doch bis zu diesem Zeitpunkt mehr die Entwicklung (industrieller) Großunternehmen im Vordergrund, denen für nahezu alle Bereiche unternehmerischer Tätigkeiten gravierende Wettbewerbsvorteile gegenüber kleinen (und neugegründeten) Unternehmen eingeräumt wurden. Mittlerweile konnte vielfach gezeigt werden, dass kleine, aber schnell wachsende Unternehmen („Gazellen“) in hohem Ausmaß zum Beschäftigungswachstum beitragen.

\section{Strukturwandel}

Der Drei-Sektoren-Hypothese zur Folge wird mit zunehmender Höherentwicklung einer Volkswirtschaft zunächst eine Verlagerung der wirtschaftlichen Dynamik vom Primären (Land- und Forstwirtschaft, Bergbau) zum Sekundären Sektor („Industrialisierung“) und in weiterer Folge auf den Tertiären Sektor („Tertiärisierung“) unterstellt. In den letzten Jahren wird zusätzlich von einem „quartären Sektor“ gesprochen, der im Wesentlichen komplexe, informationsgenerierende und -verarbeitende Aktivitäten umfasst („wissensintensive Dienstleistungen“). Unternehmensneugründungen tragen zu diesem Prozess des Strukturwandels aktiv bei. Vielfach finden sich Unternehmensgründungen gerade in den dynamischen, wachsenden Sektoren einer Volkswirtschaft. Der Anteil der Neugründungen ist gerade in den dynamischen Bereichen des Dienstleistungssektors wesentlich höher als der entsprechende Anteil Gesamtbestand. Neugründungen nehmen somit eine aktive Rolle im strukturellen Wandel in Richtung Tertiärisierung und zunehmender Wissensorientierung ein.

Innovation und technologischer Wandel

Bereits im Zuge der späten 70er und frühen 80er Jahre gewann die Frage nach der Rolle, die Unternehmensgründungen im Innovationsprozess bzw. im technologischen Wandel spielen, zunehmendes Interesse. Die nicht zu unterschätzende Rolle, die kleine bzw. neugegründete Unternehmen im Innovationsprozess bzw. im technologischen Wandel spielen, wurde in den vergangenen Jahren immer 
wieder bestätigt. Unternehmensgründungen im Hochtechnologiesektor zeitigen dabei sowohl direkte als auch indirekte Wirkungen. Einerseits tragen sie durch ihre innovativen Geschäftsmodelle bzw. durch ihre technologischen Entwicklungen (in Form von neuen Produkten und/oder Prozessen) unmittelbar zum technologischen Wandel bei. Und andererseits zwingen sie durch ihren Markteintritt bestehende Unternehmen ihre Produktpalette kontinuierlich zu überdenken und gegebenenfalls anzupassen. Dadurch tragen sie zur generellen Hebung der Innovationsintensität einer Volkswirtschaft bei.

In Summe die sind unterschiedlichen Effekte von Unternehmensgründungen in Abbildung 1 dargestellt und es zeigt sich, dass Unternehmensgründungen eine wichtige Rolle zur Verhinderung der „Verkrustung“ von Wirtschaftsstrukturen sowie zum Aufgreifen neuer Marktfelder spielen.

\section{Abbildung 1: Wirkungen von High-Tech Gründungen}

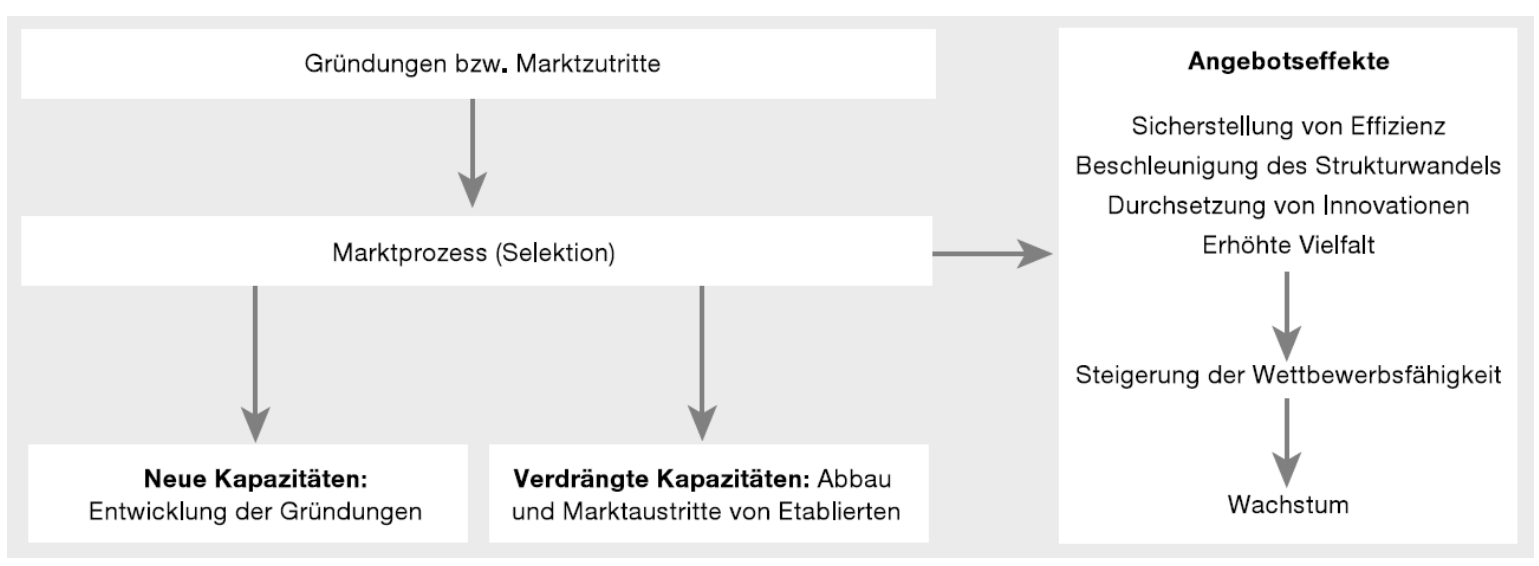

Quelle: Fritsch (2008).

Diese dargestellten positiven Effekte von (technologieorientierten bzw. innovativen) Gründungen und ihre Rolle im Strukturwandel und bei der Beschäftigungsgenerierung macht sie zu einem wichtigen Adressaten der Wirtschaftspolitik. In de facto allen OECD-Staaten ist in den vergangenen Jahren bzw. Jahrzehnten ein vielfältiges Instrumentenportfolio zur Förderung von Unternehmensgründungen entwickelt worden. Eine ausschließliche Orientierung auf eine Erhöhung der Bruttogründungsrate ist daher nicht angebracht, da eine hohe Anzahl von Gründungen und Schließungen sorgt u.U. für ineffiziente Turbulenzen in der Unternehmenspopulation. Die Gründung von Unternehmen schlechter Qualität und in Branchen, die geringe Wachstumschancen bieten führt letztlich vor allem zu hohen Schließungsraten und damit zur Vergeudung von volkswirtschaftlichen Ressourcen. Als Folge dessen betont die neuere Literatur zur Entrepreneurship-Politik die Differenzierung bzw. Selektion der Gründungsprojekte nach der Qualität. Abbildung 2 zeigt eine derartige stilisierte Verteilung. 


\section{Abbildung 2: Fokussierte Gründungspolitik zur Anhebung der Qualität des Gründungsgeschehens}

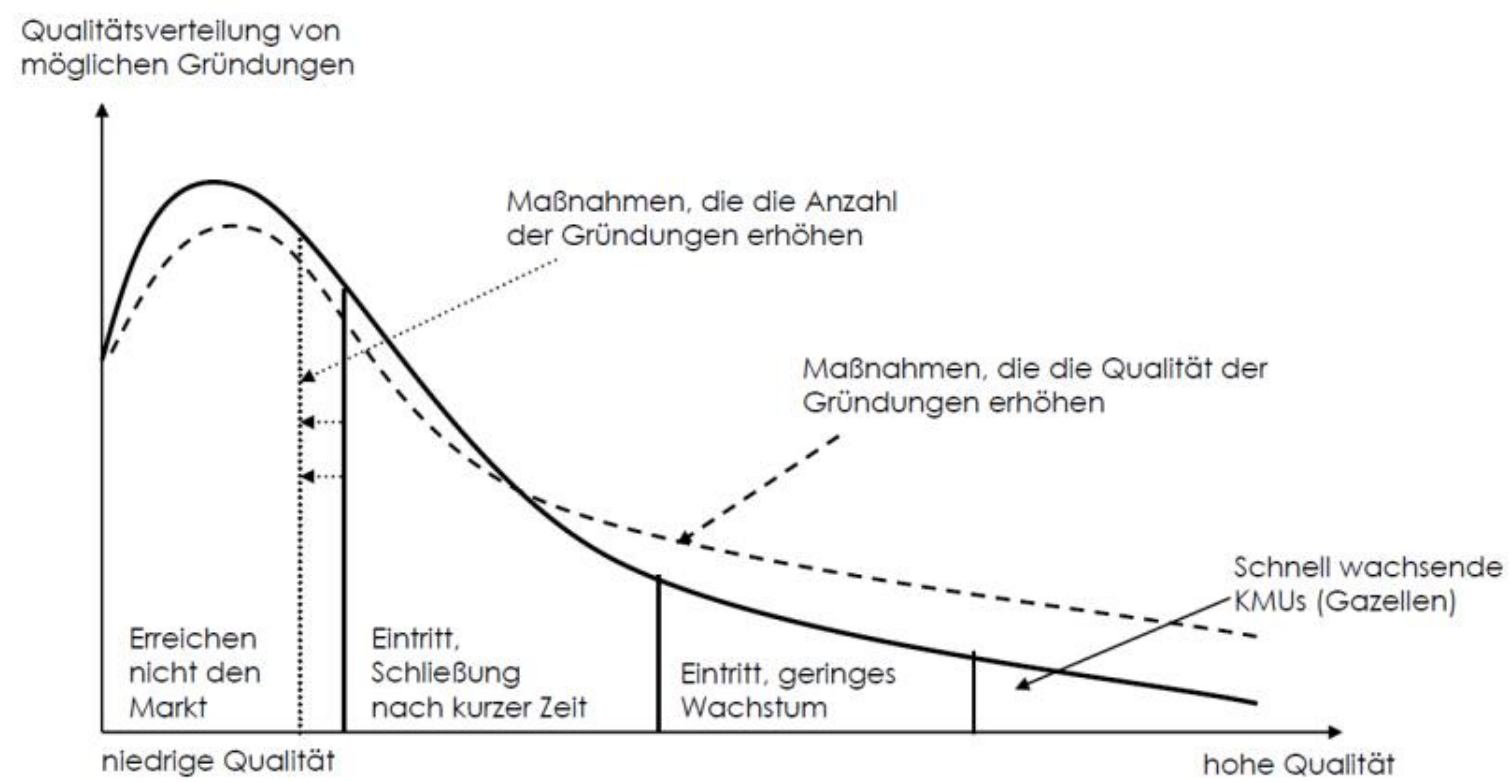

Quelle: Hölzl et al. (2007).

Die durchgezogene Linie zeigt den (angenommenen) Ist-Zustand, der sich vor allem durch eine hohe Konzentration von Gründungen geringer Qualität und einen sehr geringen Anteil von hochwertigen Gründungen und einem hohen Anteil an temporären Gründungen (Turbulenz) auszeichnet. Unter Qualität sind das Potential zu Beschäftigungs- und Produktivitätswachstum („Skalierbarkeit“ der Geschäftsidee), positive Beiträge zum technologischen und strukturellen Wandel und eine höhere Nachhaltigkeit der Gründung (hohe Überlebensrate) zu verstehen. Nachdem vor allem qualitativ hochwertige Gründungsprojekte die oben dargestellten positiven volkswirtschaftlichen Effekte erfüllen, während Gründungen niedriger Qualität nur geringe positive Effekte zeigen, sollte eine moderne Gründerpolitik vor allem auf eine Veränderung der Verteilung der Qualität der Unternehmensgründungen abzielen. Im konkreten Fall bestünde etwa die Aufgabe der aws und des ERP-Fonds in der Steigerung der Häufigkeit von qualitativ hochwertigen Gründungen, was einer Verschiebung der Kurve am rechten Rand der Verteilung nach oben gleichkommt (gestrichelte Linie).

Die Fokussierung der Gründungspolitik auf Qualität betont insbesondere Shane (2009): “Getting economic growth and jobs creation from entrepreneurs is not a numbers game. It is about encouraging the formation of high quality, high growth companies."

Gemäß den Zielen der JITU-Gründungsförderung (Forcierung technologisch anspruchsvoller und innovativer Gründungen mit Wachstumspotential) erfüllt das Programm diesen Anspruch einer Betonung von qualitativ überdurchschnittlichen Gründungen.

\subsection{Begründungen für öffentliche Interventionen}

Als theoretische Begründung für die Förderung von (technologieintensiven) Unternehmensgründungen mit öffentlichen Mitteln wird zum einen die Existenz von Marktversagen bei der externen Finanzierung von jungen Unternehmen herangezogen und zum anderen, die Argumentation, dass insbesondere technologieintensive und innovative Gründungen externe Effekte (spillovers) auslösen.

\section{Zum Marktversagen am Kapitalmarkt}

Hinsichtlich der externen Finanzierung auf Kapital- und Kreditmarkt für junge, innovative Unternehmen wird von einem Marktversagen ausgegangen. Zwischen Kapitalgebern und Kapitalnehmern bestehen 
Informationsasymmetrien, welche zu einer adversen Selektion führen und in ein Moral-Hazard-Problem zur resultieren. Letzteres bezieht sich auf die Tatsache, dass der Kapitalgeber nach Vergabe des Kapitals an den Kapitalnehmer kein perfektes Monitoring über dessen Sorgfalt und Einsatzbereitschaft vornehmen kann:

- $\quad$ Bei jungen bzw. kleinen Unternehmen ist der Kapitalbedarf üblicherweise nicht sehr hoch. Das führt allerdings zu hohen Fixkosten der Risikobewertung sowie anderer Transaktionskosten relativ zum benötigten Kreditvolumen. Für den Kapitalgeber ist daher die Vergabe eines Kredits an derartigen Unternehmen vergleichsweise unattraktiv.

- Neugegründete bzw. junge Unternehmen können nur geringe Sicherheiten bieten und verfügen auch noch über keinen track record bezüglich ihrer „Kredithistorie“ auf, d.h. sie können nicht nachweisen, dass sie auch bereits in der Vergangenheit externe Kapitalmittel pünktlich bedienen konnten.

- Gerade in den ersten Jahren nach erfolgter Gründung ist ein hohes Risiko eines Marktaustritts gegeben. Diese hohen Sterbequoten in den frühen Jahren der Unternehmensgeschichte resultieren ein entsprechendes Risiko für den Kreditgeber, was zu einer Kreditrationierung durch die Bank führt.

Diese allgemeinen Probleme verschärfen sich bei Gründungen mit hoher Technologie- und Innovationsintensität: Die technologische Komplexität der Geschäftsidee erhöht generell die Informationsasymmetrie und erschwert dadurch Risikoeinschätzung. Letztlich verfügen die Banken nur in unzureichendem Ausmaß über das Know-how die technologischen Risiken von innovativen Projekten einzuschätzen. Innovative Unternehmen verfügen außerdem üblicherweise (vor allem in den Frühphasen der Unternehmensentwicklung) über wenig tangible Vermögensgüter auf, die als Sicherheiten herangezogen werden könnten. Darüber hinaus besteht ein Großteil der für den Unternehmensaufbau notwendigen F\&E-Aufwendungen bzw. Innovationsanstrengungen aus Personalkosten. Diese können im Gegensatz zum herkömmlichen Kapitalstock in Form von Anlagen und Maschinen nicht liquidiert werden.

Ohne wirtschaftspolitische Eingriffe von Seiten der öffentlichen Hand kommt es somit zu einer gesamtwirtschaftlich suboptimalen Allokation von Kapital für technologieintensive und innovative Gründungen was letztlich zur Folge hat, dass sich aufgrund einer zu geringen Gründungstätigkeit in diesem Bereich die in Kapitel 1.1 diskutierten positiven Effekte des technologieorientieren Gründungsgeschehens nicht entfalten können.

\section{Zur Rolle von externen Effekten}

Durch die positiven externen Effekte (Spillovers) kommt es zu einem steigenden Informations- und Wissenspool, Agglomerationseffekte entstehen und die Standortattraktivität erhöht sich insgesamt. Diese verbesserte Standortattraktivität induziert dann wiederum neue Gründungsaktivitäten und ein zirkulärer, sich selbst verstärkender Prozess entfaltet sich (vgl. Abbildung 3). 
Abbildung 3: Induzierung eines zirkulären, sich selbst verstärkenden Prozesses durch technologieintensive Gründungen

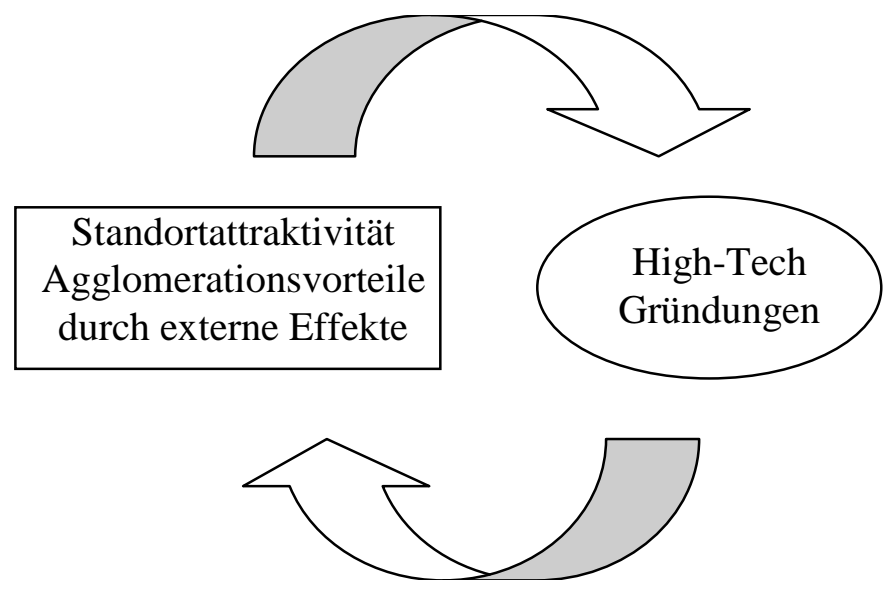

Quelle: eigene Darstellung.

Diese positiven externen Effekte betont unter vielen anderen z.B. Lerner (2009): „In the activities associated with entrepreneurship and venture capital, the actions of any one group are likely to have positive spillovers (...) for their peers." Durch die hohe Gründungstätigkeit entsteht auch ein Markt für spezialisierte Dienstleistungen (z.B. auf Gründungen bzw. junge Unternehmen spezialisierte Steuerberater etc.) und private VC-Fonds werden attrahiert. Im Idealfall entwickelt sich eine lebendige Startup-Szene, die erfolgreichen Gründer geben ihr Knowhow weiter, werden als „serial entrepreneurs“ aktiv oder finanzieren dann die nächste Welle an Gründungen. Archetypisch für diesen zirkulären Prozess ist die Entwicklung des Silicon Valley, das sich innerhalb nur einiger Jahrzehnte von einer landwirtschaftlich strukturierten Region zum weltweiten Zentrum der IKT-Industrie wandelte.

\subsection{Gründungsförderung im Kontext einer systemischen Gründungs- und Entrepreneurship-Politik (,eco-systems approach') $)^{1}$}

In der Gründungsförderung wird mittlerweile eine Vielzahl ganz unterschiedlicher Typen von Programmen zur Stimulierung des Gründungsgeschehens angewandt. Diese einzelnen Typen lassen sich wie folgt charakterisieren:

- Programme zur Unterstützung der Gründungsfinanzierung: Zum Einsatz kommen Instrumente wie direkte (verlorene) Zuschüsse, zinsgestützte Darlehen, Garantien (Haftungsübernahme von privaten Bankkrediten des Gründers) oder die Bereitstellung von (öffentlichen) Risikokapital bzw. die Vergabe von eigenkapitalähnlichen Darlehen (Mezzanin). Die Bereitstellung von Risikokapital durch die öffentliche Hand hat in den letzten Jahren an Bedeutung gewonnen und auch in HORIZON 2020 wird dieses Instrument eine wichtige Rolle spielen. Neben diesen direkten Instrumenten finden sich auch spezifische steuerliche Begünstigungen für junge Unternehmen (z.B. bei der Körperschaftssteuer) oder Begünstigungen hinsichtlich der Sozialversicherung, die letztlich die Finanzierung eines jungen Unternehmens erleichtern.

- Projektbezogene Förderung für F\&E-Projekte: Gerade technologieorientierte Gründungen benötigen für die Umsetzung ihrer Geschäftsidee oft umfangreiche F\&E-Anstrengungen. Somit hat für diese Gründungen, die projektbezogene Förderung der Angewandten Forschung eine hohe Relevanz und erleichtert damit die Finanzierung des Unternehmensaufbaus.

\footnotetext{
${ }^{1}$ Die Argumentation dieses Kapitels folgt im Wesentlichen Schibany et al. (2013).
} 
- Bereitstellung von Beratungsleistungen: Derartige Maßnahmen (z.B. rechtliche Beratung, Beratung bezüglich des Förderangebots, Beratung bezüglich Erstellung des Business Plans, betriebswirtschaftliche Beratung) zielen darauf ab das Informationsdefizit der Gründer zu verringern.

- Bereitstellung technischer Infrastruktur: In Gründerzentren (bzw. neuerdings in sogenannten CoWorking-Spaces) werden Büroräume zu günstigen Konditionen zur Verfügung gestellt und oft auch zusätzlich Gemeinschaftseinrichtungen wie Seminar- und Meetingräume, IKT-Infrastruktur etc.

- Maßnahmen zur Erhöhung der Awareness und Darstellung von „Role Models“: Zur Attraktivierung von „entrepreneurship“ bzw. von Unternehmensgründung als eine berufliche Option finden sich vielfältige Instrumente wie z.B. Verleihung von Awards bzw. Preisen („Gründer des Jahres“ etc.), Medienkampagnen, die Durchführung von Business Plan Wettbewerben oder die Veranstaltung von Netzwerktreffen (Startup Conventions).

- Ausbildungs- und Trainingsmaßnahmen: Darunter fallen zum einen einschlägige Schulungen in speziellen Bereichen (z.B. Buchhaltung und Controlling, Marketing, Handelsrecht etc.), etwa in Form von spezifisch zugeschnittener Kurse (unterschiedlicher Dauer) und zum anderen die Anhebung des Ausbildungsniveaus bezüglich entrepreneurship auf unterschiedlichen Bildungsebenen (z.B. durch, virtuelle Unternehmen' in Schulen der Sekundarstufe oder durch spezielle Curricula im Bereich entrepreneurship in der tertiären Bildungsebene).

- Netzwerk- bzw. Clusterbildung: Synergieeffekten können etwa durch die Kombination unterschiedlicher Maßnahmen unter einem Programmschirm erzielt werden (z.B. Begleitung von monetären Fördermaßnahmen durch sogenannte, wirkungserhöhende' Maßnahmen im Bereich von Awareness, Ausbildung, Training etc.). Daneben können durch thematische Clusterbildung (z.B. Life Sciences) spillover-Effekte zwischen den Gründungen (aber auch zwischen Gründungen und bestehenden Unternehmen) erzielt werden (z.B. durch „Gründertreffen“ etc.). Zusätzlich wird durch eine derartige thematische Clusterbildung auch die ,Sichtbarkeit' und Attraktivität eines Standorts für ein bestimmtes Technologiefeld verbessert.

Die konkrete Ausgestaltung des Systems der (technologie- und innovationsorientierten) Gründerförderung in Österreich auf Bundesebene wird in Abbildung 4 dargestellt. Dabei werden die unterschiedlichen Förderprogramme der entsprechenden Phase im Gründungsprozess zugeordnet.

In der Frühphase der Unternehmensentwicklung treten bei technologieorientierten Gründungen aufgrund der Notwendigkeit von F\&E-Ausgaben (Proof of Concept, Labortests, technologische Umsetzung der Geschäftsidee in Form eines Prototypen etc.) hohe Kosten bei gleichzeitig noch geringen (bzw. oft auch noch fehlenden) Umsätzen auf. Gleichzeitig besteht aufgrund Unsicherheiten über Technologie und eine Akzeptanz auf dem potentiellen Markt ein hohes Risiko des Scheiterns, was dieser Phase auch die Bezeichnung „Valley of Death" beigebracht hat. Aus diesen Gründen kommen hier für die Unternehmensfinanzierung neben Eigenmittel (oft in Verbindung mit den so genannten „3F“ (Family, Friends and Fools) nur Risikokapitalgeber (in Form von Business Angels und/oder auf Frühphase spezialisierte VC-Fonds, wobei anzumerken ist, dass VC-Fonds vielfach erst später einsteigen, z.B., wenn die Skalierbarkeit der Geschäftsidee tatsächlich bereits absehbar ist) sowie die öffentliche Hand in Betracht. Externe Finanzierungen durch Bankkredite sind in dieser Phase fast ausgeschlossen. Das JITU-Programm füllt somit genau diese Finanzierungslücke in der Frühphase, indem es mit der Schiene PreSeed die formale Unternehmensgründung erleichtert und dann mit der Schiene Seedfinancing dazu beiträgt das „Valley of Death“ bis zur ersten Markteinführung zu überbrücken. Zusätzlich zu dieser monetären Förderung existiert noch eine Reihe von „soft measures“, die einschlägiges Knowhow zum Thema Unternehmensgründung zur Verfügung stellt. 
Wichtig zu betonen ist es, dass die Sicherstellung der Finanzierung der Unternehmensgründung sowie der ersten Phase des Unternehmensaufbaus via PreSeed und Seedfinancing nur eine „Initialzündung“ darstellt. Gerade im Hochtechnologiebereich (hier wiederum insbesondere in den Life Sciences) sind der Unternehmensaufbau sowie die Skalierung der Geschäfts- bzw. Produktidee oft nur sehr langfristig möglich und mit sehr hohen Kosten verbunden. Vielfach müssen auch bereits während der Markteinführungsphase aufgrund der entsprechenden Rückkoppelung Nachbesserungen und u.U. auch grundsätzlichere Änderungen der ursprünglichen Geschäftsidee vorgenommen werden („pivoting“). In Anschluss an die Förderung durch die JITU-Programme sind dazu zusätzliche externe Finanzierungsquellen in Form von Risikokapital vielfach unumgänglich. Angesichts der derzeitigen Lage des privaten Risikokapitalmarkts in Österreich (nur sehr geringe Volumina aus privatem Fundraising für Frühphasen VC) sind daher die risikokapitalbezogenen Instrumente (aws-Gründerfonds, aws-EIF-Business Angel Fonds, aws-Venture Capital Initiative sowie als "Matchmaker" das 12-Programm) ebenfalls als integraler Bestandteil der technologieorientierten Gründungsförderung, gleichsam komplementär zum JITU-Programm, zu betrachten. Gemeinsam mit der projektbezogenen F\&E-Förderung der FFG sowie ausgewählten zusätzlichen FFG-Programmen (hier vor allem AplusB, Markt.Start, Startup-Förderung oder Projekt-Start) bilden diese Programme mit ihren unterschiedlichen Instrumenten somit ein „Ökosystem“ zur Etablierung einer dynamischen Startup-Szene in Österreich in der sich die in Kapitel 1.2 skizzierten positiven externe Effekte tatsächlich entfalten können.

\section{Abbildung 4: Förderinstrumente entlang des Unternehmenszyklus}

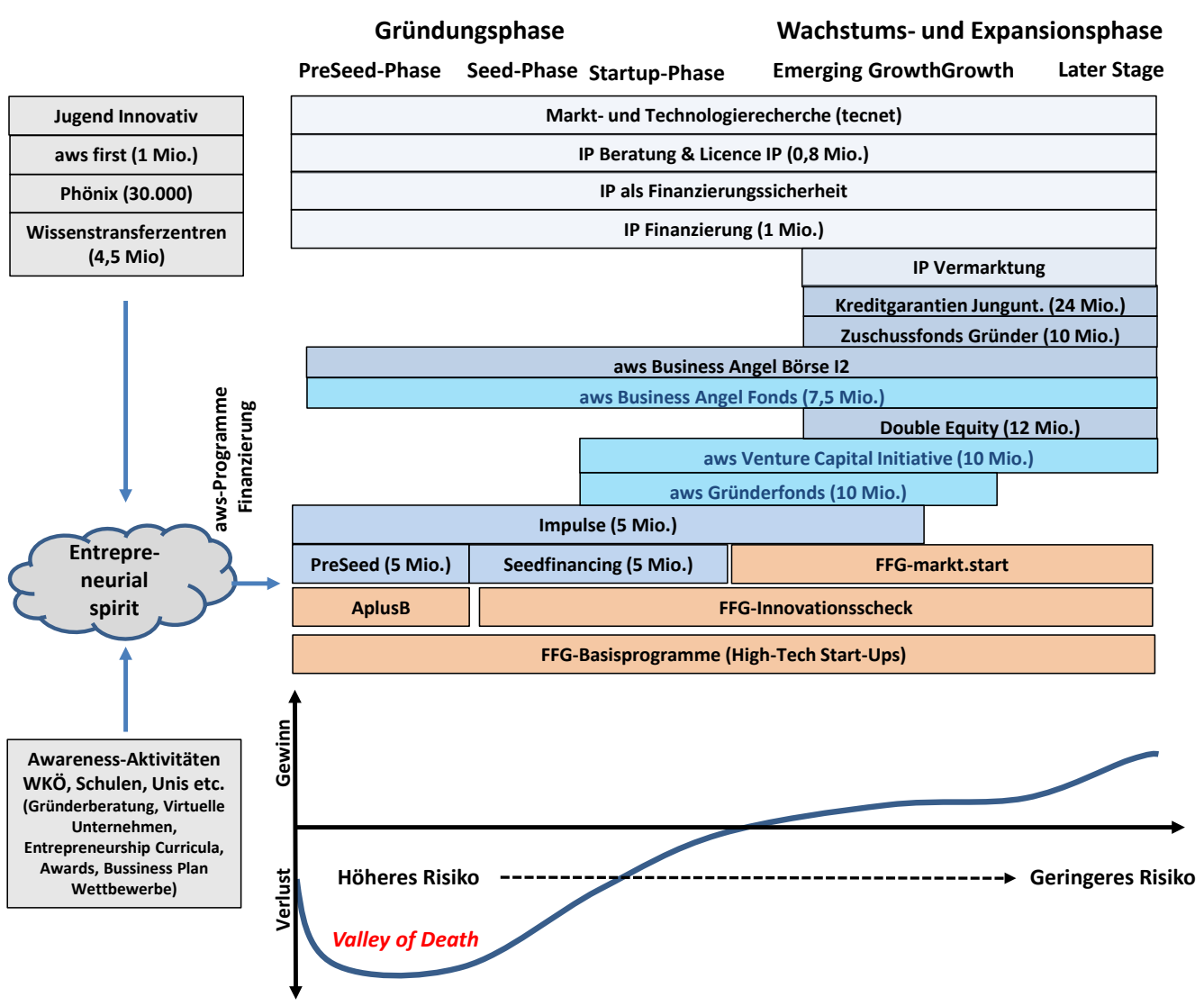

Quelle: eigene Darstellung; Anm.: Bei den Angaben zu den Fördermitteln handelt es sich um grobe Richtwerte.

Hervorzuheben ist daher, dass sich diese unterschiedlichen Instrumente gegenseitig ergänzen bzw. komplettieren. Zum Beispiel stellt die Finanzierung des Unternehmensaufbaus durch Seedfinancing sicher, die für die Durchführung von FFG-geförderten F\&E-Projekten notwendigen Eigenmitteln nachgewiesen werden können. Auch die Kombinationsmöglichkeiten der unterschiedlichen aws-Programme selbst, stellen 
diesen „systemischen Charakter“ der (technologieorientierten) Gründungsförderung sicher (vgl. Abbildung 5). Die JITU-Programme PreSeed, Seedfinancing und MaZ bilden gemeinsam mit i2 sowie dem Business Angel Fonds einen „Programmcluster“, der dennoch mit anderen relevanten Programmschienen verknüpft werden kann.

Abbildung 5:Programmkombinationsmöglichkeiten von Förder-, Beratungs- und Awarenessmaßnahmen der aws

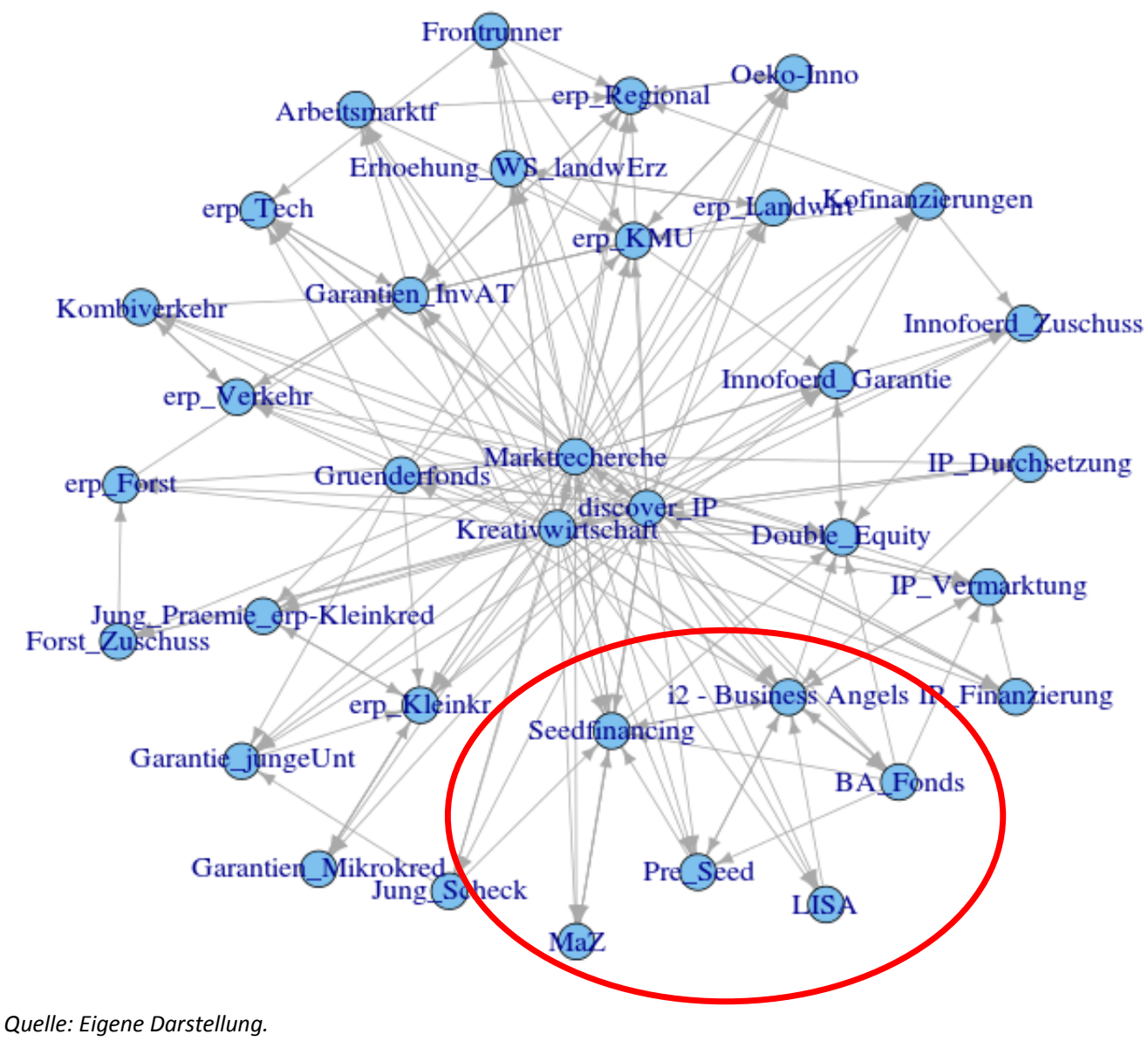

Durch die Kombinierbarkeit der unterschiedlichsten Programme zur Gründerförderung entsteht eine konsekutive "Förderkette“, die den Prozess des Unternehmensaufbaus von der (Vor-)Gründungsphase“ bis zur Wachstumsphase begleitet. In Abbildung 6 ist ein Beispiel für eine derartige „Förderkette“ dargestellt, wobei hier auf die Daten des Wiener AplusB-Zentrums INiTS zurückgegriffen wird. Es zeigt sich, dass vielfach bereits während der INiTS-Betreuungsphase (bzw. in Ausnahmefällen auch schon davor) andere Förderquellen erschlossen werden können. Hinsichtlich der Kombination mit den JITU-Programmen PreSeed und Seedfinancing ergibt sich näherungsweise eine Entsprechung zur „idealtypischen“ Konzeption der einzelnen Programme. Die Kombination INiTS und PreSeed findet sich gehäuft in den frühen Monaten der INiTS-Betreuung, während Seedfinancing dann gegen Ende der AplusB-Betreuungszeit auftritt. Dieses Bild bestätigt somit, die bereits in der Zwischenevaluierung der aws-Technologieprogramme im Jahr 2011 festgestellte Beobachtung (Gassler et al., 2011), dass die AplusB-Zentren eine wichtige „Pipeline-Funktion“ für die JITU-Programme aufweisen. 
Abbildung 6: Förderkette für innovative Gründungen am Beispiel der Kombination von INiTS-betreuten Gründungen mit anderen Förderinstrumenten

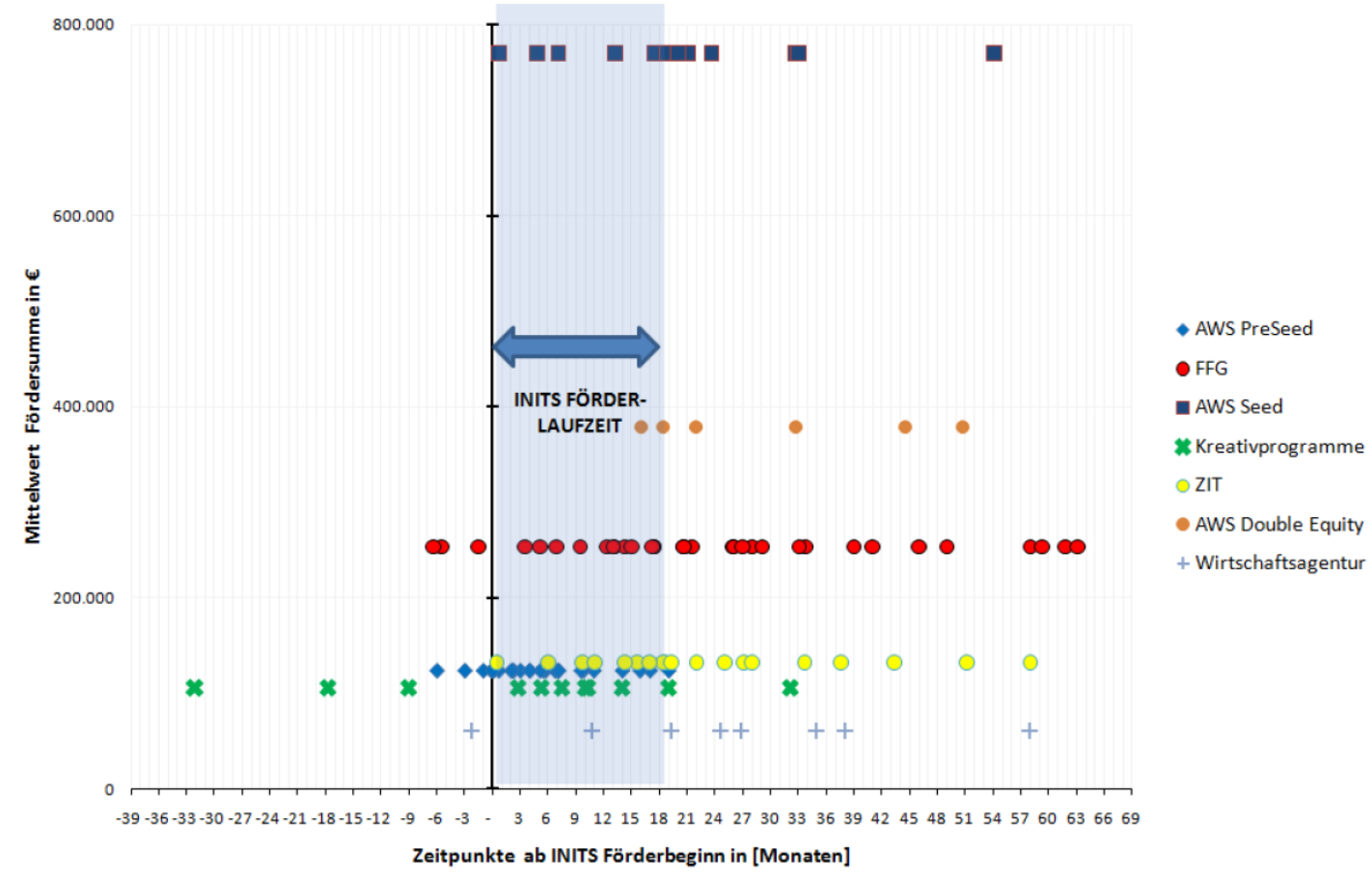

Quelle: INiTS.

In Abbildung 7 ist die zeitliche Entwicklung der Auszahlungen ${ }^{2}$ für technologieorientierte, innovative Gründungen durch das JITU-Programm der aws sowie durch die einschlägigen Instrumente der FFG dargestellt. Seit 2008 sind die ausbezahlten Förderungen deutlich angestiegen und zwar von gesamt 7,4 Mio. im Jahr 2008 auf 21,6 Mio. EUR im Jahr 2013. Insbesondere die Mittel des JITU-Programms der aws haben sich stark erhöht. Der Anstieg von 4,7 Mio. (2008) auf 13,9 Mio. EUR (2013) bedeutet annähernd eine Verdreifachung der Mittelauszahlung innerhalb des hier betrachteten Zeitraums.

\footnotetext{
2 Hierbei sei angemerkt, dass Auszahlungen jene Beträge darstellen die während eines Jahres ausbezahlt werden. Davon zu unterscheiden sind während eines Jahres zugesagte Fördermittel. Letztere müssen nicht zwingend im Jahr der Zusage auch ausbezahlt werden. Daher unterscheiden sich in der Regel die Auszahlungen von den Förderzusagen.
} 


\section{Abbildung 7: Auszahlungen (Förderungen) an Hightech-Gründungen in Österreich}

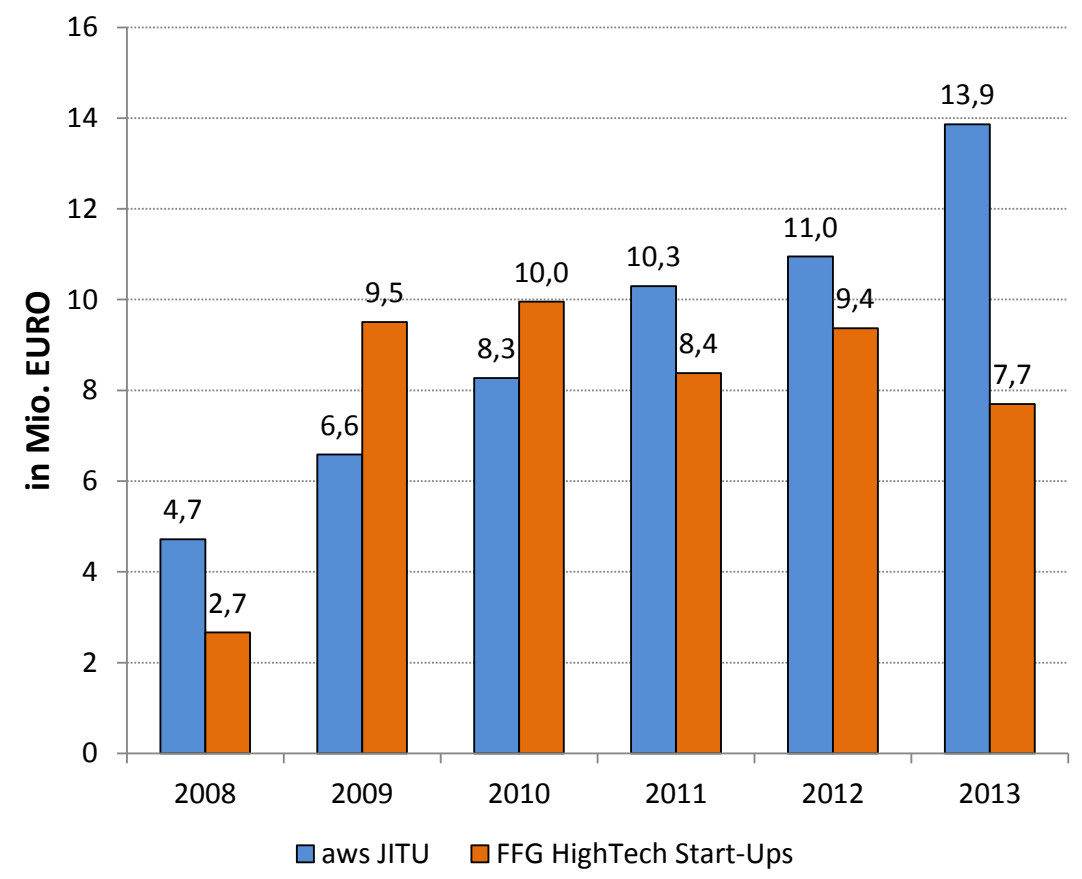

Quelle: aws, FFG Jahresberichte.

Seit einigen Jahren wird von der einschlägigen Literatur eine systemische Betrachtung der Gründungsszene eingefordert (Schramm, 2006, OECD, 2014, Acs et al, 2014): „Remember that entrepreneurial activity does not exist in a vacuum “ (Lerner. 2010, 261). Unter einem so genannten „entrepreneurial ecosystem“ wird die Gesamtheit der Individuen und Institutionen sowie deren Interaktionen verstanden, die in verschiedenster Art und Weise eine Rolle bezüglich des Ausmaß und Struktur der Gründungsaktivitäten spielen (siehe Abbildung 8). Darunter fallen natürlich die (potentiellen) Gründer selbst, Inkubatoren wie z.B. Universitäten und sonstige Forschungseinrichtungen, Förderinstitutionen, regulative bzw. administrative Rahmenbedingungen, Business Angels und institutionelle Risikokapitalgeber, spezialisierte Dienstleister (z.B. Steuerberater oder Juristen mit einschlägiger Spezialisierung) etc. Hervorgehoben wird, dass vor allem das Ausmaß und die Qualität der Interaktionen zwischen diesen Akteuren bzw. Institutionen die Qualität und Dynamik eines derartigen Ökosystems bestimmt. Viele dieser Interaktionen erfolgen dabei über (vielfach informelle) personelle Netzwerke, wodurch so genanntes „Sozialkapital“ generiert wird. Im günstigsten Fall können sich dann die bereits beschriebenen kumulativen und zirkulären Effekte voll entfalten.

Für die Ausgestaltung der Politik hat diese systemische Betrachtung zur Folge, dass die einzelnen Politikelemente (Gründungsförderung, Risikokapitalmarktpolitik, Rahmenbedingungen etc.) optimal aufeinander abgestimmt sein sollten. Fehlt eine Komponente (z.B. Risikokapitalmarkt bzw. -politik) hat das wiederum negative Effekte auf die anderen Komponenten. 


\section{Abbildung 8: Schematische Darstellung des Start-Up Ökosystems}

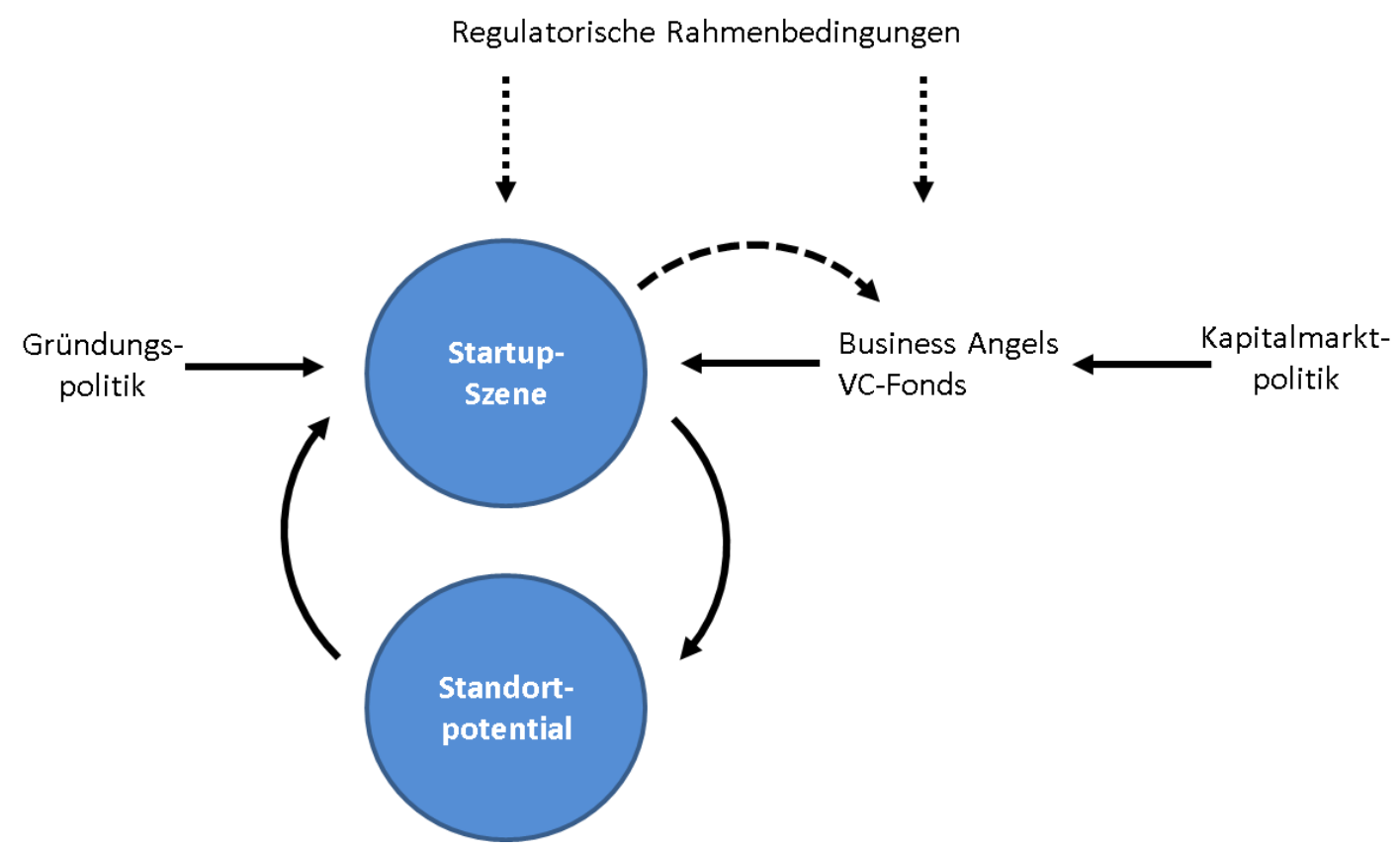

Quelle: eigene Darstellung

\subsection{Exkurs: Finanzierung von High-Tech-Gründungen in Österreich durch Risikokapital}

Nachdem in Kapitel 1.3 betont wurde, dass (privates) Risikokapital für den langfristigen Erfolg bzw. die Skalierung der Geschäftsidee (und somit für die Erzielung entsprechend hoher volkswirtschaftlicher Effekte) von großer Bedeutung ist, soll im Folgenden in Form eines kurzen Exkurses ein Überblick über den Status Quo des Frühphasen-Risikokapitalmarkts gegeben werden.

Kontinentaleuropa ist im Vergleich mit den USA und Großbritannien bezüglich des Entwicklungsniveaus seiner Risikokapitalmärkte deutlich im Rückstand. Während die USA bereits seit Jahrzehnten einen „reifen“ privaten Risikokapitalmarkt aufweisen, ist die Branche in den meisten EU-Ländern noch sehr "jung“ bzw. befindet sich überhaupt erst noch in einem „infant“ Stadium. Anhand der Tabelle 1 lässt sich erkennen, dass USA und UK schon im Jahr 1989 ein relativ hohes Ausmaß an Risikokapital in der Frühphase aufwiesen. Damals war der österreichische Risikokapitalmarkt (Frühphasen VC in \% des BIP) annähernd auf dem Niveau von den Niederlanden, Dänemarks, Finnlands, Irlands oder Frankreichs. Während diese Länder jedoch im Zuge des „New Economy-Hypes“ eine dynamische Entwicklung ihrer Risikokapitalmärkte durchmachten, kam es in Österreich zu keinem vergleichbaren "Take off“ und Österreich blieb deutlich hinter der Entwicklung in diesen Ländern zurück. Die beiden rechten Spalten der Tabelle 1 zeigen dann die Folgen der jüngsten Finanz- und Wirtschaftskrise auf den Risikokapitalmarkt in Europa. Mit wenigen Ausnahmen zeigt sich ein (teilweise sehr deutlicher) Rückgang der Frühphasen VC-Mittel in Relation zum BIP. Neben dem Rückgang der Risikokapitalmittel generell (durch geringere Volumina im Fundraising) wurden auch die VCFonds nach der Finanz- und Wirtschaftskrise deutlich risikoaverser und verlagerten ihre Tätigkeit verstärkt in spätere Phasen. ${ }^{3}$

\footnotetext{
${ }^{3}$ Siehe Wilson, K. and F. Silva (2013).
} 
Tabelle 1: Frühphasen VC-Mittel in \% des BIP, 1989-2012

\begin{tabular}{lrrrr} 
& 1989 & 1999 & 2007 & 2012 \\
\hline Österreich & $0.006 \%$ & $0.007 \%$ & $0.013 \%$ & $0.010 \%$ \\
Belgien & $0.015 \%$ & $0.093 \%$ & $0.024 \%$ & $0.016 \%$ \\
Dänemark & $0.009 \%$ & $0.019 \%$ & $0.058 \%$ & $0.021 \%$ \\
Finnland & $0.003 \%$ & $0.057 \%$ & $0.035 \%$ & $0.034 \%$ \\
Frankreich & $0.009 \%$ & $0.039 \%$ & $0.019 \%$ & $0.013 \%$ \\
Deutschland & $0.004 \%$ & $0.051 \%$ & $0.016 \%$ & $0.013 \%$ \\
Irland & $0.002 \%$ & $0.048 \%$ & $0.016 \%$ & $0.039 \%$ \\
Italien & $0.002 \%$ & $0.014 \%$ & $0.003 \%$ & $0.004 \%$ \\
Niederlande & $0.006 \%$ & $0.096 \%$ & $0.024 \%$ & $0.014 \%$ \\
Portugal & $0.004 \%$ & $0.008 \%$ & $0.008 \%$ & $0.009 \%$ \\
Spanien & $0.009 \%$ & $0.018 \%$ & $0.010 \%$ & $0.007 \%$ \\
Schweden & $0.004 \%$ & $0.113 \%$ & $0.073 \%$ & $0.025 \%$ \\
Vereinigtes Königreich & $0.023 \%$ & $0.020 \%$ & $0.025 \%$ & $0.025 \%$ \\
USA & $0.027 \%$ & $0.056 \%$ & $0.042 \%$ & $0.051 \%$ \\
\hline
\end{tabular}

Quelle: EIB (2001), EVCA, NVCA, Weltbank-eigene Berechnungen.

In Tabelle 1 ist die Anzahl der Unternehmen, welche eine VC-Finanzierung erhalten haben, normiert je Mio. Einwohner dargestellt. Zu beachten ist, dass es - nicht zuletzt aufgrund des geringen Aktivitätsvolumens in etlichen Ländern eine starke Volatilität in der zeitlichen Entwicklung gibt. Daher sind hier die entsprechenden Werte für den Zeitraum der Jahre 2007 bis 2012 dargestellt, wobei die Länder nach ihrer Medianausprägung gereiht wurden. An vorderster Stelle finden sich die nordischen Länder, während Österreich etwa im Mittelfeld liegt. Bemerkenswert ist, dass die USA mit einem relativ geringen normierten Aktivitätsniveau sogar noch hinter Österreich. Zu betonen ist, dass in den USA der Risikokapitalmarkt sehr stark auf einige wenige High-Tech-Regionen (allein voran Kalifornien, Massachusetts, Texas etc.) konzentriert ist und in vielen anderen Bundesstaaten ebenfalls nur gering ausgeprägt ist. Diese räumliche Konzentration des Risikokapitalmarkts ist auf die ebenfalls sehr hohe räumliche Konzentration der HighTech Startup Szene in den USA zurück zu führen. Es sind das jene Regionen in denen ein funktionierendes Ökosystem zwischen technologieorientierter Gründerszene und dem Risikokapitalmarkt zu den entsprechenden externen Effekten mit den daraus resultierenden zirkulären und kumulativen Prozessen, wie sie in Kapitel 1.2 beschrieben worden sind, ausgeprägt ist.

Der Vergleich von Abbildung 9 mit Abbildung 10 zeigt das eigentlich Problem des europäischen (und gleichermaßen des österreichischen) Risikokapitalmarkts auf. Es sind nämlich die geringen verfügbaren VCMittel und weniger die reine Anzahl der Investitionsfälle. Die normierten Investitionssummen je Mio. Einwohner zeigen nämlich eine deutlich andere Rangreihung der Länder. Diesbezüglich nimmt die USA eine Spitzenstellung unter den hier betrachteten Ländern ein, während Österreich nunmehr in das hintere Mittelfeld zurückfällt. 
Abbildung 9: Investierte VC-Unternehmen pro EW, 2007-2012

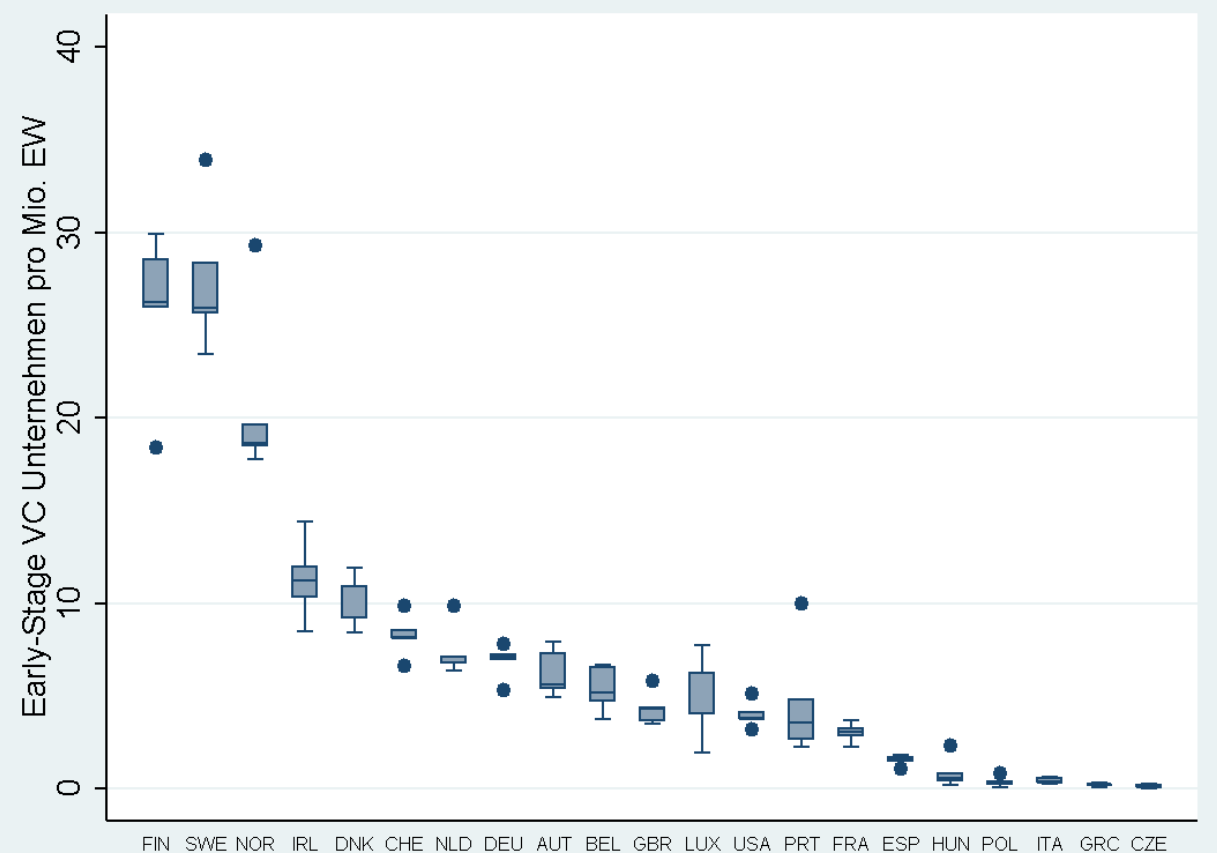

Quelle: EVCA, NVCA, Weltbank - eigene Berechnungen.

Abbildung 10: Investiertes VC-Volumen pro EW, 2007-2012

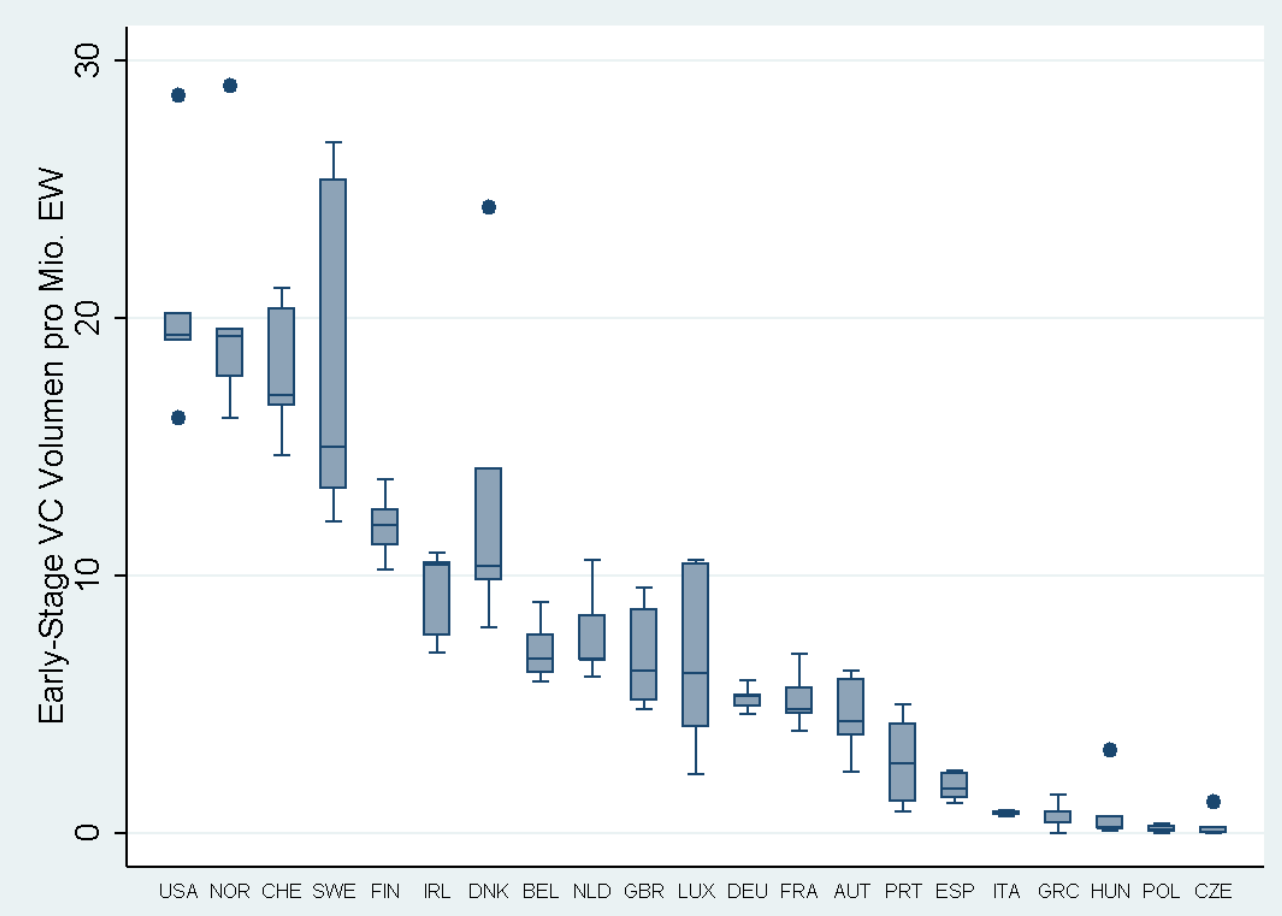

Quelle: EVCA, NVCA, Weltbank - eigene Berechnungen. 


\subsection{Exkurs: Zum Stellenwert von Business Angels im Gründungsgeschehen Österreichs}

Nachdem es auch Aufgabe dieser Evaluierung ist auch das 12 Programm der aws einzuschätzen, soll im Folgenden anhand eines kurzen Exkurses ein Überblick über die Business Angel Szene in Österreich und deren jüngste Entwicklung gegeben werden. Neben quantitativen Daten werden dabei auch einzelne Ergebnisse diverser Interviews und (informelle) Gespräche mit Business Angels (z.B. im Zuge von Investorenmeetings und sonstiger Veranstaltungen) als Grundlage genommen.

Neben den „klassischen“ institutionellen VC Fonds spielen zunehmend auch Privatpersonen in Form von Business Angels (teilweise auch in Form von Netzwerken mehrerer Privatpersonen als so genannte Business Angels Groups) eine wichtige Rolle für die Finanzierung von technologieorientierten, innovativen Gründungen mit entsprechendem Wachstumspotential. In jüngster Zeit wird auch von sogenannte, Super Angels', deren Investitionsvolumina pro Beteiligung deutlich über den in der Literatur üblicherweise genannten Beträgen von ca. 25-500k EUR/Beteiligung zum Tragen kommen gesprochen.

Die Bedeutung von Business Angels trifft insbesondere auf die Frühphasen des Unternehmensaufbaus zu, wo einerseits der Kapitalbedarf zwar noch vergleichsweise gering ist, aber anderseits die Investitionsbereitschaft von institutionellen VC-Fonds noch nicht gegeben ist. In dieser Phase ergänzen dann die Business Angels die so genannten „3Fs“ (Family, Friends and Fools) als externe Kapitalgeber.

Die jüngste Literatur betont die zunehmende Rolle von Business Angels, da sich im Zuge der Finanz- und Wirtschaftskrise viele institutionelle Fonds aus der Frühphasenfinanzierung zurückgezogen haben: „the majority of venture capital firms have moved to later stage investment leaving the seed and early stage market to "informal' investors." (OECD, 2014, 5). Business Angels erfüllen aber nicht ausschließlich eine Finanzierungsfunktion. Neben der Bereitstellung von Risikokapital stellen sie nämlich den Gründungen vielfach auch noch eine ganze Reihe von intangiblen Ressourcen zur Verfügung. Meist weisen Business Angels einen reichen unternehmerischen Erfahrungsschatz (z.B. als Vorstand / Geschäftsführer eines Unternehmens oder haben selbst einen Hintergrund als selbständiger Unternehmer). Dadurch können sie der Gründung wertvolles Knowhow zur Verfügung stellen. Zudem bringen sie häufig Kontakte und Netzwerkbeziehungen (Sozialkapital) in das neue Unternehmen ein: „There is an increasing recognition ... that business angels play a very important and increasing role in the ecosystem as they not only provide funding (supply side) but also experience, credibility, contacts and connections that improve the flow of high-quality firms available to the VC sector (demand side)." (Duruflé, 2010). Damit fungieren Business Angels auch als Mentoren und „door opener“ für Gründungen. Im günstigsten Fall weisen Business Angels als ,serial entrepreneur' selbst als Startup-Gründer erworbenes einschlägiges Knowhow bezüglich des Unternehmensaufbaus auf, das sie dann direkt in das neu gegründete Unternehmen einbringen können.

Die wachsende Bedeutung von Business Angels zeigt sich empirisch an der starken Zunahme der Risikokapitalinvestitionen dieser Gruppe in etlichen Ländern (siehe Abbildung 11). Gerade in Österreich war das Wachstum zwischen den Jahren 2011 und 2012 mit einer Steigerung um 116 \% besonders stark ausgeprägt (jedoch von einem Niveau von unter 10 Mio. Euro ausgehend). 
Abbildung 11: Wachstum der Risikokapitalinvestitionen von Business Angels, 2011 auf 2012

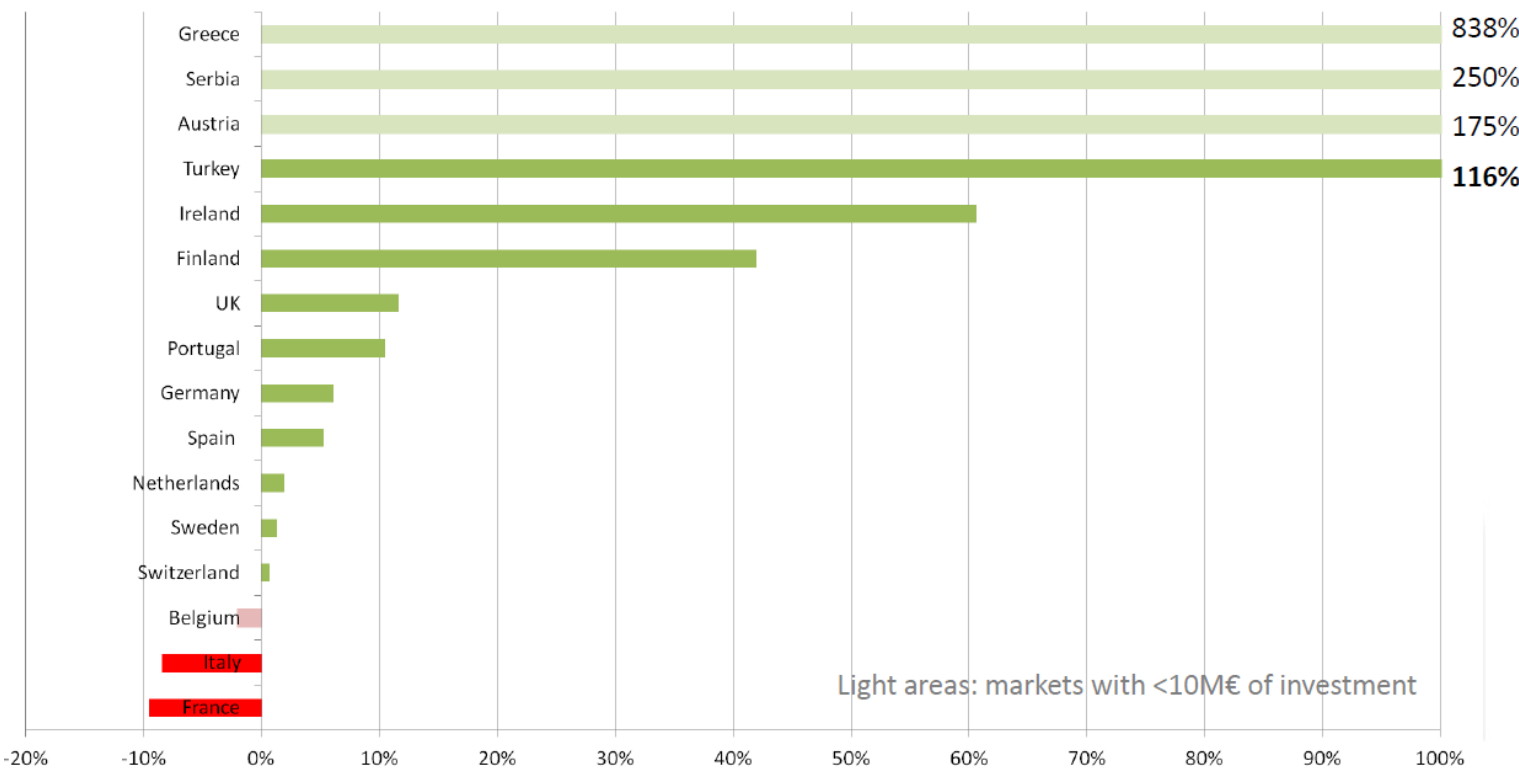

Quelle: EBAN, European Angel Investment Overview 2012.

Dieses starke Wachstum in Österreich geht allerdings noch von einem sehr geringen Niveau aus (Abbildung 12). Im Jahr 2012 betrugen die gesamten Investitionen von Business Angels in Österreich erst lediglich 2,6 Mio. EUR und lagen somit teilweise sehr deutlich unter dem Niveau von Ländern mit annähernd vergleichbarer Größe (wie z.B. Belgien, Irland, Schweden oder den Niederlanden).

Abbildung 12: Business Angel Investitionen 2012

BA Investitionen (2012)

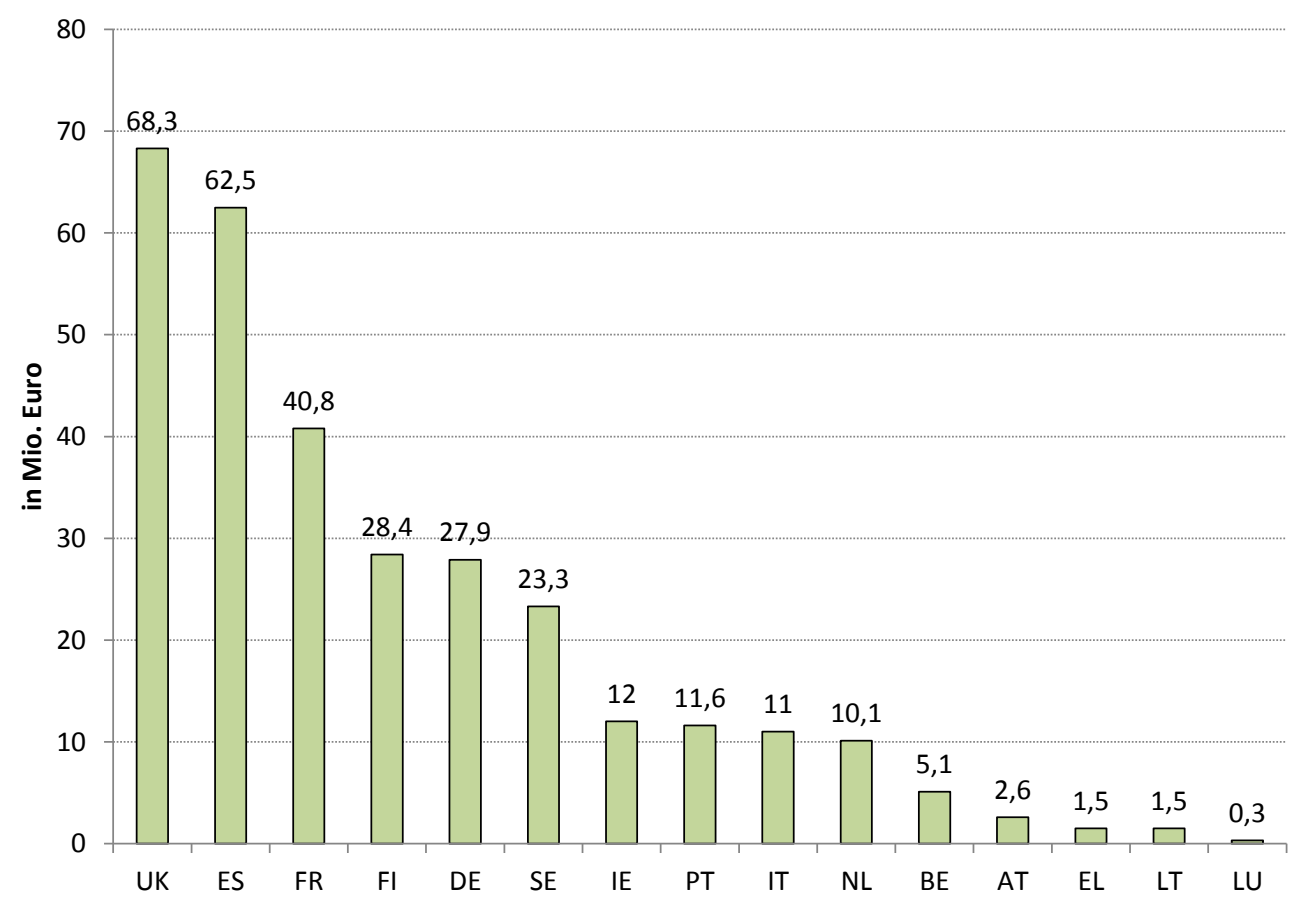

Quelle: EBAN (2013).

Die Wirtschaftspolitik vieler OECD-Länder (darunter auch Österreich) knüpft an diese steigende Bedeutung von Business Angels für die Gründungsszene explizit an und hat darauf mit dem Design spezifisch zugeschnittener Programme und Instrumente reagiert. Typische Beispiele für einschlägige Instrumente sind 
steuerliche Erleichterungen bzw. Anreize für Risikokapitalinvestitionen von Business Angels (v.a. in Großbritannien), Co-Investment Funds (u.a. mit dem EIF, dem European Investment Fund), die das private Investment hebeln, sowie unterschiedliche Matching Services (ähnlich wie i2 der aws) und Business Angel Networks etc.

Die Zunahme der Rolle von Business Angels in Österreich lässt sich auch an der wachsenden Zahl von Business Angels zeigen. In Einzelinterviews mit Insidern bzw. Stakeholdern wurde die Zahl der aktiven Business Angels in Österreich auf derzeit ca. 200 geschätzt. Allerdings wird angemerkt, dass allerdings von dieser Gruppe lediglich vier bis fünf Investoren ihre Rolle professionell, gleichsam als „Hauptberuf“ definieren. 


\section{Beschreibung der Programme}

Im Folgenden sollen - ausgehend von den Programmrichtlinien - die Ziele, sowie die konkrete Ausgestaltung der JITU-Programme bzw. Instrumenteneinsatz sowie die fördertechnische Ausgestaltung beschrieben werden. In den „Richtlinien zur Förderung von Gründungen und Aufbau junger innovativer technologieorientierter Unternehmen (JITU-Richtlinien) “4 ${ }^{4}$ (Fassung Juni 2014) wird als Zielsetzung der Richtlinien folgendes erörtert:

„Die Richtlinien sollen die besonderen Anforderungen an die Förderung von Gründungen und Aufbau junger innovativer technologieorientierter Unternehmen erfüllen. Die Gründung von jungen technologieorientierten Unternehmen soll erleichtert und ihre Überlebensrate gesteigert werden. Dadurch soll sich der Anteil dieser Unternehmen an der Gesamtpopulation der österreichischen KMU dauerhaft erhöhen. Dies soll durch eine ordnungsgemäße und transparente Vergabe dieser Förderung innerhalb des Beihilferechts der EU ermöglicht werden." [Hervorhebungen durch die Autoren]

Weiters ist besonderes „Augenmerk ist auf die Förderung von Projekte zu legen welche eine Hebelwirkung in Richtung Forschung, Entwicklung und Innovation aufweisen“. Eine generellere Zielsetzung der Richtlinie ist es ein geeignetes Instrumentarium zur Unterstützung von potentiellen Gründern und zur Förderung junger kleiner innovativer technologieorientierter Unternehmen zu schaffen. Dieses konkrete Instrumentarium findet sich im „Programm zur Förderung von Gründungen und Aufbau junger innovativer technologieorientierter Unternehmen “" ${ }^{5}$ (Fassung Juni 2014) des Bundesministeriums für Wissenschaft, Forschung und Wirtschaft. Darin werden die bereits vor der Richtlinie bestehenden Programme "PreSeed“, „Seedfinancing“ und das mit Inkrafttreten der JITU-Richtlinie (Fassung 2007) ins Leben gerufene Programm „Management auf Zeit“ genauer beschrieben. Die Laufzeit der 3 Programmmodule wird in der Programmdokumentation in der Fassung Juni 2014 mit 1. Juni 2013 bis 31 . Dezember 2014 festgeschrieben. Eine genauere Diskussion der Budgetierungsperioden erfolgt in Abschnitt 4.1.

\subsection{PreSeed}

Die Zielsetzung des PreSeed Förderprogramms (Modul 1 des JITU Programmdokuments) ist die „nachhaltige Gründung von wettbewerbsfähigen, wirtschaftlich selbständigen, kleinen Hochtechnologieunternehmen mit ausgeprägten Forschungs- und Entwicklungsaktivitäten und die Überleitung von Forschungsergebnissen in wettbewerbsfähige Produkte, Verfahren oder Dienstleistungen“. Weiters soll das Risiko von Unternehmensgründungen im Hochtechnologiebereich gesenkt und die Zahl der Unternehmensgründungen nachhaltig erhöht werden. Mit LISA-PreSeed existiert das Programm bereits seit 2003 für den Life Science Bereich und wurde im Jahr 2005 auch auf den Programmschwerpunkt IKT \& Physical Sciences ausgeweitet. Die maximale Förderhöhe von 100.000 Euro (im Rahmen der „de-minimis“Regelung des EU-Wettbewerbsrechts) wurde mit dem 4. Quartal 2009 auf 200.000 Euro erhöht, um der Komplexität und dem hohen Kostenaufwand, die mit technologieintensiven Gründungen verbunden sind, Rechnung zu tragen. Die Auszahlung der Fördermittel erfolgt gemäß einem vertraglich fixierten Meilensteinkonzept. Die Laufzeit beträgt typischerweise ein Jahr, laut Programmdokument sollte das Vorhaben innert 2 Jahren abgeschlossen werden.

Mittels des Programm PreSeed soll die Finanzierungslücke in der Gründungsfinanzierung welche sich durch asymmetrische Informationen und zu hohe Transaktionskosten am privaten Kapitalmarkt ergibt adressiert

\footnotetext{
${ }^{4}$ Siehe https://www.awsg.at/Content.Node/richtlinie/JITU.pdf. Diese Richtlinien basieren auf dem Bundesgesetz zur Förderung der Forschung und Technologieentwicklung (Forschungs- und Technologieförderungsgesetz FTFG, BGBI. Nr. 434/1982.

${ }^{5}$ Siehe https://www.awsg.at/Content.Node/media/files/JITU.pdf.
} 
werden. Innerhalb des JITU-Daches erfüllt PreSeed auch die Funktion, dem Modul 2 Seedfinancing entsprechend attraktive gegründete Unternehmen zu liefern. Durch eine frühzeitige Bewertung (Überprüfung auf Machbarkeit und Kommerzialisierungspotential) und monetäre Unterstützung soll das Projekt hin zu einer marktfähigen Geschäftsidee begleitet werden.

Förderbare Kosten sind Personalkosten von mit dem Vorhaben beschäftigten Forschern und Technikern, Projektbezogene Sachkosten (z.B. Laborgeräte) im Ausmaß des Wertverlustes (Abschreibung), Kosten für Patente und Schutzrechte, Beratung durch externe ExpertInnen, Konzept- und Studienkosten, projektrelevante Expertisen zu Entwicklung, Produktdesign etc. und Reise- und Ausbildungskosten.

Der Förderwerber muss über die entsprechenden technischen und wirtschaftlichen Voraussetzungen für eine erfolgreiche Unternehmensgründung verfügen und es darf in den letzten 5 Jahren kein EntziehungsDisziplinar-, Konkurs- oder Ausgleichsverfahren gegen inn verhängt worden sein. Zudem darf er noch kein Unternehmen zu dem Projektgegenstand gegründet haben und muss das geplante Unternehmen in Österreich gründen.

Die wichtigsten inhaltlichen Beurteilungskriterien des geplanten Projektes sind

- Technologiesprung

- Patentierbarkeit bzw. anderweitige Absicherung des geistigen Eigentums

- Kommerzialisierbarkeit (skalierbare Absetzmöglichkeiten)

- Hochqualifizierte, engagierte, risikobereite und gründungsorientierte Förderwerber.

Es werden somit keine inkrementellen Weiterentwicklungen gefördert.

Die Förderung erfolgt über nicht rückzahlbare Zuschüsse in Höhe von bis zu $100 \%$ der förderbaren Projektkosten, wobei die Auszahlung in erfolgsabhängigen Tranchen anhand eines Meilensteinkonzepts erfolgt. PreSeed Förderungen sind mit den Programmen Seedfinancing und i2 - Business Angel der aws kombinierbar.

\subsection{Seedfinancing}

Seedfinancing (Modul $2 \mathrm{im} \mathrm{JITU-Programmdokument)} \mathrm{wurde} \mathrm{bereits} \mathrm{im} \mathrm{Jahr} 1989$ etabliert (damals im Rahmen des IFT-Fonds, der dann später in die aws überging) und weist somit eine außerordentlich lange Kontinuität auf. Seit 2004 wird das Programm - zunächst gemeinsam vom damaligen BMWA (heute BMWFW) und dem BMVIT im Rahmen ihrer jeweiligen Schwerpunktfelder beauftragt - und von der aws operativ abgewickelt. Seit 2010 ist das heutige BMWFW der alleinige Prinzipal des Programms. Als das von den zur Verfügung stehenden Mitteln her größtes Kernmodul innerhalb der „Richtlinien zur Förderung von Gründung und Aufbau junger, innovativer, technologieorientierter Unternehmen“ (JITU-Richtlinien) kann es als das zentrale Programm zur Dynamisierung der High-Tech-Gründungskultur in Österreich bezeichnet werden.

Zielsetzung laut Programmdokument ist „die Erhöhung der Anzahl von technologieorientierten Unternehmensneugründungen sowie die Unterstützung des Aufbaus von Unternehmen zur wirtschaftlichen Nutzung innovativer und technologisch avancierter Produktideen, Verfahren oder Dienstleistungen mit überdurchschnittlichem Marktpotential und Wachstumschancen durch die Bereitstellung einer SeedFinanzierung und einer begleitenden Beratung". Zudem bietet Seedfinancing Unterstützung bei der Ausweitung der Kapitalbasis (vor allem Risikokapital).

Die wirtschaftspolitische Motivation hinter den Zielsetzungen des Seedfinancing Moduls stehen letztlich im Einklang des Lissabon-Prozesses bzw. Horizon 2020 und können wie folgt zusammengefasst werden: 
- Verbesserung der österreichischen Wirtschaftsstruktur durch die Fokussierung auf dynamische, technologieorientierte Branchen sowie die Schaffung dauerhafter, hochqualitativer Arbeitsplätze

- Erhöhung der Anzahl von technologieorientierten Unternehmensneugründungen

- Nachhaltige Unterstützung des Aufbaus von Unternehmen zur wirtschaftlichen Nutzung innovativer und technologisch avancierter Produktideen, Verfahren oder Dienstleistungen mit überdurchschnittlichem Marktpotential und Wachstumschancen durch die Bereitstellung einer Seed-Finanzierung und einer projektbegleitenden Beratung

- Förderung von dynamischem und qualitativem Wachstum von innovativen Unternehmen

- Unterstützung bei der Ausweitung der Kapitalbasis (insbesondere für Risikokapital).

Beim Fördernehmer muss es sich um ein kleines innovatives Unternehmen (mit Sitz in Österreich) handeln, das zum Zeitpunkt der Fördergewährung weniger als sechs Jahre bestanden hat. Ein innovatives Unternehmen liegt laut Programmdokument vor, wenn die F\&E Aufwendungen des Begünstigtem in einem der drei Jahre vor Gewährung der Beihilfe mind. 15\% der beglaubigten Betriebsausgaben ausgemacht haben. Seedfinancing darf bis zur maximalen Förderhöhe nur einmal innerhalb des Zeitraums in dem das Unternehmen als jung eingestuft wird bezogen werden. Zudem dürfen in den ersten drei Jahren nach Bewilligung nebst Seedfinancing nur F\&E\&I-Beihilfen und Risikokapitalbeihilfen bezogen werden. Seedfinancing ist diesbezüglich mit den weiteren aws Programmen Management auf Zeit (MaZ), Double Equity, i2 - Business Angels, Marktrecherche und discover.IP kombinierbar.

Die Bewertungskriterien für die Förderfähigkeit entsprechen jenen des PreSeed Moduls, mit dem Zusatz, dass die Wahrscheinlichkeit der Weiterfinanzierung über private Equity/Venture Capital nach der Seedphase besteht. Die genauen Förderkriterien sind in einem gesonderten Bewertungshandbuch festgelegt. Gefördert werden neben den förderbaren Kosten des PreSeed Moduls auch Betriebsmittel und Markterschließungskosten. Die maximale Förderhöhe beträgt 1 Mio. Euro und Beratungsleistungen im Ausmaß von maximal 450 Stunden. Vor dem Inkrafttreten der JITU-Richtlinie (April 2008) betrug maximal mögliche Förderhöhe 500.000 Euro. Ergänzend zu den rückzahlungspflichtigen Zuschüssen werden Beratungsleistungen der aws im Ausmaß von maximal 450 Stunden bereitgestellt. Diese Leistungen stellen eine nicht-monetäre Förderung dar und werden dem Förderungsgeber in Rechnung gestellt sowie dem Förderungswerber detailliert dargestellt und schriftlich mitgeteilt.

Die Projektlaufzeit wird projektspezifisch vereinbart und ist in der Regel mit 4-7 Jahren begrenzt. Der Förderzeitraum umfasst die Dauer der Projektlaufzeit plus fünf Jahre. Die Förderung erfolgt in Form von bedingt rückzahlbaren Zuschüssen über die Rückzahlung aus Gewinnen bzw. Erlöse über Unternehmensverkauf oder Börsengang. Die Gewinnanteile des Fördergebers werden über den Förderzeitraum berechnet und sind mit max. 50\% des jährlichen Gewinns beschränkt. Der Anteil beträgt projektabhängig zwischen 2 und 15\% der Umsatzerlöse sofern ein positives Ergebnis der gewöhnlichen Geschäftstätigkeit vorliegt. In besonderen Fällen (Liquiditätsengpass) kann ein Zahlungsaufschub gewährt werden.

\subsection{Management auf Zeit - MaZ}

Die Seedphase eines Hochtechnologieunternehmens stellt sehr hohe Anforderungen an die Unternehmer. Da diese meist aus dem wissenschaftlichen Bereich kommen, verfügen die Gründungsteams innerhalb des Seedfinancing oft nicht über die nötigen unternehmerischen Qualitäten, welche schnelles Wachstum (bspw. über professionelles Marketing und raschen Exporteinstieg) begünstigen und die kurze „time to market“ in diesem Segment gewährleisten können.

Die generelle Zielsetzung des dritten Moduls des JITU-Programmdokuments, Management auf Zeit (MaZ), ist es „die Überlebenschance des geförderten Unternehmens durch aktive Hilfestellung und schnelle Problemlösung mittels externer, erfahrener ExpertInnen bei sich abzeichnenden Krisensymptomen (wie z.B. 
Verfehlung von Meilensteinen, Liquiditätsengpässen, etc.) zu erhöhen und dabei die Qualifizierung des geförderten Unternehmens durch den Know-How-Transfer am Ende des ExpertInneneinsatzes zu verbessern".

Förderberechtigt sind demnach Unternehmen welche im Rahmen des Seedfinancing gefördert werden und in deren Unternehmen sich Krisensymptome abzeichnen. Die Projektdauer beträgt in der Regel 6 bis 9 Monate, im Falle von Karenzvertretungen ist eine Dauer von einem Jahr möglich. Förderbare Kosten sind Personal- und Reisekosten von externen Beratern zu einem max. förderfähigen Tagessatz von 1.500 Euro. Die Projektkosten dürfen 100.000 Euro nicht überschreiten. Die Förderung erfolgt über einen nicht rückzahlbaren Zuschuss in Höhe von 50\% der förderbaren Projektkosten (also werden maximal 50.000 Euro gefördert).

\section{4. i2 - Business Angels}

Die Börse für Business Angels (i2) wurde Ende 1996 von der Innovationsagentur GmbH im Auftrag des damaligen BMWA etabliert und fällt nicht unter die geltende JITU-Richtlinie. Österreich war hierbei eines der ersten europäischen Länder, das ein Business Angel Netzwerk gerufen hat. i2 ist Mitglied der EBAN (European Trade Association for Business Angels, Seed Funds, and other Early Stage Market Players) und seit kurzem besteht innerhalb der aws auch der Business Angel Funds, welcher als nationaler Arm des EIF fungiert und bei registrierten Business Angels co-investiert.

Die Plattform i2 - Business Angel verfolgt das Ziel eines Matchings zwischen Ideen (Unternehmen in der Frühphase) und Investoren (Business Angels) um

- dem Marktversagen in der Frühphasenfinanzierung entgegenzuwirken

- $\quad$ zur Schließung ihrer Eigenkapitallücke (equity gap) beizutragen

- Angebot einer offenen und effizienten Plattform für alle Beteiligten (Unternehmen mit Kapital- und Unterstützungsbedarf, Investoren, Netzwerkpartner) bereitzustellen

- möglichst viele Player durch unterschiedliche Awareness- und Vermarktungsmaßnahmen anzusprechen.

Im Kern geht es darum Such- und Transaktionskosten zwischen Unternehmen und Investoren abzubauen. Interessierte Unternehmen können im Rahmen des Programms eine Listung beantragen. Dabei gelten als Kriterien

- $\quad$ eine neuartige Geschäftsidee

- großes Marktpotential

- hohe Wachstumschancen

- klare Alleinstellungsmerkmale

- $\quad$ ein erkennbarer Wettbewerbsvorsprung

- $\quad$ ein qualifiziertes Managementteam.

Somit steht das Programm allen Schwerpunkten offen, adressiert jedoch deutlich Unternehmen aus frühen Phasen. Unternehmen welche auf Basis dieser Kriterien von den Programmverantwortlichen selektiert werden und 390 Euro bezahlen werden für 6 Monate gelistet. Kurzinformationen zum Business Plan der gelisteten Unternehmen werden dann den in der Plattform gelisteten Business Angels für eine Aufwandsentschädigung von 90 Euro im Jahr, weitergeleitet. Finden sich interessierte BA wird der Kontakt über die aws hergestellt. Im Falle einer Vermittlung (von bis ca. 500.000 Euro) wird keine Provision geltend, obwohl ein entsprechender Passus- wider die Praxis - lange Zeit im Programmdokument verankert war. i2 - Business Angels ist mit den aws Programmen Double Equity, Seedfinancing, PreSeed sowie Förderungen aus dem aws Gründerfonds kombinierbar. 


\subsection{Spezifika von High-Tech Gründungen und deren Relevanz für die Ausgestaltung des JITU-Programms}

Für die Ausgestaltung des JITU-Programms sind seine Besonderheiten, die bei Gründungsprojekten bzw. in der Frühphase des Unternehmensaufbaus im Bereich Hochtechnologie, gegeben sind, von hoher Relevanz. Dazu zählen vor allem folgende:

- Viele der Projekte sind sehr langfristig und die Zeit zwischen „Proof of Concept“ und ersten Schritten zur konkreten Markterschließung (und daran anknüpfender Skalierung) kann sich über mehrere Jahre erstrecken. Dies betrifft insbesondere die LISA-Projekte, da sich bei Produkten im Life Science Bereich die Abfolge der klinischen Tests üblicherweise über einen langen Zeitraum erstreckt.

- Die Langfristigkeit, sowie die in den Zielen explizit angestrebte Orientierung auf Hochtechnologie mit besonderem Innovationsgehalt („echte Marktneuheiten), setzen notwendigerweise kritische Finanzierungsmassen voraus. Angesichts der begrenzten Ressourcen an zur Verfügung stehenden Fördermittel bedingt dies letztlich eine hohe Selektivität, d.h. Fokussierung auf einige wenige Projekte (die aber mit entsprechend hohen Fördermittel, d.h. nahe oder an der von den Richtlinien her zulässigen maximalen Förderhöhe) anstatt einer breiteren Streuung über mehr Projekte (die aber dann entsprechend mit jeweils geringeren Fördermittel dotiert werden würden).

- Die geförderten Projekte befinden sich vielfach in einer „Förderkette“ (spezifische Finanzierung von Entwicklungsprojekten z.B. durch die F\&E-Förderung der FFG, andere Programme der aws etc.) und stellen zusätzlich eine Pipeline für weitere Finanzierungsrunden durch potentielle private (und öffentliche) Risikokapitalgeber in Form von Business Angels und/oder institutionellen VC-Fonds dar. Diese Pipeline stellt gleichsam die eine Seite (d.h. die Nachfrageseite nach Risikokapital) am österreichischen Risikokapitalmarkt für Frühphasenfinanzierung dar.

- Der prinzipiell branchenoffene Technologie- bzw. Innovationsfokus (mit Ausnahme des explizit thematisch fokussierten LISA) macht das Programm für alle Gründer zugänglich, sofern sie entsprechenden Technologie- und Innovationsgehalt und die geforderten Wachstums- und Skalierungsmöglichkeiten nachweisen können.

- Durch die ausgeprägte Technologieorientierung bietet das Programm Potential auch als Katalysator für Förderung von Key Enabling Technologies unter Horizon 2020 zu dienen. Es ist anzunehmen, dass in Österreich in den nächsten Jahren Unternehmen gegründet werden, die in diesen Technologiefelder innovative Lösungen anbieten. JITU bietet ihnen die Möglichkeit diese Lösungen zu verwirklich und bietet damit auch die Chance, dass sie gleichsam "fit“ für eine Teilnahme an den entsprechenden EU-Förderungen gemacht werden.

- Insbesondere bei Projekten im Bereich IT / Mobile sind die Geschwindigkeit der Umsetzung der Marktidee und die möglichst rasche Skalierung (kurzfristige Time-to-Market) vielfach von entscheidender Bedeutung. Es ist daher anzustreben, dass die Zeit zwischen Förderansuchen, Förderentscheidung / Förderzusage und Auszahlung erster Fördermittel auf diesem Umstand entsprechend Rücksicht nimmt. 


\section{Empirische Analyse auf Basis des Programm-Monitorings}

In diesem Abschnitt werden die Entwicklungen der vier Förderprogramme PreSeed, Seedfinancing, Management auf Zeit und i2-Business Angels dargestellt. Innerhalb der Programme PreSeed und Seedfinancing wird dabei zwischen den Programmtypen IKT \& Physical Science und Life Science Austria (LISA) aus den in Abschnitt 1 erläuterten Gründen unterschieden. Die Auswertungen der Programmdaten der aws werden für die Programme Seedfinancing, MaZ und i2-Business Angel um Interviews mit geförderten bzw. vermittelten Unternehmen und Business Angels ergänzt. Innerhalb des PreSeed Programms wurde von Seiten der aws eine Sondererhebung bezüglich des Gründungsstatus der geförderten Projekte durchgeführt, welche ebenfalls in die Analysen mit einfließen. Schließlich werden auch die aws Portfolio-Monitoring-Daten über geförderte PreSeed und Seedfinancing Unternehmen analysiert. ${ }^{6}$ Diese Quellen stellen gemeinsam die Basis für die quantitative empirische Evaluierung der JITU-Programme und i2 dar. Die qualitative Beurteilung der PreSeed und Seedfinancing Projekte auf Basis einer OnlineBefragung bzw. einer internen aws-Evaluierung finden sich in Abschnitt 4.

Der gesamte JITU Programmverlauf (Summe aus PreSeed und Seedfinancing, exklusive MaZ) ist in Abbildung 13 dargestellt. Da im Jahr 2007 die Verhandlungen für den Ausführungsvertrag nicht rechtzeitig abgeschlossen werden konnten und deshalb keine LISA bzw. IKT\&PHS Seedfinancing Projekte zugesagt wurden, ergibt sich der Sprung zwischen 2007 und 2008 in der Zeitreihe. Zwischen 2008 und 2013 stieg die Anzahl der zugesagten Projekte (Life Science und IKT \& Physical Science) von 32 auf 49 an. Die damit korrespondierenden zugesagten Mittel stiegen in diesem Zeitraum von 11,5 auf 19,1 Mio. Euro an.

Abbildung 13: Programmverlauf JITU, 2007-2013
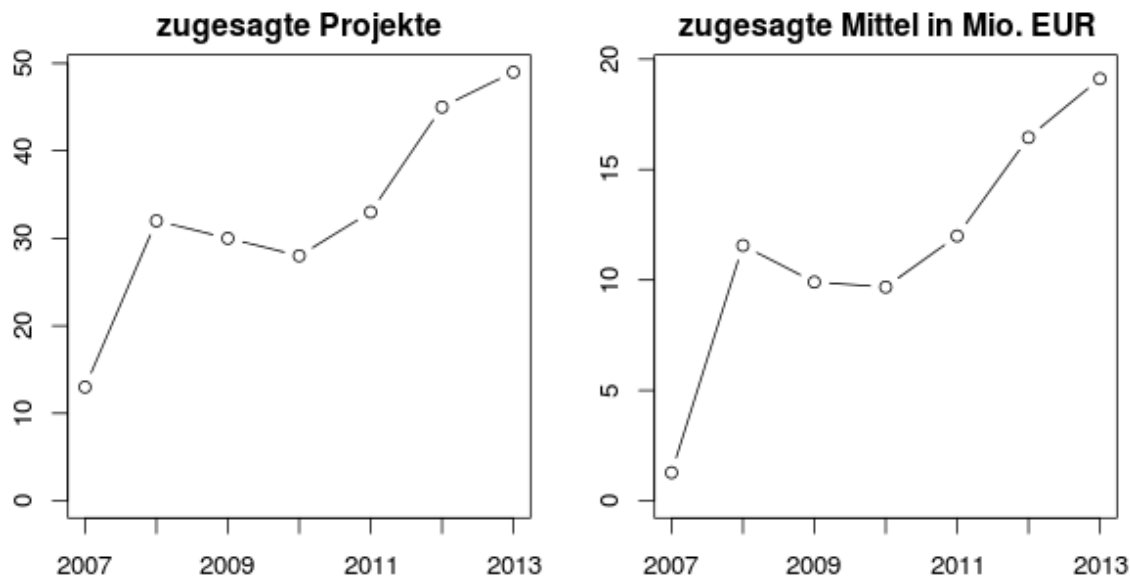

Quelle: aws, eigene Darstellung.

Im Folgenden werden die Programmverläufe von PreSeed und Seedfinancing für die Schwerpunkte Life Science (LISA) und IKT \& Physical Science aufgrund der Heterogenität der Projekte jeweils getrennt dargestellt. Dabei sollte beachtet werden, dass hierdurch sehr geringe Fallzahlen für zugesagte Projekte/Mittel auftreten können, welche im Zeitverlauf starken Schwankungen unterliegen können. Diese Schwankungen müssen nicht genuinen Entwicklungstrends im Gründungsgeschehen entsprechen sondern können auch auf zufälligen Ereignissen bzw. auf Besonderheiten der operativen Förderpraxis (periodische Abgrenzung, Zeitpunkt der Gremienentscheidungen, usw.) beruhen.

\footnotetext{
${ }^{6}$ Siehe auch Gassler, Ploder und Kleb (2013).
} 


\subsection{PreSeed}

Das Programm PreSeed besteht für Life Science Bereich seit 2003. Für den Schwerpunkt IKT \& Physical Science wurde PreSeed mit 2005 vom damaligen BMWFJ initiiert, wobei die ersten Förderungen mit 2006 begannen. Der Fokus dieser Evaluierung liegt auf der Programmentwicklung innerhalb der JITU-Richtlinie, welche mit 2007 in Kraft trat. Daher werden im Folgenden die Programmverläufe zwischen 2007 und 2013 dargestellt. Im Jahr 2010 stellte das Wirtschaftsministerium weitere 3 Mio. Euro für den einmaligen „PreSeed Sondercall 2010“ zur Verfügung. Dieser Call legte den Fokus auf die Förderung innovativer wissensbasierter Dienstleistungen zur Schaffung von Beschäftigung und Wachstum.

PreSeed - IKT \& Physical Science

Abbildung 14 zeigt die Entwicklung der eingereichten Anfragen, zugesagten Projekte und Mittel sowie die durchschnittliche Förderhöhe pro Förderfall des PreSeed Programms innerhalb des Schwerpunktes IKT \& Physical Science. Die genauen Daten können der Tabelle 12, Tabelle 14 und Tabelle 17 im Anhang entnommen werden. Mit Ausnahme des Jahres 2011 zeigt sich eine stetig steigende Anzahl an Anfragen von Unternehmen. So kam es zwischen 2007 und 2013 fast zu einer Verdoppelung an Anfragen (46 vs. 90). Pro Jahr wurde davon zwischen 11 und 19 Projekten eine Förderzusage erteilt. Im gesamten JITU-Zeitraum 2007-2013 wurden insgesamt 100 Projekte gefördert. Diese wiesen zugesagte Mittel in Summe von 13,6 Mio. Euro auf. Im Zeitverlauf schwankt die Höhe der zugesagten Gesamtmittel zwischen 1,3 und 2,6 Mio. Euro - in Abhängigkeit der Anzahl an zugesagten Projekten. Die durchschnittliche Förderhöhe pro Unternehmen betrug bis 2008 etwa 100.000 Euro - welches der maximalen Förderhöhe bis zum 4. Quartal 2009 entsprach. Danach wurde die maximale Fördersumme auf 200.000 Euro erhöht, was sich auch klar im Verlauf der durchschnittlichen Förderhöhe zeigt. Im Jahr 2010 betrug diese etwa 190.000 und sank dann auf zwischen 140.000 und 150.000 Euro in den Jahren 2011 bis 2013. 


\section{Abbildung 14: PreSeed IKT\&PHS Programmentwicklung}
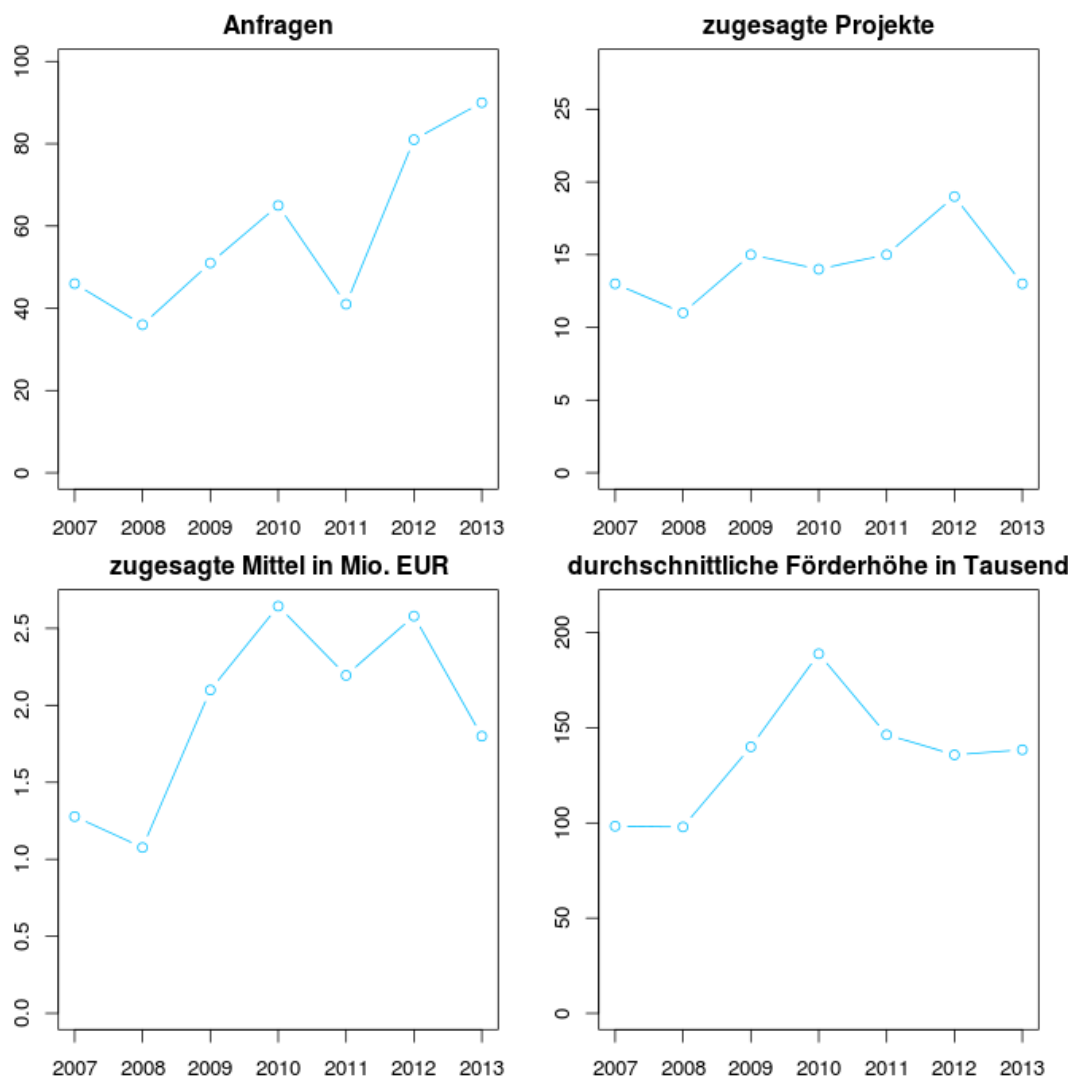

Quelle: aws, eigene Darstellung.

Im Schwerpunkt IKT \& Physical Science des PreSeed Programms lagen für die Jahre 2012 und 2013 die regionale Verteilung der Anfragen vor. Abbildung 15 zeigt die prozentuell Verteilung der Anfragen dieser beiden Jahre auf die Bundesländer. In beiden Jahren liegt Wien mit etwa $40 \%$ an erster Stelle, gefolgt von der Steiermark und Oberösterreich. Diese Verteilung zeigt einerseits einen geographischen Distanz Effekt und zum anderen weist sie auf die regionale Verteilung der Hochtechnologie-Industrie und der KMU generell in Österreich hin.

Abbildung 15: Regionale Verteilung der PreSeed IKT\&PHS Anfragen, 2012 und 2013

2012

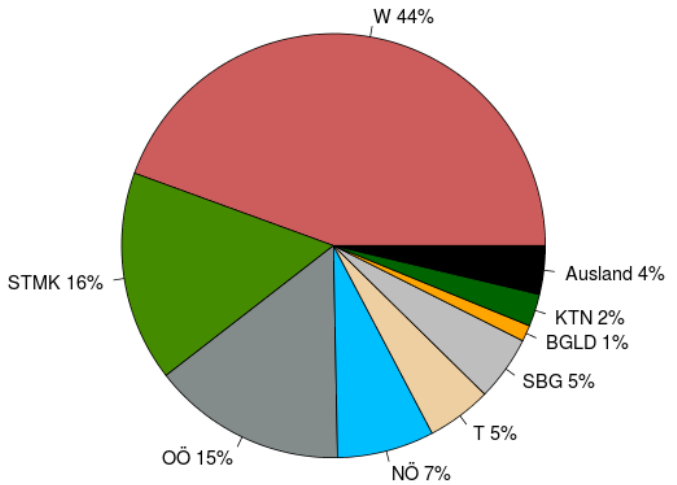

Quelle: aws, eigene Darstellung.
2013

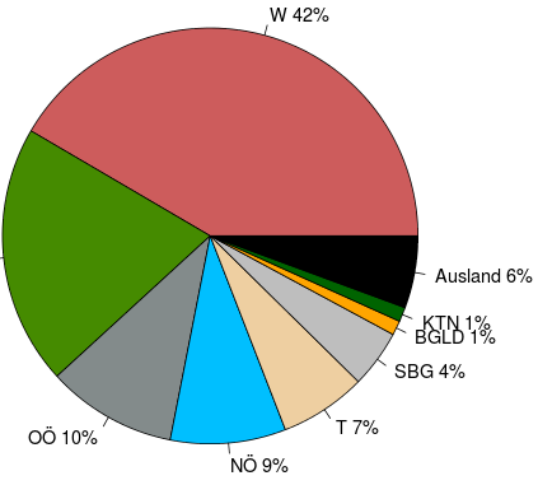


Die geringste Anzahl an Anträgen weisen das Burgenland, Kärnten und Vorarlberg auf, wobei aus letzterem Bundesland keine Anfrage eintraf. Interviews mit Programmverantwortlichen (JITU), Gründerzentren und Venture Capital Fonds Managern weisen darauf hin, dass dies an der starken Orientierung Vorarlbergs an der Schweiz und dem Süddeutschen Raum liegen könnte.

PreSeed - Life Science Austria (LISA)

Abbildung 16 zeigt die Anzahl der Projektzusagen und insgesamt zugesagten Mittel des PreSeed Programms im Life Science Bereich. Die entsprechenden Werte können in Tabelle 12 und Tabelle 17 im Anhang gefunden werden. Im Jahr 2007 wurden die Verhandlungen über die Richtlinien und den Ausführungsvertrag nicht rechtzeitig abgeschlossen, weswegen es in diesem Jahr keine formal zugesagten Projekte und Fördermittel gab. Im JITU-Zeitraum (2007-2013) wurde für insgesamt 38 LISA PreSeed Projekte eine Zusage erteilt, wobei die Anzahl zwischen 3 und 10 pro Jahr schwankt. Diese Schwankungen können letztlich auf die geringen Fallzahlen zurückgeführt werden. Der Verlauf der Projektzusagen spiegelt sich auch in der Höhe der für alle Projekte insgesamt zugesagten Mittel wieder. Diese betrugen 2008 etwa 500.000 und 2013 etwa 2 Mio. Euro. Abbildung 17 zeigt die sich aus diesen beiden Indikatoren ergebende durchschnittliche Förderhöhe pro Zusage. Wie schon zuvor im Schwerpunkt IKT \& Physical Science, schlägt sich hier die Erhöhung der maximalen Fördersumme auf 200.000 Euro pro Projekt durch.

\section{Abbildung 16: PreSeed LISA Programmentwicklung}
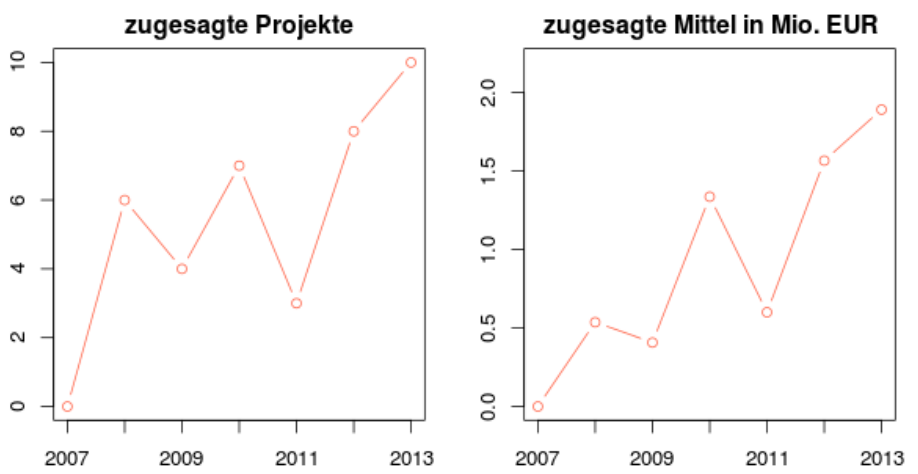

Quelle: aws, eigene Darstellung.

\section{Abbildung 17: PreSeed LISA - durchschnittliche Förderhöhe pro Zusage}

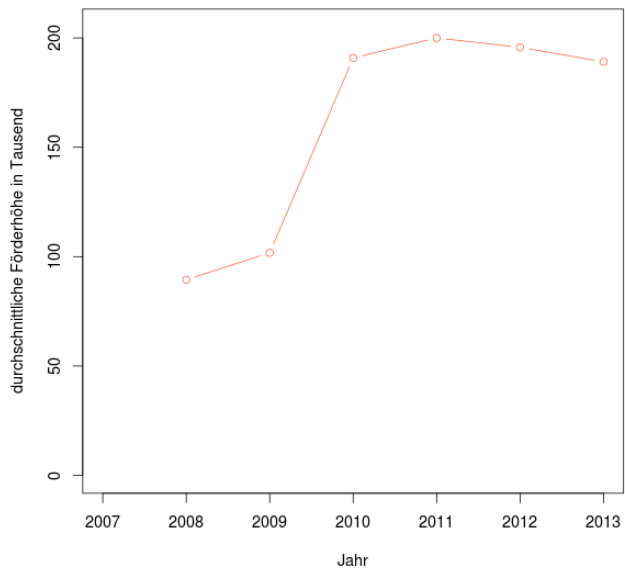

Quelle: aws, eigene Darstellung. 
Im Gegensatz zum Programmschwerpunkt IKT \& Physical Science liegt im LISA Bereiche keine getrennte Erfassung der Anfragen zwischen PreSeed und Seedfinancing vor. Tabelle $14 \mathrm{im}$ Anhang zeigt, die Summe der PreSeed und Seedfinancing Anfragen des Programmtyps LISA. Pro Jahr treffen zwischen 40 und 64 Anfragen ein, was in Summe über den JITU-Zeitraum etwa 350 Anfragen ergibt.

\subsection{Analyse PreSeed Gründungsdaten}

Die Datengrundlage der folgenden Analysen bildet eine interne aws Recherche über den Gründungsstatus von PreSeed Projekten zwischen 2006 und 2013. Mit Stichtag 19.08.2014 wurde erhoben ob eine Unternehmensgründung vorliegt. Dies beinhaltet zum einen Projekte welche derzeit noch aktiv in aws Betreuung befinden und auch Projekte welche seitens der aws bereits abgeschlossen sind. Abbildung 18 zeigt die Anzahl der Projekte nach dem Entscheidungsjahr (damit wird das Jahr bezeichnet in welchem die aws entschieden hat das Projekt zu fördern) sowie nach dem Programmtyp. Die Daten dahinter können aus Tabelle 15 im Anhang entnommen werden. PreSeed IKT wird mit "PSI" abgekürzt und ist in blau dargestellt, Projekte des Programmtyps LISA werden unter „PSL“ in Rot wiedergegeben und der einmalige PreSeed Call aus dem Jahr 2010 ist in orange mit "PSC" enthalten. Da PreSeed Projekte des Typs LISA erst mit 2008 hinsichtlich der Auszahlungen von der HighTech Förderung des aws übernommen wurden, starten die LISA PreSeed Projekte auch mit diesem Datum. Wie bereits weiter oben für LISA und IKT \& Physical Science gesehen werden konnte, stiegen die PreSeed Projekte insgesamt über den Zeitverlauf an.

\section{Abbildung 18: Projekte nach Entscheidungsjahr und Programmtyp}

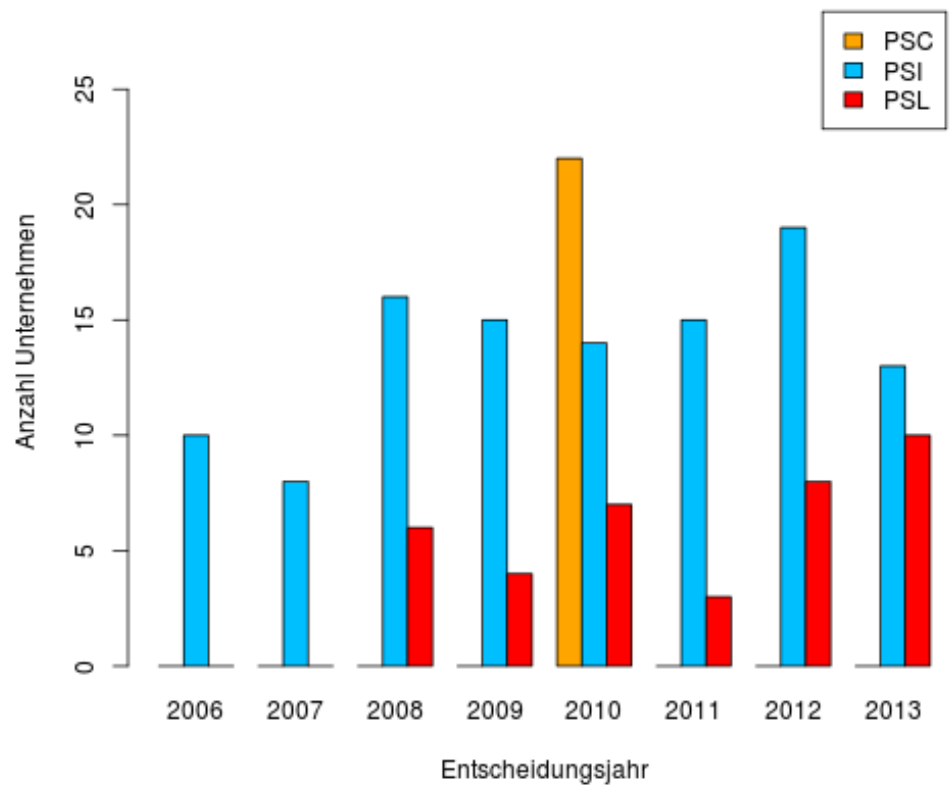

Quelle: aws, eigene Darstellung.

Abbildung 19 zeigt die durchschnittliche Förderhöhe nach Entscheidungsjahr und Programmtyp. Die Verläufe decken sich mit den oben beschriebenen Entwicklungen, wobei erkenntlich wird, dass die durchschnittliche Förderhöhe von Projekten des PreSeed Calls 2010 welche dem Programmtyp IKT \& Physical Science zuzurechnen sind, unterhalb vergleichbarer Projekte (in blau) außerhalb des Calls liegen. 


\section{Abbildung 19: Durchschnittliche Förderhöhe nach Entscheidungsjahr und Programmtyp}

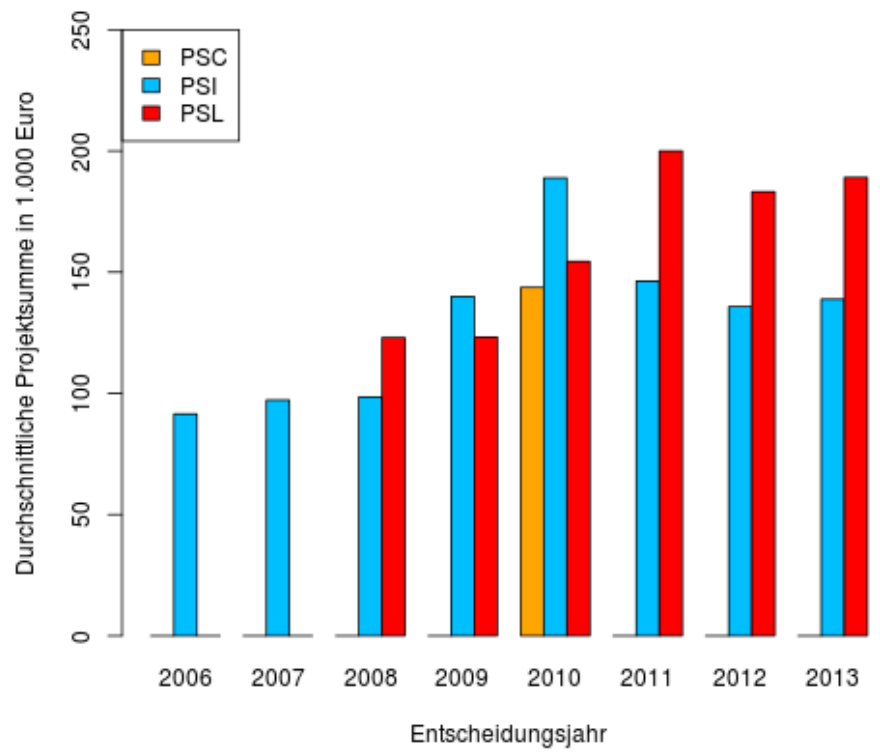

Quelle: aws, eigene Darstellung.

Im Weiteren wird von (erfolgreichen) Gründungen gesprochen, wenn mit Erhebungsstichtag eine Kapitalgesellschaft (GmbH oder AG) gegründet wurde, da dies letztlich das Ziel des Programms PreSeed ist. ${ }^{7}$ Fehlgeschlagene Gründungen, also abgebrochene Projekte in welchen die Zahlung der aws eingestellt wurden oder vertragsgemäß beendete Projekte bei welchen keine Gründung mehr erfolgen wird, konnten in der Recherche nicht hinreichend festgestellt werden. So wurden zwar 4 definitive Abbrüche identifiziert, jedoch konnte nicht generell festgestellt werden ob es sich bei jenen Projekten für die zum Stichtag noch keine Firmenbucheintragung vorlag, um einen sicheren Abbruch handelt. Daher werden all jene Projekte für welche zum Stichtag keine Kapitalgesellschaftsgründung vorlag im Folgenden mit „noch keine Gründung“ bezeichnet.

Abbildung 20 stellt die erfolgreichen Gründungen den noch nicht erfolgten (inkl. der gescheiterten) Gründungen gegenüber. Aufgrund des außerordentlichen und einmaligen PreSeed Calls 2010 weisen die meisten Gründungen zum Erhebungsstichtag das Entscheidungsjahr 2010 auf. Einzig im Jahr 2013 überwiegt die Anzahl noch nicht gegründeter Unternehmen. Das liegt zum Teil daran, dass sich viele dieser Projekte noch im Vertragsmanagement befinden und diese Unternehmen per Definition zum Erhebungsstichtag die jüngste Kohorte abbilden.

\footnotetext{
7 Hierbei sei auf eine Diskrepanz zwischen den hier präsentierten Gründungsdaten des PreSeed Calls 2010 und den in der Broschüre „PreSeed Call - Geförderte Start-ups, BMWFJ (2013)“ enthaltenen Zahlen hingewiesen. In der vorliegenden Studie werden lediglich Gründungen von Kapitalgesellschaften gewertet, während die Broschüre alle Unternehmensformen $(\mathrm{OG}, \mathrm{KG}, . .$.$) als Gründungen$ auffasste.
} 


\section{Abbildung 20: Gründungsstatus nach Entscheidungsjahr}

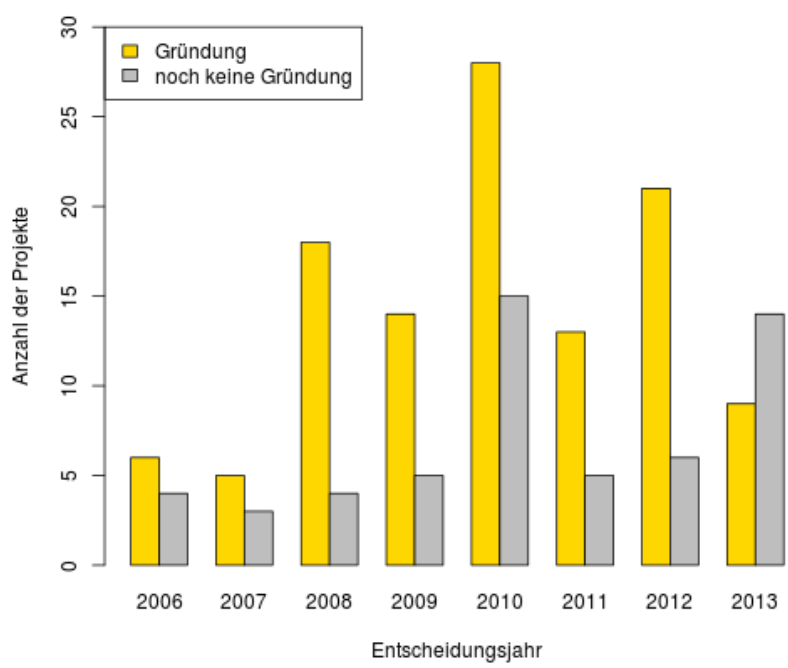

Quelle: aws, eigene Darstellung.

Abbildung 21 zeigt den Anteil der erfolgten Gründungen jedes Entscheidungsjahres bezogen auf alle eingereichten Projekte dieses Jahres. Diese „Gründungsquote“ oder „Erfolgsquote“ schwankt über die Jahre zwischen 60 und 80 Prozent, was ziemlich genau dem Zielindikator des PreSeed Programms diesbezüglich entspricht. Exkludiert man die zwei jüngsten Kohorten (also die Entscheidungsjahre 2012 und 2013) so ergibt sich für den Zeitraum 2006-2011 eine Quote von 70\%. Betrachtet man den Gesamtzeitraum 20062013 und bereinigt um die 4 aus der Erhebung identifizierten Abbrüche, so ergibt sich eine Gründungsquote von 69\%. Kontrastiert man diese Quote mit den 50\% erfolgreich gegründeten PreSeed Call 2010 Projekten, wären die Projekte des PreSeed Calls bis dato seltener erfolgreich hinsichtlich einer erfolgreichen Gründung als Projekte der anderen Themenschwerpunkte.

\section{Abbildung 21: Gründungsquote nach Entscheidungsjahr (und Programmtyp)}
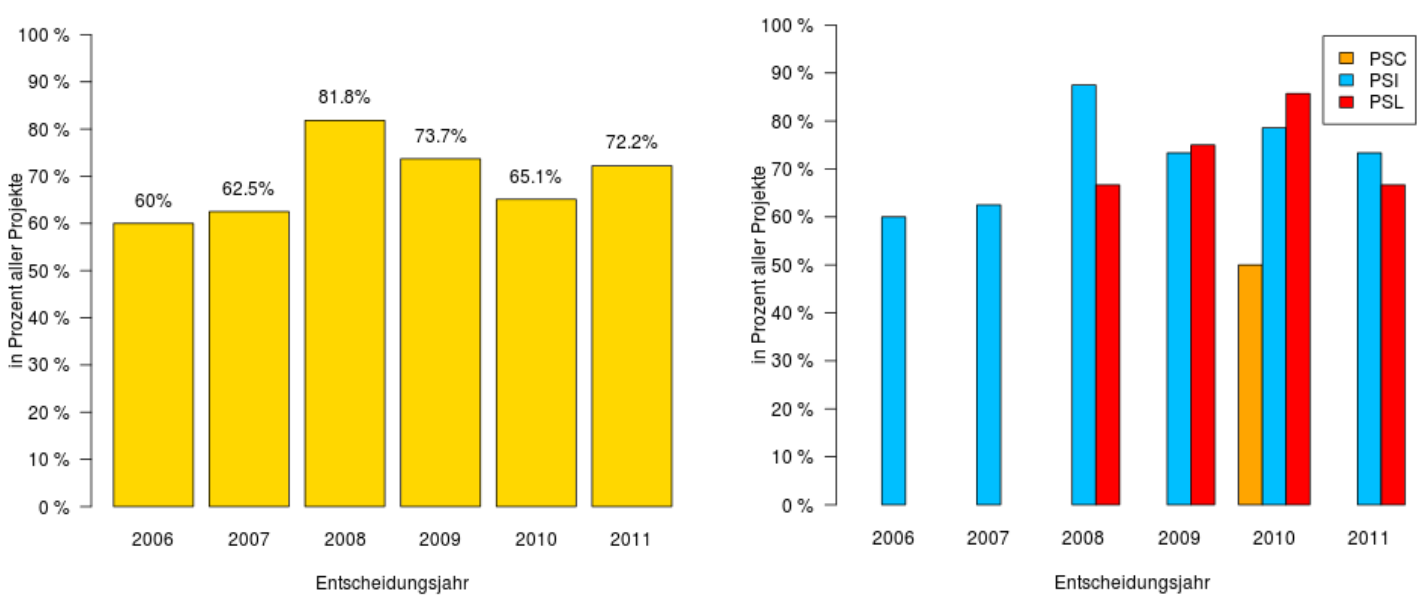

Quelle: aws, eigene Darstellung.

Die erhobenen Daten beinhalten den Tag der Entscheidung der aws das Projekt zu fördern sowie den Tag der Eintragung ins Firmenbuch. Daraus lässt sich die genaue Gründungsdauer ermitteln. Abbildung 22 zeigt die Verteilung der Gründungsdauer aller insgesamt 114 erfassten Gründungen. Die Balken geben die empirische Verteilung und die Dichtekurve die mittels Kerndichteschätzung ermittelte geglättete Version der Gründungsdauer wieder. Man sieht, dass die Verteilung im Bereich um 360 Tage die höchste Dichte 
aufweist. Zudem findet sich ein Unternehmen unter den PreSeed Projekten, dass etwa 2.000 Tage bis zur Gründung benötigt hat.

\section{Abbildung 22: Verteilung der Gründungsdauer, insgesamt}

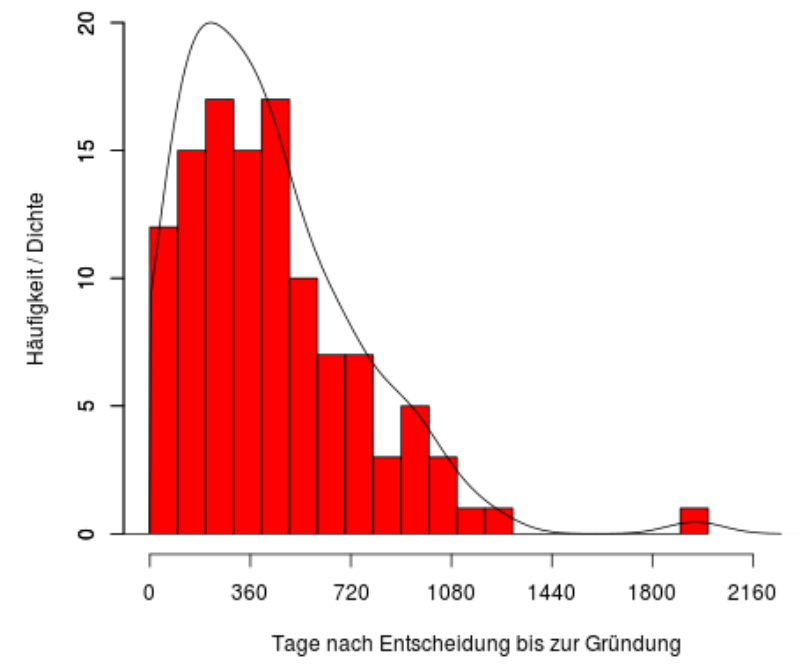

Quelle: aws, eigene Darstellung.

Abbildung 23 und Tabelle 2 zeigen die zentralen statistischen Maße der Verteilung der Gründungsdauer nach Programmtyp. Die untere und obere Antenne in Abbildung 23 geben den Minimum- bzw. Maximumwert (ohne Ausreißer) an. Die Box umfasst die mittleren 50\% der Beobachtungen und der dicke schwarze Strich markiert den Median. Wie aus Tabelle $\mathbf{2}$ ersichtlich, beträgt die durchschnittliche Gründungsdauer über alle Gruppen im Median (das wäre das mittlere Unternehmen) ziemlich genau 1 Jahr (376 Tage), was der typischen Laufzeit von PreSeed laut Programmdokumentation entspricht. Die Gründungsdauer der erfassten LISA Projekte liegt mit etwa 200 Tagen deutlich unter jener der Projekte anderer Schwerpunkte. Die mittlere Hälfte aller Gründungen (1. bis 3. Quantil) weist eine Gründungsdauer von zwischen 200 und 600 Tagen auf.

Die Gründungsdauern schwanken innerhalb der Programmtypen zum Teil erheblich. Vereinzelt finden sich sehr lange (über mehrere Jahre) dauernde Gründungsprozesse. Eine mögliche Ursache könnte die hohe technologische Komplexität der Projekte bzw. in der Änderung der ursprünglichen Geschäftsidee bzw. des modells („Pivoting“). Dies zeigte sich auch in einigen der qualitativen Interviews.

\section{Abbildung 23: Verteilung der Gründungsdauer nach Programmtyp}
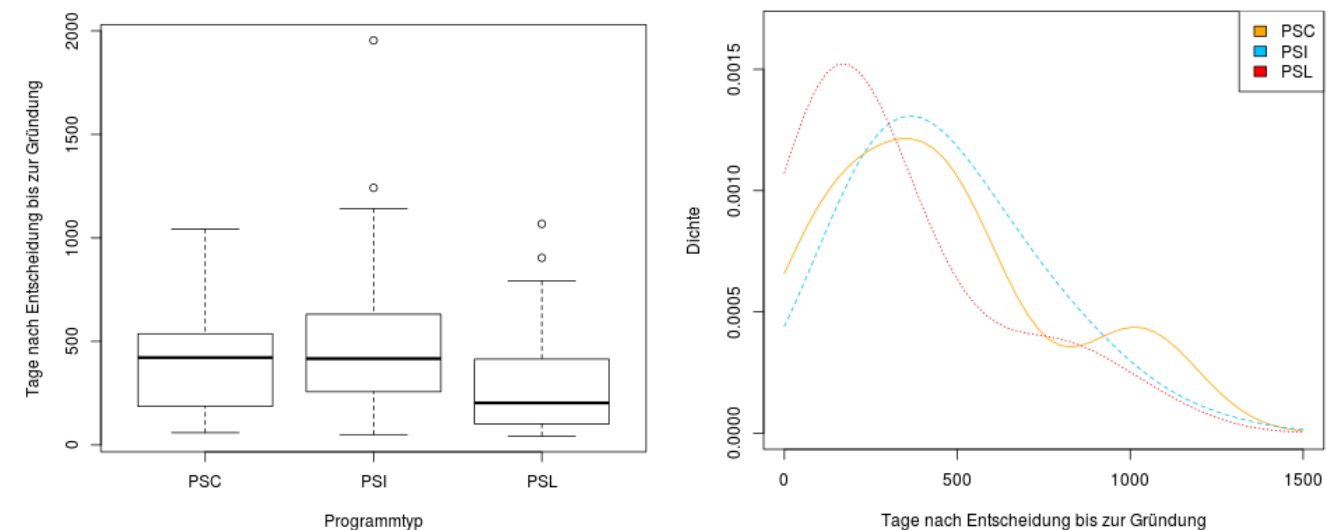

Quelle: aws, eigene Darstellung. 
Tabelle 2: Verteilung der Gründungsdauer nach Programmtyp

\begin{tabular}{lrrrrrrr} 
Typ & Max. & erstes Quantil & Durchschnitt & Median & drittes Quantil & Min. & Anzahl \\
\hline PSC & 1043 & 537 & 444 & 422 & 186 & 59 & 263 \\
PSI & 1954 & 630 & 483 & 417 & 50 & 11 \\
PSL & 1068 & 415 & 318 & 203 & 27 & 43 \\
\hline Alle & 1954 & 597 & 440 & 376 & 204 & 43 \\
\hline
\end{tabular}

Quelle: aws, eigene Darstellung.

\section{Abbildung 24: Verteilung der Gründungsdauer nach Entscheidungsjahr}

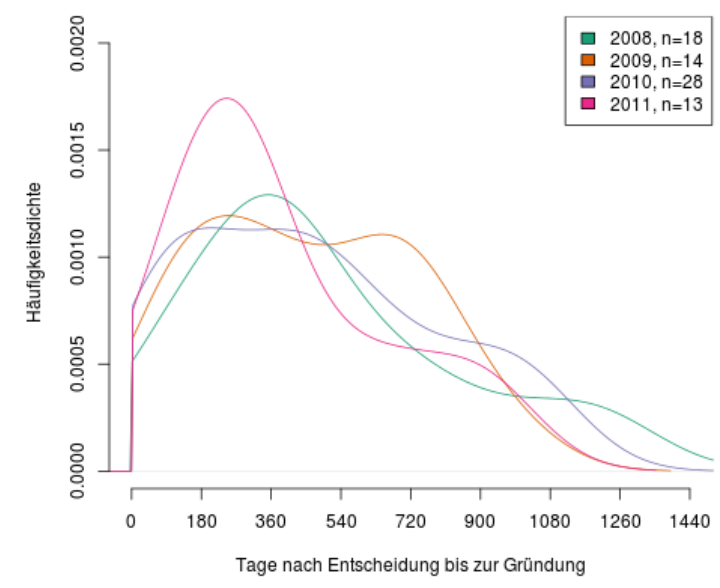

Quelle: aws, eigene Darstellung.

Abbildung 24 zeigt die Verteilung der Gründungsdauer nach dem Entscheidungsjahr der Förderung (wobei die letzten beiden Kohorten exkludiert wurden). Da hier nur eine geringe Anzahl an Beobachtungen zur Verfügung stand, sollte den Ergebnissen nicht allzu viel Gewicht beigemessen werden. Projekte welche im Jahr 2011 angenommen wurden weisen in der Tendenz eine geringere Gründungsdauer, mit einem Masseschwerpunkt auf etwas mehr als einem halben Jahr, auf. Die Gründungsdauer der Projekte bei welchen die Entscheidung der Förderung im Krisenjahr 2009 getroffen wurde weist hingegen eine bimodale Verteilung mit einem zusätzlichen Peak bei etwa zwei Jahren.

Diese schwache Tendenz zu sinkenden Gründungsdauern im Beobachtungszeitraum, lässt sich auch bei Betrachtung der einzelnen Projekte im Zeitablaufdiagramm in Abbildung $\mathbf{2 5}$ erkennen. Auf der y-Achse sind hierbei die Unternehmen nach Entscheidungsdatum sortiert (jeder Balken entspricht einem Projekt) und auf der $x$-Achse sind die Gründungsdauern (Verlauf: Entscheidungsdatum bis Gründungsdatum) aufgetragen. Wie bereits zuvor festgehalten wurde, lässt sich hier auch erkennen, dass viele Projekte mit Entscheidungsdatum beginnend ab 2012 bereits erfolgreich gegründet sind. 
Abbildung 25: Gründungsdauern der einzelnen Projekte ( $n=114)$

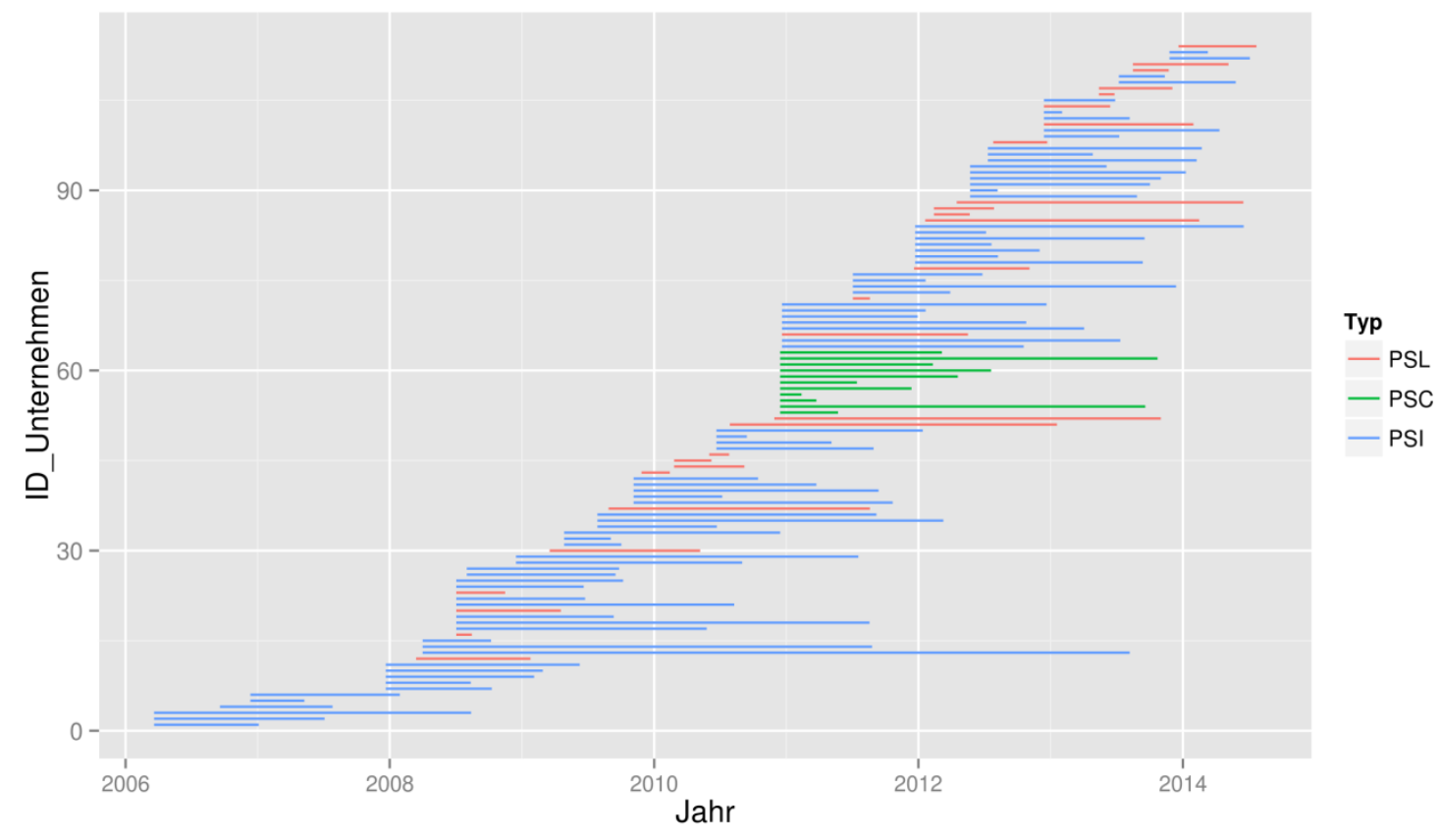

Quelle: aws, eigene Darstellung.

\subsection{Seedfinancing}

Das Seedfinancing Programm ist das älteste in dieser Studie evaluierte Programm der JITU-Förderschiene. Im IKT \& Physical Science Bereich besteht das Programm bereits seit 1989 (im Rahmen des ITF-Fonds), der Schwerpunkt Life Science Austria kam 1998 hinzu. Aus dem Leistungsbericht der aws des Jahres 2011 und zusätzlich bereitgestellten Aktualisierungen der aws lässt sich der Seedfinancing Programmverlauf beginnend mit 1995 darstellen. Abbildung 26 zeigt die Anzahl der geförderten Unternehmen, deren Umsätze und Mitarbeiter im Zeitverlauf. Die entsprechenden Daten können der Tabelle 15 im Anhang entnommen werden. Die Anzahl der insgesamt geförderten Unternehmen stieg von $71 \mathrm{im}$ Jahr 1995 auf $260 \mathrm{im}$ Jahr 2013 an. Dadurch wurden im Jahr 2013 knapp 2.500 Mitarbeiter beschäftigt. Die Umsätze aller Seedfinancing geförderten Unternehmen wuchsen von 14 Mio. Euro im Jahr 1995 auf 241 Mio. Euro im Jahr 2013 an. 
Abbildung 26: Entwicklung Seedfinancing, 1995-2013

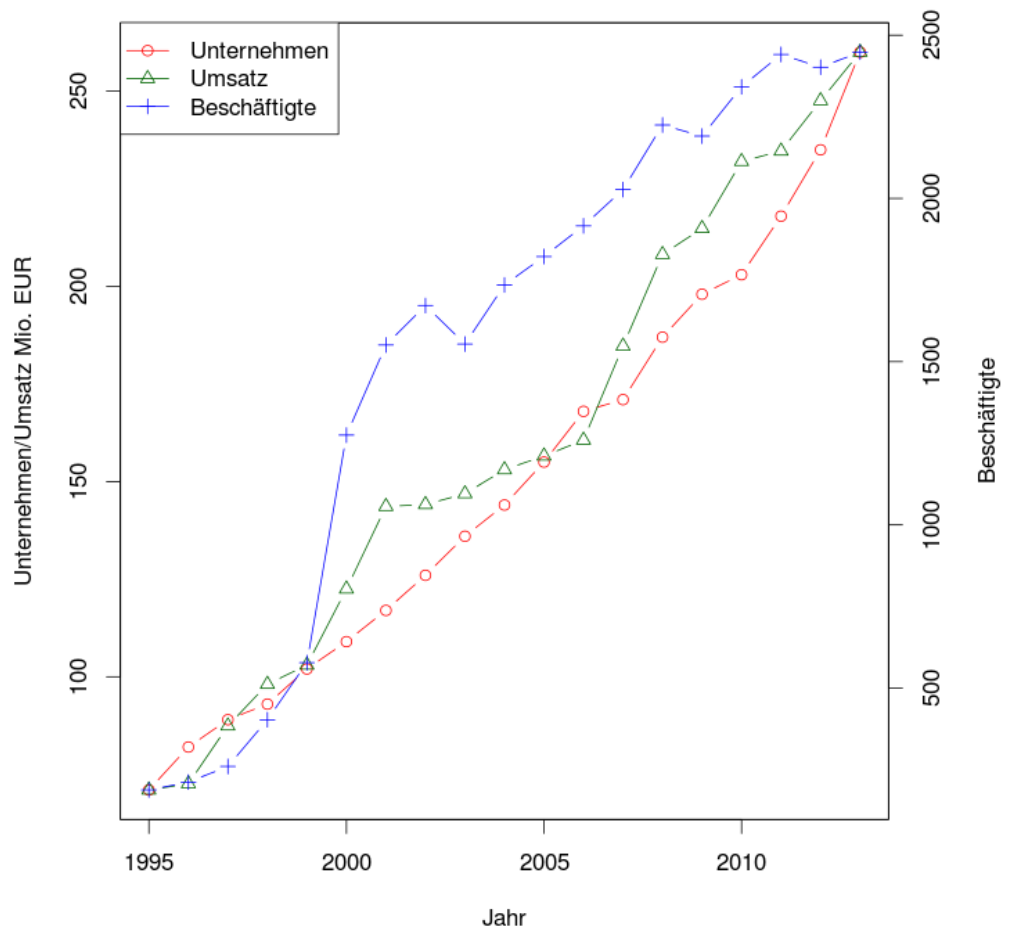

Quelle: aws/erp-fonds (2012), eigene Darstellung.

In Tabelle 3 sind neben den insgesamt geförderten Unternehmen auch die in dem jeweiligen Jahr im awsPortfolio befindlichen Unternehmen enthalten. Diese wuchsen zwischen 2002 und 2013 von 70 auf 111 an. Der Wert der sich im Portfolio befindenden Unternehmen wuchs von etwa 21 Mio. auf etwa 48 Mio. Euro an. Die kumulierten Auszahlungen verdreifachten sich von 31 Mio. im Jahr 2002 auf 91 Mio. Euro in 2013. Mit 2013 wurden insgesamt 49 Highflyer $^{8}, 150$ Exits, 74 erfolgreiche Abschichtungen ${ }^{9}$ und 63 Insolvenzen gezählt. Zum Stand September 2014 betrug die kumulierte Höhe der Rückflüsse innerhalb des JITUProgramms 1,74 Mio. Euro. ${ }^{10}$

\footnotetext{
${ }^{8}$ Highflyer bedeutet die Erreichung eines Umsatzes von 4 Mio. Euro innerhalb von 5 Jahren ab Gründung oder Abschichtung mit maximaler Rendite.

${ }^{9}$ Eine erfolgreiche Abschichtung liegt vor wenn mindestens das von der aws eingesetzte Kapital (Mezzanindarlehen) bzw. der gewährte Zuschuss (JITU-Periode) zurückgezahlt wurde.

${ }^{10}$ Die Periode zwischen der Auszahlung der Förderung und dem Beginn der Rückzahlung unterscheiden sich zwischen den einzelnen Projekten mitunter stark. Daher wird von einer jährlichen Gegenüberstellung der Auszahlungen und Rückflüsse abgesehen.
} 
Tabelle 3: Portfoliodaten Seedfinancing, 2002-2013

\begin{tabular}{|c|c|c|c|c|c|c|c|c|}
\hline Jahr & Unternehmen & Portfoliounternehmen & Portfoliowert & $\begin{array}{c}\text { kumulierte } \\
\text { Auszahlungen }\end{array}$ & Highflyer & Exits & $\begin{array}{c}\text { erfolgreiche } \\
\text { Abschichtungen }\end{array}$ & Insolvenzen \\
\hline 2003 & 136 & 74 & 23.800 .000 & 35.435 .985 & 30 & 62 & 31 & 24 \\
\hline 2005 & 155 & 77 & 26.300 .000 & 43.404 .330 & 34 & 78 & 41 & 29 \\
\hline 2006 & 168 & 83 & 30.800 .000 & 49.764.330 & 34 & 85 & 45 & 32 \\
\hline 2007 & 171 & 80 & 31.100 .000 & 52.689 .330 & 36 & 91 & 48 & 35 \\
\hline 2010 & 203 & 93 & 33.700 .000 & 67.293 .424 & 41 & 110 & 55 & 47 \\
\hline 2011 & 218 & 95 & 36.900 .000 & 74.578 .424 & 46 & 123 & 63 & 51 \\
\hline 2012 & 235 & 101 & 41.411 .000 & 82.275 .924 & 48 & 134 & 68 & 57 \\
\hline 2013 & 260 & 111 & 47.505 .000 & 92.442 .424 & 49 & 149 & 74 & 63 \\
\hline
\end{tabular}

Quelle: aws, eigene Darstellung.

Bezieht man die 63 Insolvenzen auf die insgesamt 260 geförderten Seedfinancing Unternehmen so erhält man eine Insolvenzrate von $24 \% \mathrm{im}$ Jahr 2013. Tabelle 4 zeigt im Vergleich dazu die Insolvenzraten österreichischer Unternehmen 1 bis 7 Jahre nach Gründung. Demnach weisen aws-geförderte Unternehmen eine vergleichbare Insolvenzrate wie Unternehmen 3 Jahre nach der Gründung auf. ${ }^{11} \mathrm{Da}$ jedoch 2009 bereits etwa 200 der 260 Unternehmen bestanden, welche im Jahr 2013 bereits 4 Jahre alt waren, kann davon ausgegangen werden, dass Seedfinancing geförderte Unternehmen im Durchschnitt älter als 3 Jahre sind und demnach eine höhere Überlebenswahrscheinlichkeiten als ein typisches österreichisches Unternehmen aufweisen. Aus dieser Beobachtung lassen sich zwei Thesen ableiten. Einerseits könnte alleinig die Tatsache der finanziellen Förderung die Überlebenswahrscheinlichkeit gegenüber einem Durchschnittsunternehmen erhöhen. Andererseits handelt es sich bei den im Rahmen des Seedfinancing geförderten Unternehmen um riskante Hochtechnologieunternehmen, welche üblicherweise eine höhere Ausfallquote aufweisen. Eine niedrige Ausfallsquote könnte somit zum Teil auf den Selektionseffekt des Programms zurückzuführen sein („picking the winners“ bzw. „avoiding the losers“).

Tabelle 4: Insolvenzraten Unternehmen insgesamt vs. aws geförderte Unternehmen

\begin{tabular}{lr} 
nach ... & Insolvenzrate \\
\hline 1 Jahr & $10 \%$ \\
2 Jahren & $19 \%$ \\
3 Jahren & $25 \%$ \\
4 Jahren & $31 \%$ \\
5 Jahren & $36 \%$ \\
6 Jahren & $40 \%$ \\
7 Jahren & $44 \%$ \\
& \\
Insolvenzen / geförderte Unternehmen (aws, 2013) & $24 \%$ \\
\hline
\end{tabular}

Quelle: aws, Statistik Austria - Unternehmensdemographie (Überlebensraten 2004 gegründeter Unternehmen).

Tabelle 5 zeigt die Ergebnisse einer schon etwas älteren Befragung von Venture Capital Fonds Managern über ihre Einschätzung zum Verlustrisiko eines Investments basierend auf der Investmentphase. Hier zeigt sich der klassische Verlauf, dass das Ausfallsrisiko mit steigendem Unternehmensalter und sinkendem Entwicklungsrisiko fällt. Die Unternehmen des Seedfinancing Programm würden ihrer Phase und ihrem Risikogehalt nach in diesem Schema in die Kategorien Start-Up (Geschäftsmodell konzipieren, Marktanalysen, erste Beta-Test der Produkte) und tlw. Second Stage (Prototyp fertig, erste Umsätze,

\footnotetext{
${ }^{11}$ Informationen zum Unternehmensalter der Insolvenzen der geförderten Unternehmen standen zum Zeitpunkt der Erstellung der Studie nicht zur Verfügung steht.
} 
Marketing Push gebraucht) fallen. In diesen Kategorien schätzten die befragten 73 VC Manager das Ausfallsrisiko auf zwischen $34 \%$ und $53 \%$ ein.

Tabelle 5: Verlustrisiko Venture Capital nach Phasen

\begin{tabular}{lr} 
Point at which investment made & Risk of loss \\
\hline Seed stage & $66,2 \%$ \\
Start-Up stage & $53,0 \%$ \\
Second stage & $33,7 \%$ \\
Third stage & $20,1 \%$ \\
Bridge or pre-public stage & $20,9 \%$ \\
\hline
\end{tabular}

Quelle: übernommen aus Gerken (2014, 110) bzw. Ruhnka and Young (1987).

Ein weiterer Vergleich aus dem Bereich des Risikokapitals wäre eine Gegenüberstellung mit den DeInvestitions- bzw. Exitkanälen der Statistiken der European Venture Capital Association (EVCA). Die Kategorie "Write-Off“ also „Abschreibung“ wäre hier gleichzusetzen mit einem Ausfall der Investition. Hierunter fallen im Durchschnitt der Jahre 2007-2013 etwa 40\% der österreichischen Ventures, was verglichen mit Europa relativ hoch ist. Darin enthalten sind jedoch nicht nur die Frühphasen-Investments (Seed und Start-Up) sondern auch die VC Later-Stage („Expansionskapital“).

\section{Abbildung 27: Exit Kanäle (De-Investitionen) VC-finanzierte Unternehmen}

Österreich, Summe 2007-2013

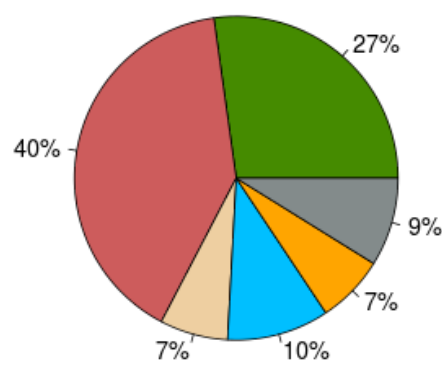

Europa, Summe 2007-2013

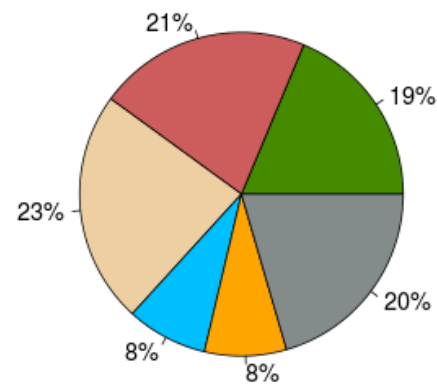

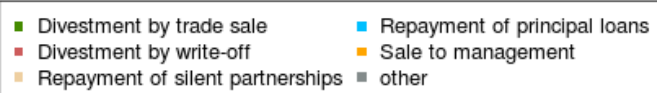

Quelle: EVCA Yearbook 2014, eigene Darstellung. Anmerkung: $n=147$ (AT) bzw. 1693 (Europa).

Zusätzlich zu den Auswertungen der vorhandenen internen Daten der aws wurden bei einschlägig geförderten Unternehmen qualitative Interviews durchgeführt. ${ }^{12}$ Grundsätzlich zeichnen diese qualitativen Ergebnisse ein sehr positives Bild. Ohne Seedfinancing wären die Umsetzung technologisch innovativer Ideen und der Unternehmensaufbau kaum möglich gewesen, da alternative Finanzierungsquellen in der Frühphase de facto nicht vorhanden sind. Die substanzielle Förderung gibt in den ersten Jahren (wo ja vielfach erst die Produktentwicklung erfolgt und keine bzw. kaum Umsätze vorhanden sind) die entsprechende Sicherheit, dass das Unternehmen nicht „mitten im Prozess der Produktentwicklung bzw. Markteinführung aufhören muss".

12 Im Sommer 2013 wurden in einer von dieser Evaluierung unabhängigen Studie ebenfalls mehrere qualitative Interviews mit Seedfinancing geförderten Unternehmen durchgeführt (Gassler et al., 2013). Die Erfahrungen aus diesen Interviews fließen hier ebenfalls mit ein. 
Auch die Projektbegleitung durch die jeweiligen Referenten der aws wird positiv betrachtet, da durch die laufenden Gespräche und Rückmeldungen nicht nur wertvolles Knowhow in das Unternehmen fließt, sondern auch der Status Quo und die Entwicklungsperspektive des Unternehmens von Seiten der Gründer analysiert werden muss, was wiederum wichtige Grundlagen für die weitere Entscheidungsfindung bzw. Vorgangsweise beim Unternehmensaufbau liefert.

Kritikpunkte werden teilweise an der Intensität des quartalsweisen Berichtwesens angebracht und in Frage gestellt, ob es tatsächlich notwendig sei, jedes Quartal Reports abzuliefern. Teilweise wird das als „bürokratischer" Aufwand gesehen. Ebenfalls wurde erwähnt, dass es bei der Auszahlung bereits bewilligter Förderungen manchmal zu Verzögerungen komme. Ein Medizintechnik-Unternehmen merkte an, dass die Zeitdauer zwischen den jeweiligen Milestones für die spezifische Branche zu knapp sei, da die entsprechenden (klinischen) Tests längere Laufzeiten aufweisen.

\section{IKT \& Physical Science}

In Abbildung 28 ist die Entwicklung des Seedfinancing Programmschwerpunktes IKT \& Physical Science im JITU-Zeitraum dargestellt. Die entsprechenden Werte können Tabelle 13, Tabelle 14 und Tabelle 17 im Anhang entnommen werden. Im Gesamtzeitraum 2007-2013 wurden 262 Anfragen von Unternehmen gestellt, 62 Projekten eine Zusage erteilt und in Summe 37,5 Mio. Euro zugesagt. Die Anfragen stiegen zwischen 2007 und 2013 relativ stetig von 33 auf 46 an. Auch im Seedfinancing gab es im Jahr 2007 aufgrund der nicht rechtzeitig abgeschlossenen Verhandlungen über die Richtlinien und den Auftragsvertrag keine formalen Zusagen. Die zugesagten Projekte entwickelten sich zwischen 2008 und 2010 rückläufig und stiegen dann bis 2013 auf zuletzt 21 an. Einen ähnlichen Verlauf zeigen die insgesamt zugesagten Mittel, welche 2013 bei 12 Mio. Euro lagen. Die durchschnittliche Förderhöhe für Unternehmen im Seedfinancing Schwerpunkt IKT\&PHS schwankt um 600.000 Euro. 
Abbildung 28: Seedfinancing IKT\&PHS Programmentwicklung
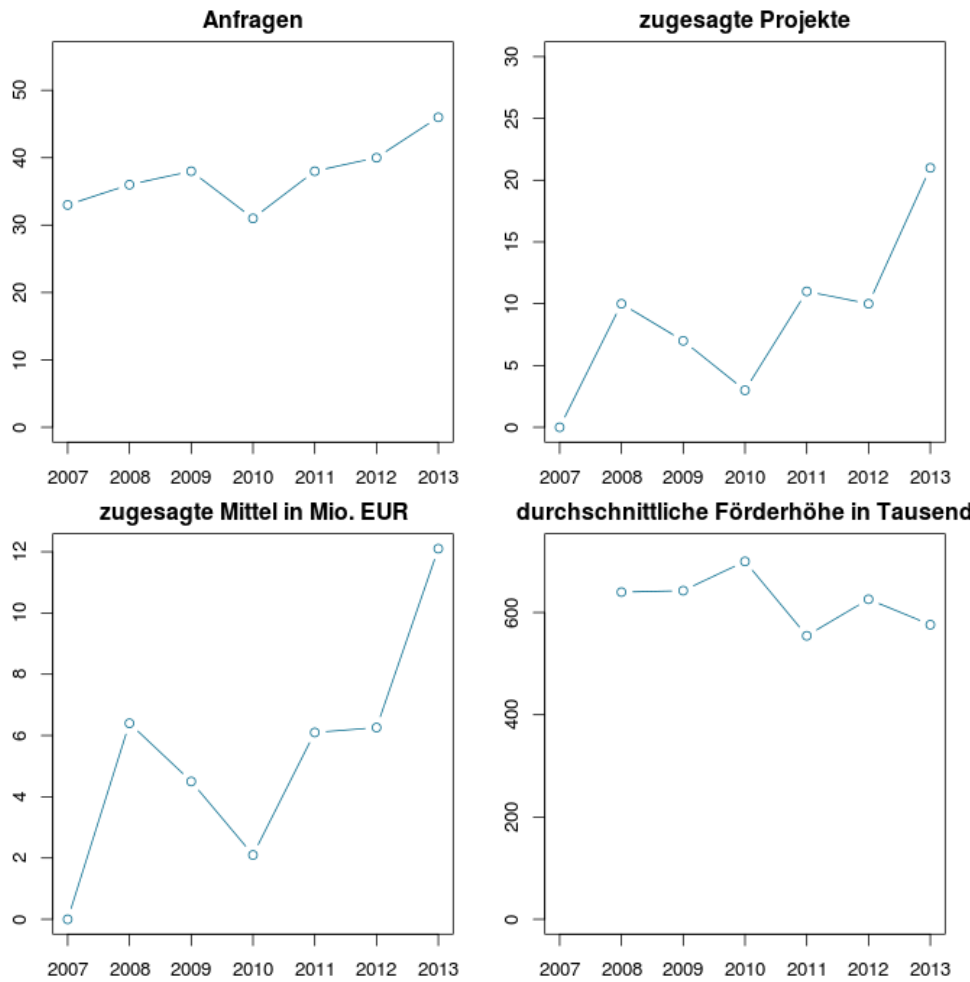

Quelle: aws, eigene Darstellung.

Abbildung 29 zeigt die regionale Verteilung der zugesagten Projekte zwischen 2007-2013. Hier zeigt sich ein ähnliches Muster wie zuvor bei den Anfragen im PreSeed Programm. Die meisten Zusagen entfallen auf Unternehmen in Wien, gefolgt von der Steiermark und Oberösterreich. Die Bundesländer Niederösterreich, Tirol, Salzburg und Burgenland sind nicht jedes Jahr mit Seedfinancing geförderten Unternehmen vertreten. Im Jahr 2013 waren gar nur Wien, die Steiermark und Oberösterreich am Seedfinancing Programm im Bereich IKT \& Physical Science vertreten. Aus Kärnten und Vorarlberg wurde seit Einführung des JITU Dachprogramms kein Unternehmen innerhalb des Seedfinancing in diesem Schwerpunkt gefördert. 
Abbildung 29: Regionale Verteilung der beschlossenen Seedfinancing Projekte in IKT\&PHS

Beschlossene Projekte, 2007-2013

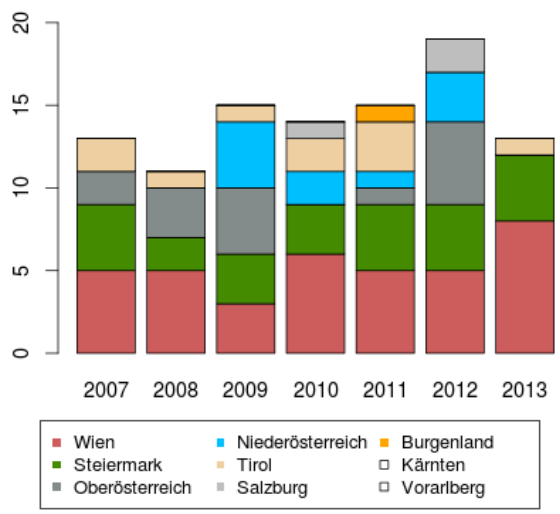

Quelle: aws, eigene Darstellung.
Regionale Verteilung, Summe 2007-2013

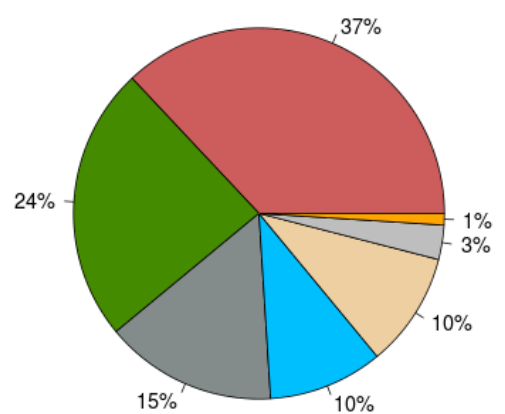

Life Science Austria

Abbildung 30 zeigt den zeitlichen Verlauf der zugesagten Projekte und Mittel im Seedfinancing Programmtyp Life Science. Die zugrunde liegenden Daten können Tabelle 13 und Tabelle 17 im Anhang entnommen werden. Innerhalb des JITU-Zeitraums wurden insgesamt 30 Projekte mit in Summe 22,5 Mio. Euro gefördert. Mit Ausnahme des Jahres 2008 erhalten etwa 4-5 LISA Projekte pro Jahr eine Seedfinancing Zusage. Die für die 5 Projekte in 2013 zugesagten Mittel betrugen 3,3 Mio. Euro. Die durchschnittliche Förderhöhe (siehe Abbildung 31) schwankte zwischen 665.000 und 900.000. Dieser Wert liegt deutlich über der durchschnittlichen Förderhöhe der IKT \& Physical Science Projekte, da im Life Science Bereich meist mehrere Auszahlungsrunden über einen längeren Zeitraum geleistet werden und höhere Fixkosten für Laborausstattungen und auch die Kosten für klinische Testphasen bereitgestellt werden müssen.

\section{Abbildung 30: Seedfinancing LISA Programmentwicklung}
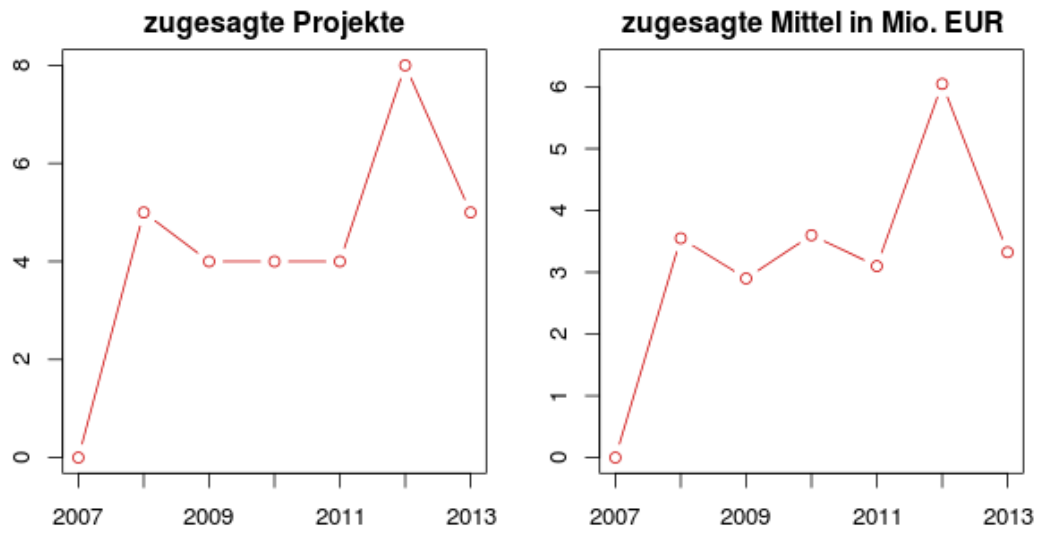

Quelle: aws, eigene Darstellung. 


\section{Abbildung 31: Seedfinancing LISA - durchschnittliche Förderhöhe pro Zusage}

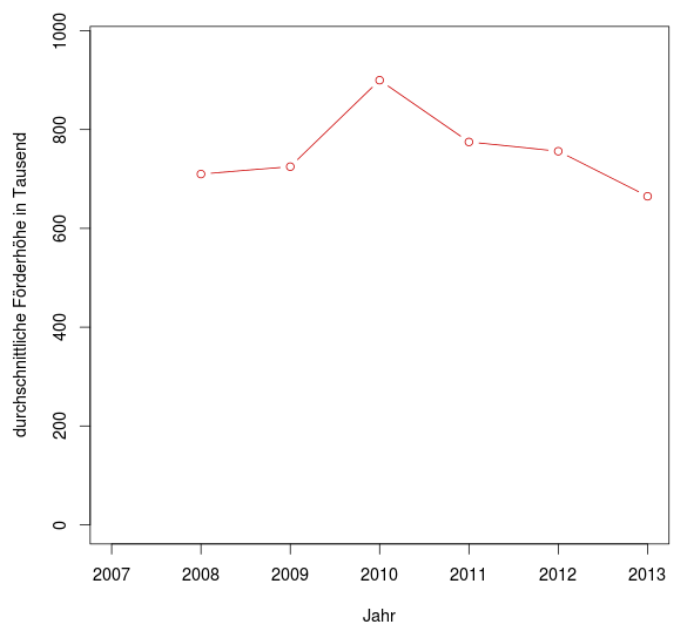

Quelle: aws, eigene Darstellung.

\subsection{Analyse der aws Portfolio-Monitoringdaten}

Die aws hatte im Jahr 2012 mittels eines Fragebogens bei 82 im Portfolio befindlichen PreSeed und Seedfinancing geförderten Unternehmen quantitative Angaben zur Unternehmensentwicklung (Beschäftigung, Umsatz, Export, Förderungen, Akquise von zusätzlichem privatem Risikokapital) erhoben. Die befragten Unternehmen trugen die jeweiligen Informationen getrennt für die Jahre 2007 bis 2011 in ein Spreadsheet ein, welche im Folgenden genauer betrachtet werden. Für 65 von 83 Unternehmen lagen innerhalb dieses Betrachtungszeitraums Informationen vor ob sie eine PreSeed bzw. Seedfinancing Förderung erhalten haben. Das entspricht bei einem Gesamtunternehmensbestand an geförderten Seed und PreSeed Unternehmen zwischen 2007 und 2013 von 230 einer Abdeckung von 28\%.

\section{Abbildung 32: Verteilung Grundgesamtheit / aws-Monitoring}
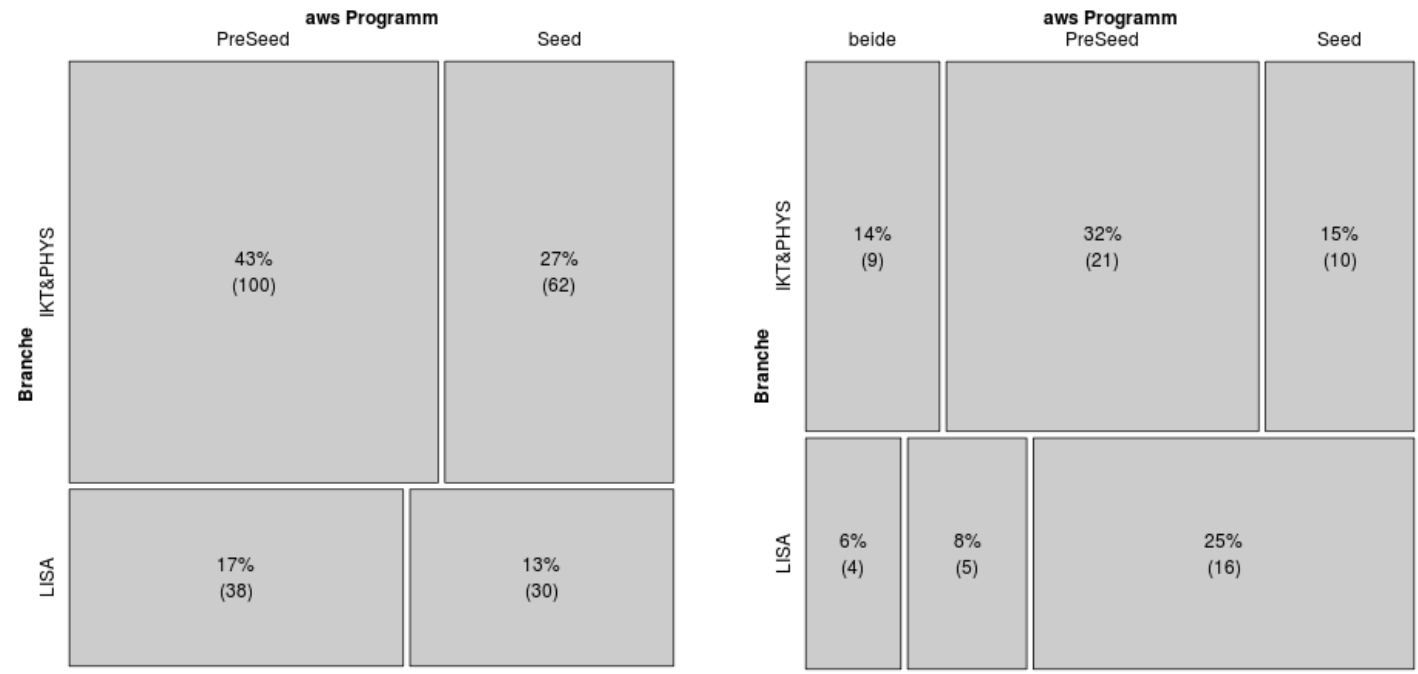

Quelle: aws, eigene Darstellung. Anmerkung: Grundgesamtheit (2007-2013) N=230, Case Studies $N=65$.

Abbildung 32 zeigt die Verteilung der Grundgesamtheit der aws-geförderten Projekte (links) und der Stichprobe der Unternehmensbefragung (rechts). Es zeigt sich, dass hinsichtlich des programmatischen Schwerpunktes (IKT \& Physical Science bzw. LISA) und des Programms (PreSeed bzw. Seedfinancing), eine 
ähnliche Verteilung vorliegt. Seedfinancing Projekte im LISA Bereich sind mit 31\% im Sample etwas überrepräsentiert (Grundgesamtheit 13\%).

\section{Beschäftigung}

Eine der wichtigsten übergeordneten wirtschaftspolitischen Zielsetzungen der Investitionsförderung ${ }^{13}$ ist die Schaffung von Beschäftigung. Abbildung 33 zeigt den durchschnittlichen Beschäftigungsstand 2011 der Unternehmen des Monitorings nach Gründungskohorte. Da das Unternehmen Intercell einen klaren Ausreißer darstellt, wird es farblich getrennt ausgewiesen. Erwartungsgemäß steigt mit steigendem Unternehmensalter der durchschnittliche Beschäftigungsstand. So weisen Unternehmen welche zwischen 2007 und 2009 gegründet wurden im Jahr 2011 einen durchschnittlichen Beschäftigungstand von 8 auf. Unternehmen der drei Jahre älteren Kohorte (2004-2006) weisen bereits doppelt so viele Beschäftigte im Durchschnitt auf. Das durchschnittliche Unternehmen der ältesten Kohorte (1997-2003) bringt es schließlich auf 27 Beschäftigte im Schnitt.

\section{Abbildung 33: Beschäftigungsstand 2011 nach Gründungskohorte}

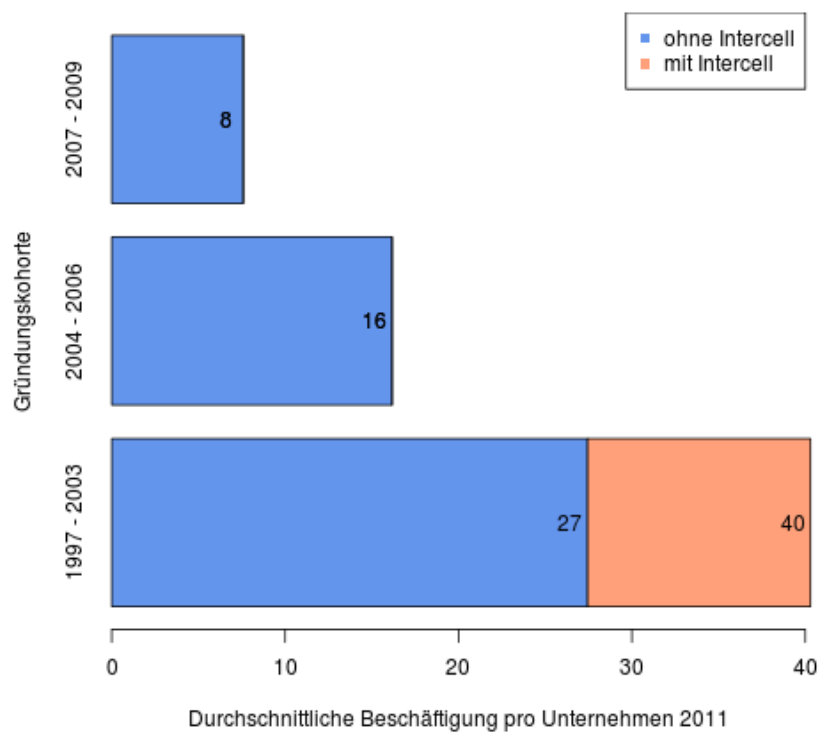

Quelle: aws, eigene Darstellung.

Abbildung 34 verdeutlicht hierzu den Zusammenhang zwischen Unternehmensalter und Beschäftigungswachstum. Auf der x-Achse ist das Gründungsjahr und auf der y-Achse das durchschnittliche Beschäftigungswachstum zwischen 2008-2011 aufgetragen. Das Sample schrumpft durch diese Zeitraumwahl auf 36 Unternehmen. Jüngere Unternehmen wachsen stärker, da diese von geringerem Niveau aus wachsen. Die mittlere Hälfte der Unternehmen (1. bis 3. Quantil) wächst zwischen 7 und 27\% pro Jahr, wobei der Median bei $20 \%$ liegt. Laut einer aktuellen Studie liegt das durchschnittliche Beschäftigungswachstum von jungen (<5 Jahre) Unternehmen in Österreich zwischen 2001 und 2011 bei etwa $2 \%$ pro Jahr. ${ }^{14}$ Dieser Wert kann aufgrund des unterschiedlichen Zeitraums und anderer Berechnungsmethoden der Wachstumsraten nicht direkt verglichen werden. Doch zeigt sich aber ein hohes Beschäftigungswachstum der geförderten Unternehmen innerhalb des JITU-Programms.

\footnotetext{
13 Siehe Knoll (2013).

${ }^{14}$ Siehe Criscuolo et al. (2014).
} 
Abbildung 34: Durchschnittliches Beschäftigungswachstum 2008-2011 nach Gründungsjahr

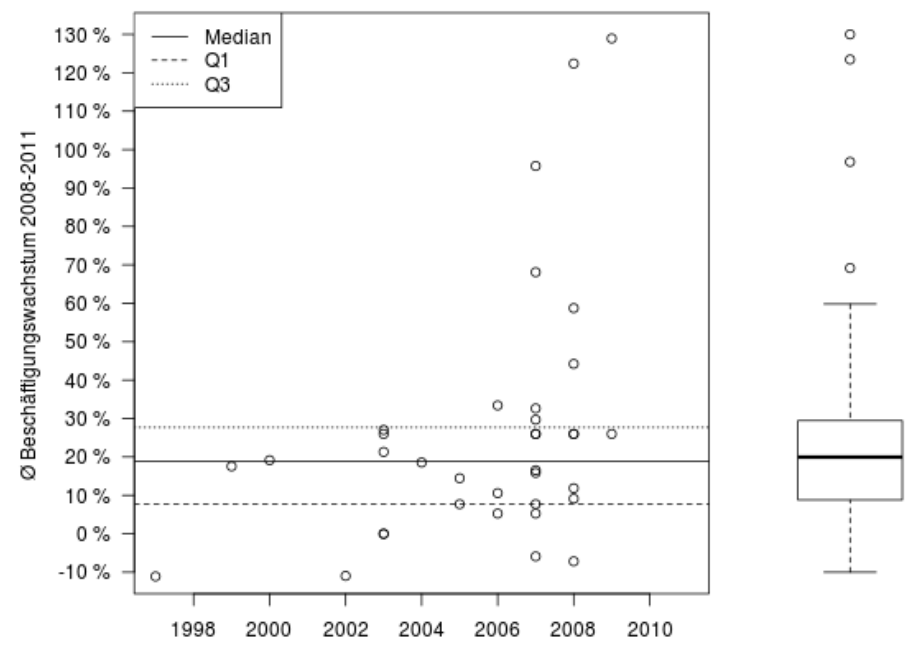

Quelle: aws, eigene Darstellung. $N=36$

Vernetzung in der Finanzierung

Die 82 Unternehmen wurden auch gebeten alle anderen erhaltenen Förderungen bzw. Finanzierungsformen mit entsprechender Summe bekannt zu geben. Dabei wurden die gewichtigen großen Förderschienen wie bspw. der erp-Kredit, das FFG Basisprogramm bzw. AplusB gesondert und andere Programme unter „diverses“ zusammengefasst. Abbildung 35 zeigt die als Kreise alle bezogenen Förderprogramme aller Unternehmen. Um das Sample möglichst groß zu halten, inkludiert dies auch Unternehmen welche im Zeitraum 2007-2011 keine PreSeed bzw. Seedförderung bekannt gegeben haben, da sie dies vor diesem Zeitraum erhalten hatten. Dadurch kann es vorkommen, dass auch starke Verbindungen zwischen Finanzierungsquellen außerhalb des PreSeed und Seedfinancing Programms bestehen können.

Abbildung 35: Vernetzung der Finanzierung - PreSeed und Seed (LISA und IKT \& Physical Science) EKGârantie

ERPRriedite

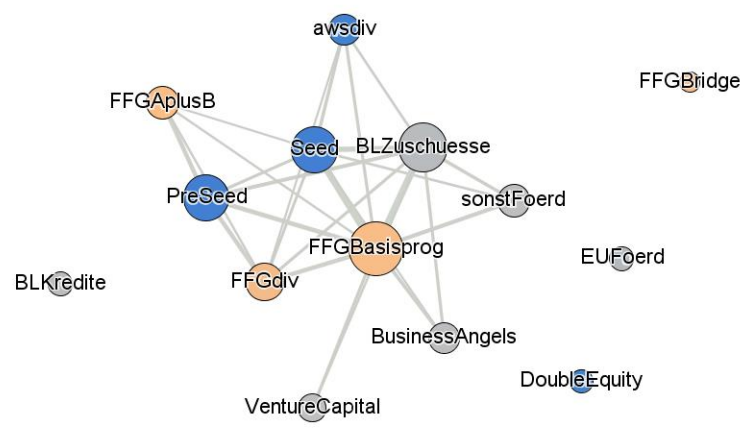

Protrans

BLFdiv

Quelle: aws, eigene Darstellung.

Die Größe des Kreises ist dabei jeweils proportional zur Häufigkeit der Nennung insgesamt. Die gemeinsamen Nennungen zweier Förderprogramme sind mittels der Verbindungen dargestellt. Die 
Förderprogramm PreSeed und Seedfinancing sind stark im Kontext des FFG Basisprogrammes bzw. des FFG AplusB Programms eingebunden. Weitere starke Verbindungen bestehen zu Bundesland Zuschüssen bzw. hinsichtlich des Seedfinancing Programms zu Business Angel Beteiligungen.

Abbildung 36 zeigt das Netzwerk Diagramm für den Programmtyp Life Science. Hier zeigt sich, dass VCGeber und Business Angels deutlich an Gewicht bekommen und ins Zentrum des Netzwerks rutschen. PreSeed und Seedfinancing bereiten hier den Weg zum FFG Basisprogramm und private Risikokapitalgeber.

Abbildung 36: Vernetzung der Finanzierung - LISA (PreSeed und Seed)

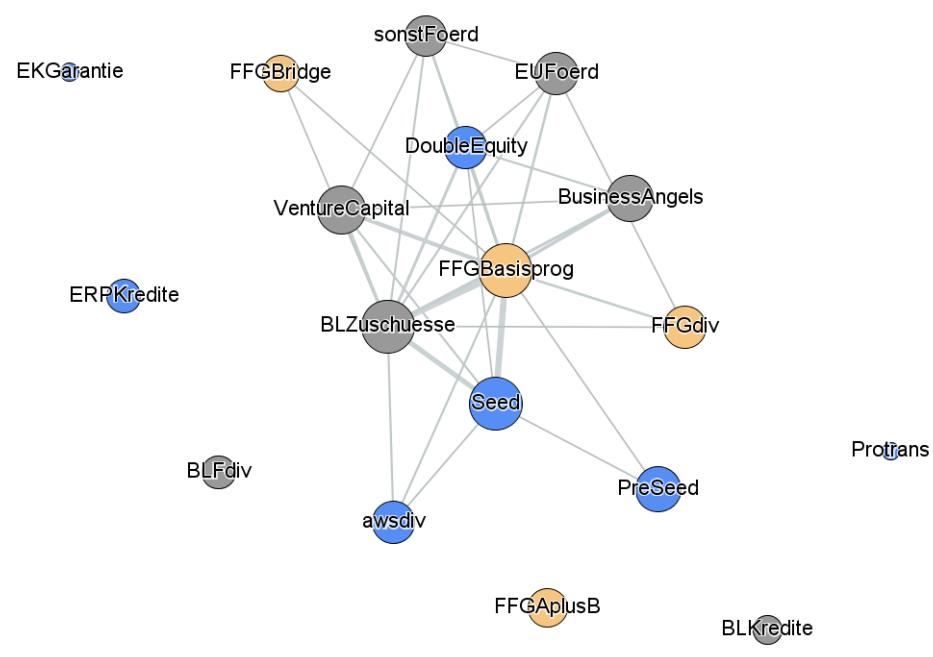

Quelle: aws, eigene Darstellung.

Abbildung 37 zeigt die Vernetzung der Finanzierung im IKT \& Physical Science Segment. Hier stehen PreSeed und Seedfinancing stärker im Zentrum der Finanzierung und privates Risikokapital nimmt an Bedeutung ab.

Abbildung 37: Vernetzung der Finanzierung - IKT\&PHS (PreSeed und Seed)

EUFoerd

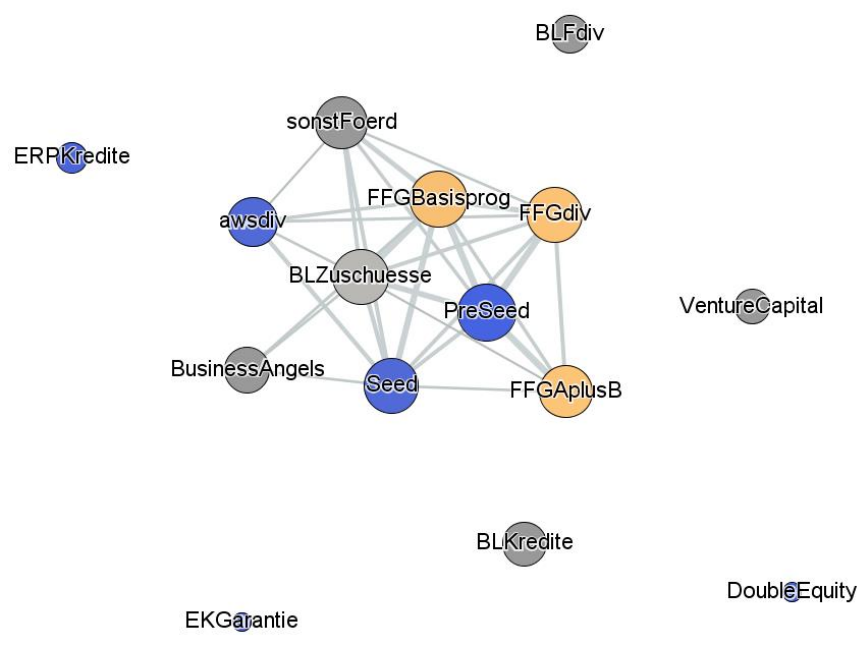

Quelle: aws, eigene Darstellung.

Um die Relevanz der einzelnen Finanzierungsquellen für die Programmschwerpunkte stärker herauszuarbeiten, werden in Abbildung $\mathbf{3 8}$ die Finanzierungsquellen aller befragten Unternehmen mit einem Gründungsjahr von mindestens 2007 dargestellt. Der Grund für diese Einschränkung liegt darin, dass 
hier die Bedeutung der Förderungen für junge, finanzierungsbeschränkte Unternehmen herausgearbeitet werden soll. Im Diagramm enthalten sind die Summen in Euro der jeweiligen Unternehmen über den Gesamten Zeitraum 2007-2011.

\section{Abbildung 38: Finanzierungsquellen der geförderten Unternehmen}

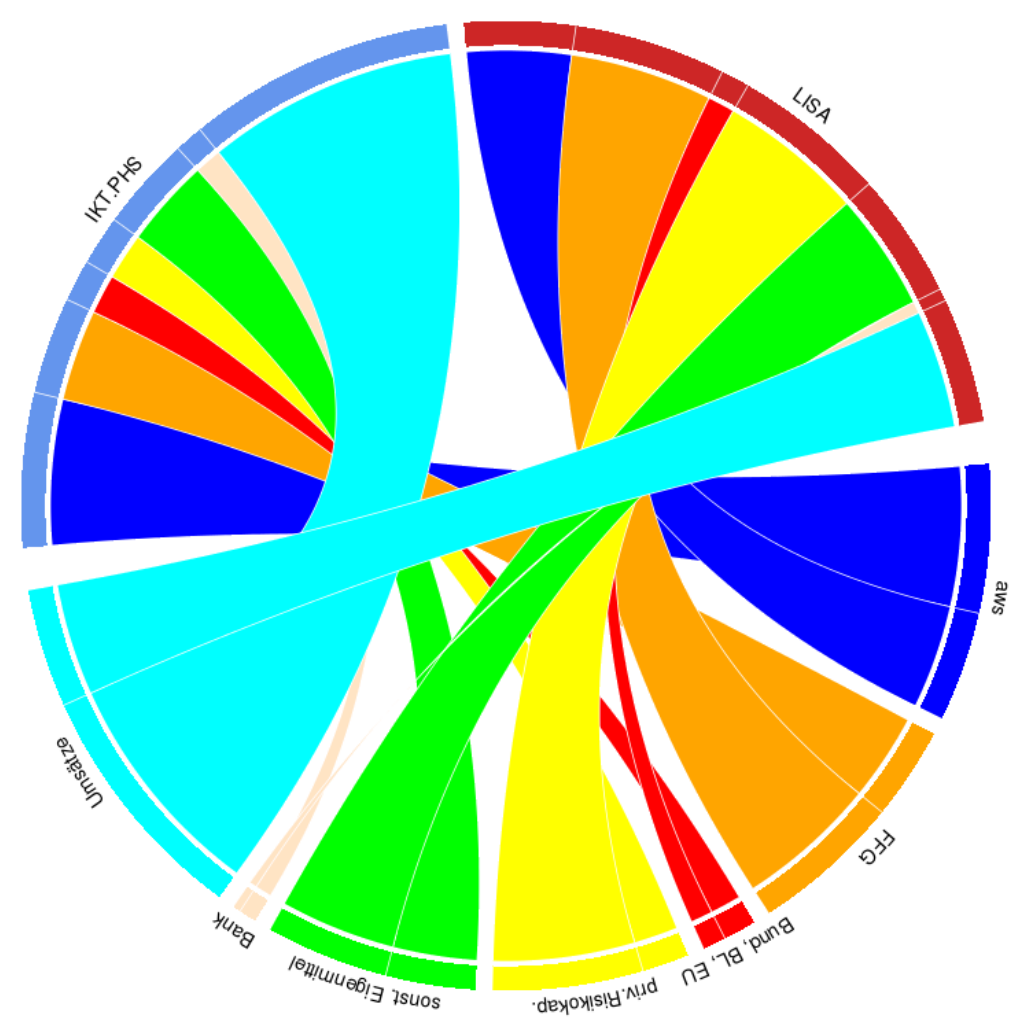

Quelle: aws, eigene Darstellung. Anmerkung: Im Sample sind nur Unternehmen des Monitoring mit einem Gründungsjahr von frühestens 2007. [n=58 Unternehmen]

Es zeigt sich, dass Unternehmen mit Schwerpunkt IKT \& Physical Science bereits in jungem Alter stark über Umsätze (welche als Approximation für den Cash-Flow dienen) finanzieren. Förderungen der aws nehmen hier einen etwas stärkeren Anteil an der Gesamtfinanzierung an als bei den Unternehmen aus dem Life Science Bereich. Dort hingegen wird schon in jungen Jahren verstärkt privates Risikokapital (Venture Capital, Business Angels und stille Beteiligungen) akquiriert. Zuletzt zeigt sich im Vergleich der Finanzierungshöhe (im Gegensatz zur vorher in den Netzwerkdiagrammen dargestellten Ko-Nennungen) auch die geringe Rolle der Förderungen der Gebietskörperschaften und der Bank. Sonstige Eigenmittel umfassen Privateinlagen der Gründer sowie sonstige nicht näher spezifizierte Eigenmittel. Tabelle 6 zeigt die dahinterliegenden Daten. 
Tabelle 6: Finanzierungsquellen in Prozent und absolut

\begin{tabular}{|c|c|c|c|c|}
\hline \multirow{2}{*}{ Umsätze } & & LISA & IKT\&PHS & beide \\
\hline & & $18 \%$ & $37 \%$ & $28 \%$ \\
\hline aws & & $16 \%$ & $21 \%$ & $19 \%$ \\
\hline FFG & & $21 \%$ & $13 \%$ & $17 \%$ \\
\hline Eigenmittel und son & tige & $18 \%$ & $13 \%$ & $15 \%$ \\
\hline privates Risikokapit & & $22 \%$ & $7 \%$ & $13 \%$ \\
\hline Bund, Länder, EU & & $4 \%$ & $6 \%$ & $5 \%$ \\
\hline \multirow[t]{2}{*}{ Bank } & & $2 \%$ & $4 \%$ & $3 \%$ \\
\hline & IKT\&PHS & & LISA & beide \\
\hline Umsätze & 26.700 .435 & & .809 .822 & 36.510 .257 \\
\hline aws & 15.407 .158 & & 685.410 & 24.092 .569 \\
\hline FFG & 9.665 .164 & & .866 .686 & 21.531 .851 \\
\hline sonst. Eigenmittel & 9.400 .169 & & .816 .396 & 19.216 .565 \\
\hline priv.Risikokap. & 4.947 .878 & & .073 .519 & 17.021.397 \\
\hline Bund, BL, EU & 4.165 .763 & & .244 .780 & 6.410 .543 \\
\hline Bank & 2.841 .985 & & 980.000 & 3.821 .985 \\
\hline
\end{tabular}

Quelle: aws, eigene Darstellung. Anmerkung: Im Sample sind nur Unternehmen des Monitoring mit einem Gründungsjahr von frühestens 2007. [n=58 Unternehmen]

In Abbildung 39 (bzw. in Zahlen Tabelle 7) sind die Streuungen des Anteils aller erhaltener Förderungen an der Gesamtfinanzierung und des Eigenkapital-Hebels dargestellt. Der Eigenkapitalhebel definiert sich als Verhältnis zwischen Eigenkapital zu Förderungen, d.h. wieviel Euro Eigenmittel werden von den Unternehmen pro Euro Förderung eingesetzt. Unternehmen mit einem Gründungsjahr von zwischen 20072011 weisen im Schnitt einen Förderanteil von zwischen 55 und 87\% (1. bzw. 3. Quantil) auf. Der Eigenkapital-Hebel schwankt zwischen 0 und 5,5 Euro pro Fördereuro, wobei der mittlere (Median-) Unternehmer etwa 33 Cent pro Fördereuro beisteuert. Der niedrige EK-Hebel ergibt sich daraus, dass lediglich sehr junge Unternehmen im Sample inkludiert sind, da nur für diese Unternehmen Angaben über die Förderungssummen verfügbar sind. Eine zeitlich-verknüpfbare Fortführung des Monitorings würde es hier in Zukunft ermöglichen aussagekräftigere (insbesondere auf Basis von Gründungskohorten) Aussagen bezüglich des Eigenkapitalhebels zuzulassen.

\section{Abbildung 39: Förderanteil an der Gesamtfinanzierung und Eigenkapital-Hebel}

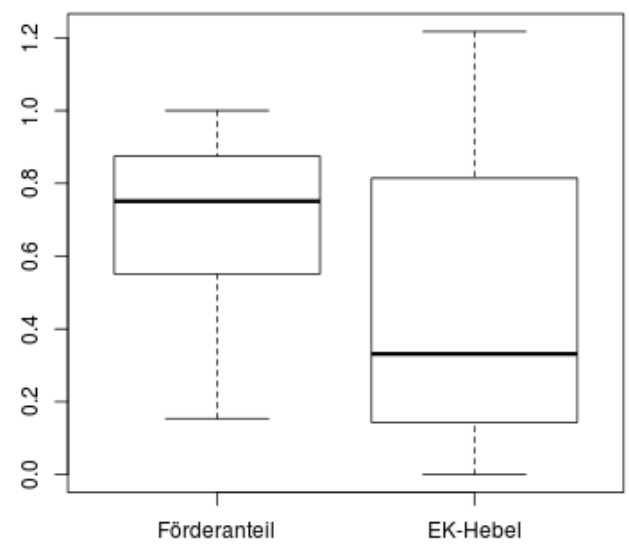

Quelle: aws, eigene Darstellung. Anmerkung: Im Sample sind nur Unternehmen des Monitoring mit einem Gründungsjahr von frühestens 2007. $n=58$ Unternehmen -Outlier [7 bei EK-Hebel] nicht dargestellt 
Tabelle 7: Förderanteil an der Gesamtfinanzierung und EK-Hebel, Verteilung

\begin{tabular}{lrrrrrr} 
& Min. & erstes Quantil & Median & Durchschnitt & drittes Quantil & Max. \\
\hline Förderanteil & $15 \%$ & $55 \%$ & $75 \%$ & $70 \%$ & $87 \%$ & $100 \%$ \\
EK-Hebel & 0,00 & 0,14 & 0,33 & 0,75 & 0,81 & 5,50 \\
\hline
\end{tabular}

Quelle: aws, eigene Darstellung. Anmerkung: Im Sample sind nur Unternehmen des Monitoring mit einem Gründungsjahr von frühestens 2007. $n=58$ Unternehmen

Die Box in Abbildung 40 zeigt für die Unternehmen nach Gründungskohorten ob eine Venture Capital Finanzierung vorliegt. Bei den 1997 bis 2007 gegründeten Unternehmen (28 Fälle) haben 10 Fälle VCFinanzierung. Der VC-Status unterscheidet bezüglich des Alters der Unternehmen statistisch-signifikant, wobei ältere Unternehmen zu höheren Anteilen eine VC-Finanzierung aufweisen. Hingegen weist kein Unternehmen der jüngsten Kohorte (2010 und 2011) eine VC-Finanzierung auf. Von den 28 Unternehmen der mittleren Gründungskohorte lag bei 5 Unternehmen eine VC-Finanzierung vor.

\section{Abbildung 40: Venture Capital Finanzierung nach Gründungskohorten}

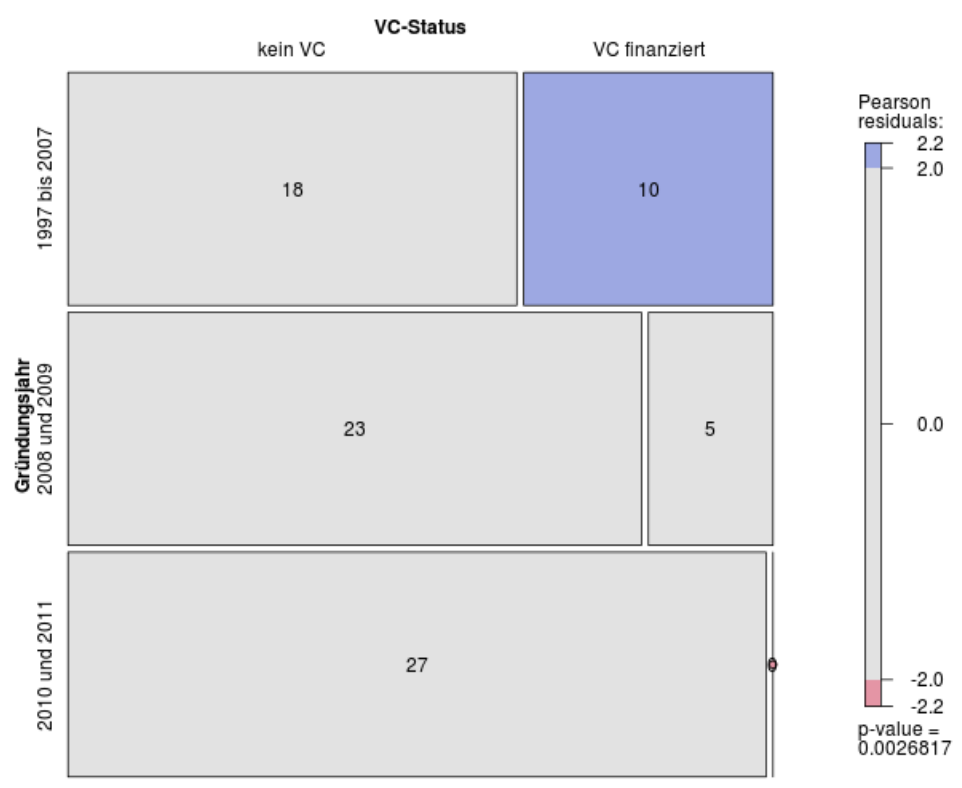

Quelle: aws, eigene Darstellung. $n=83$

Abbildung 41 stellt analog dazu das Auftreten von Business Angel über die Gründungskohorten dar. Auch hier zeigt sich ein stetiger Anstieg mit zunehmenden Unternehmensalter, jedoch liegen keine statistischsignifikanten Unterschiede vor. Business Angel steigen schon bei jüngeren Unternehmen verstärkt ein, da hier zum Teil schon in der Konzipierung des Geschäftsmodells operativ oder strategisch Unterstützung für den Gründer stattfinden kann („Hands-On“ Business Angel). Abbildung 42 und Abbildung 43 zeigen das Auftreten von F\&E-Ausgaben, Patenten bzw. das Vorliegen von Umsätzen über die Gründungskohorten. Erwartungsgemäß steigt die Häufigkeit der Unternehmen mit Umsätzen bzw. Patenten mit steigendem Alter. Hinsichtlich der F\&E-Ausgaben zeigt sich, dass aufgrund des Hochtechnologie-Charakters des JITUProgramms bereits bei Unternehmen in jungen Jahren schon F\&E-Ausgaben mit hoher Wahrscheinlichkeit auftreten. 
Abbildung 41: Business Angel Finanzierung nach Gründungskohorten

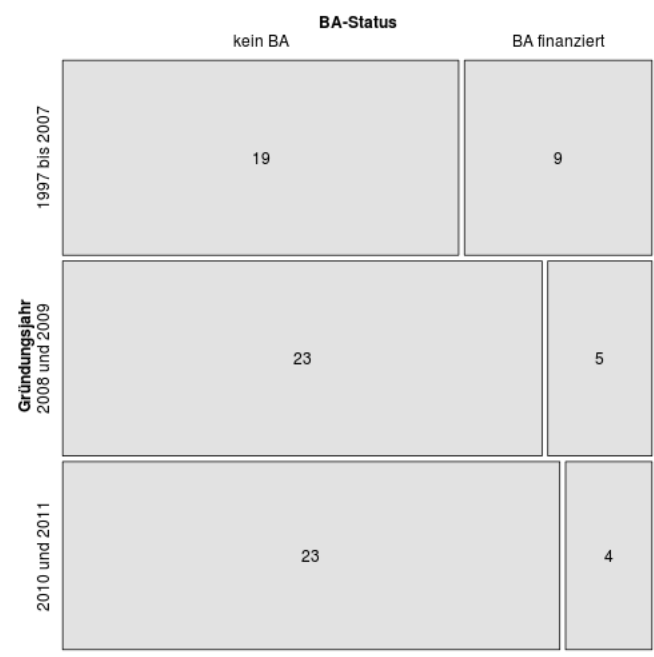

Quelle: aws, eigene Darstellung. $n=83$

Abbildung 42: Patente und F\&E-Ausgaben nach Gründungskohorten
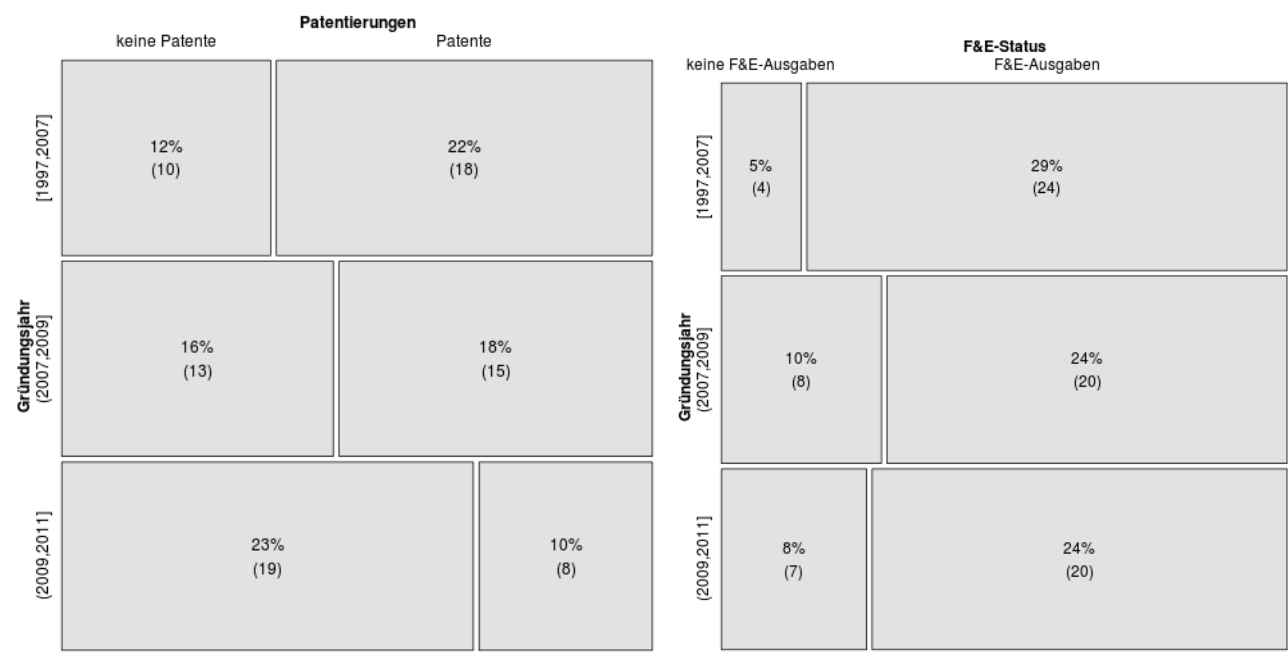

Quelle: aws, eigene Darstellung. $n=83$ 


\section{Abbildung 43: Umsätze nach Gründungskohorten}

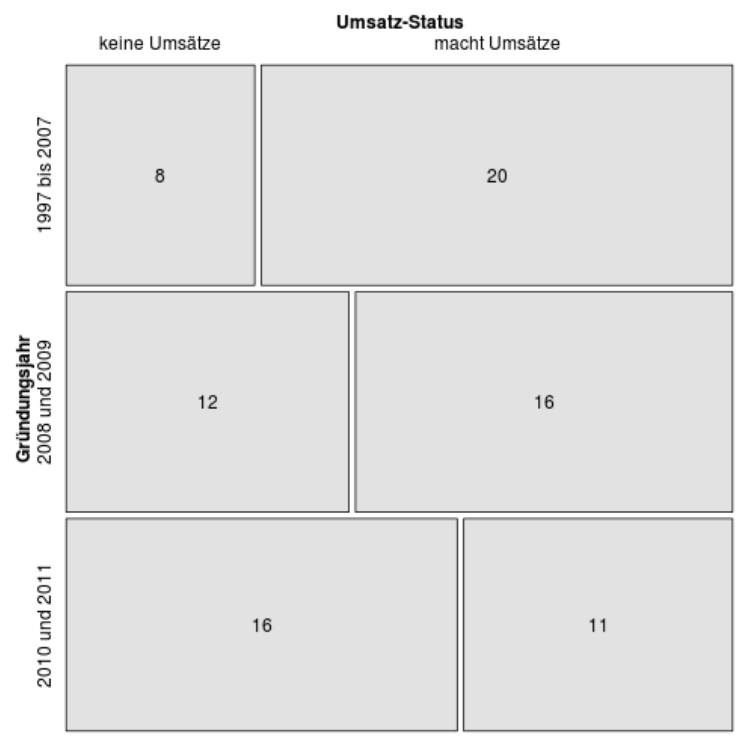

Quelle: aws, eigene Darstellung. $n=83$

\subsection{Management auf Zeit (MaZ)}

Das Programm Management auf Zeit wurde mit der JITU Richtlinie 2007 etabliert und ging Ende 2008 mit dem ersten beschlossenen Projekt in Operation. Der zeitliche Verlauf der Anfragen, Projektbeschlüsse und Förderzusagen bzw. -auszahlungen ist in Tabelle 8 dargestellt. Nachdem der Nachfrage-Stau der betreuten und damit qualifizierten Seedfinancing-Projekte im Jahr 2009 mit 10 bewilligten Projekten abgebaut wurde, pendelte sich die Anzahl der Anfragen zwischen 2 und 5 und der Zusagen zwischen 1 und 5 ein. Seit Programmstart wurden 21 Zusagen in Höhe von insgesamt etwa 750.000 Euro erteilt. Davon wurden bisher etwa 480.000 Euro ausbezahlt. Hinsichtlich der Anfragen ist anzumerken, dass zusätzlich zu den offiziellen Anfragen viele inoffizielle Anfragen betreuter Seedfinancing Unternehmen eintreffen.

Tabelle 8: Entwicklung Management auf Zeit, 2008-2013

\begin{tabular}{crrrr} 
Jahr & Anfragen & beschlossene Projekte & Förderzusage in EUR & ausbezahlt \\
\hline 2008 & 1 & 1 & 12.250 & - \\
2009 & 13 & 10 & 325.750 & 80.000 \\
2010 & 5 & 5 & 237.000 & 186.450 \\
2011 & 2 & 1 & 46.800 & 101.950 \\
2012 & 2 & 3 & 82.250 & 65.018 \\
2013 & 3 & 1 & 50.000 & 45.825 \\
\hline Summe & 26 & 21 & 754.050 & 479.243 \\
\hline
\end{tabular}

Quelle: aws, eigene Darstellung.

Tabelle 9 zeigt eine Zusammenfassung der Kennzahlen des MaZ Programms. Dabei wurden die Summen über den gesamten Zeitraum betrachtet, da in einzelnen Jahren nur geringe Fallzahlen auftreten. Die Annahmequote liegt insgesamt bei $80 \%$, bei einer durchschnittlichen Förderhöhe von etwa 36.000. Setzt man die zugesagten MaZ Projekte mit den zugesagten Seedfinancing Projekten in Relation, so wird etwa jedes vierte Seedfinancing Projekt durch MaZ begleitet. 
Tabelle 9: Kennzahlen Management auf Zeit, 2008-2013

Summe $2008-2013$

\begin{tabular}{lr}
\hline Annahmequote & $80,8 \%$ \\
durchschnittliche Förderhöhe & 35.907 \\
zugesagte Seed Projekte & 92 \\
MaZ / Seed Projekte & $23 \%$ \\
\hline
\end{tabular}

Quelle: aws, eigene Darstellung.

Wiederum wurden zusätzlich zu den aws-Daten qualitative Interviews bei Seedfinancing-geförderten Unternehmen, die auch Management auf Zeit in Anspruch nahmen durchgeführt. Die (nicht repräsentativen) Ergebnisse zeigen die Ambivalenz externer Beratung. Einerseits wird die Hereinnahme von externem Knowhow als wichtiger Input geschätzt und die Arbeitsbelastung für die Gründer wird deutlich reduziert, andererseits wird angemerkt, dass zeitlich befristete externe Berater die Geschäftsidee nicht so „verinnerlicht" haben wie die Gründer („fehlender Kontakt zum Produkt und Kunden“). In einem anderen Fall wurden aber durchaus von positiven Erfahrungen berichtet und der externe Berater war entscheidenden für den Aufbau von Vertriebsstrukturen und den Einstieg in den Export. In diesem Fall ging es sogar so weit, dass nach Ablauf der Förderperiode der externe Berater über einen Vertrag auf Provisionsbasis mit dem Unternehmen verbunden blieb. Grundsätzlich positiv wurde die rasche und unbürokratische Abwicklung der Förderung betrachtet. 


\section{6. i2 - Business Angels}

Das Programm i2 - Business Angels besteht seit 1997 und wurde seither dreimal evaluiert. ${ }^{15}$ Abbildung 44 zeigt den Verlauf der Anfragen und Listungen der Unternehmen beginnend mit 1997. Die dahinterliegenden Daten können Tabelle 21 im Anhang entnommen werden. Die Anfragen der Unternehmen erreichten erst mit 2000 eine für einen Matching-Prozess zwischen Unternehmen und Business Angels kritische Menge etwa 250. Danach entwickelten sich die Anfragen etwas volatil um etwa 350 pro Jahr und lagen zuletzt im Jahr 2013 bei 457. Die Anzahl der für die Business Angel gelisteten Unternehmen stieg bis 2000 auf etwa 100 an und fiel dann stetig bis 2010 auf 30 ab. In den letzten drei Jahren werden jeweils um die 60 Unternehmen gelistet, was dem Zielwert der Programmverantwortlichen entspricht.

Abbildung 44: Unternehmensanfragen und -listungen, 1997-2013
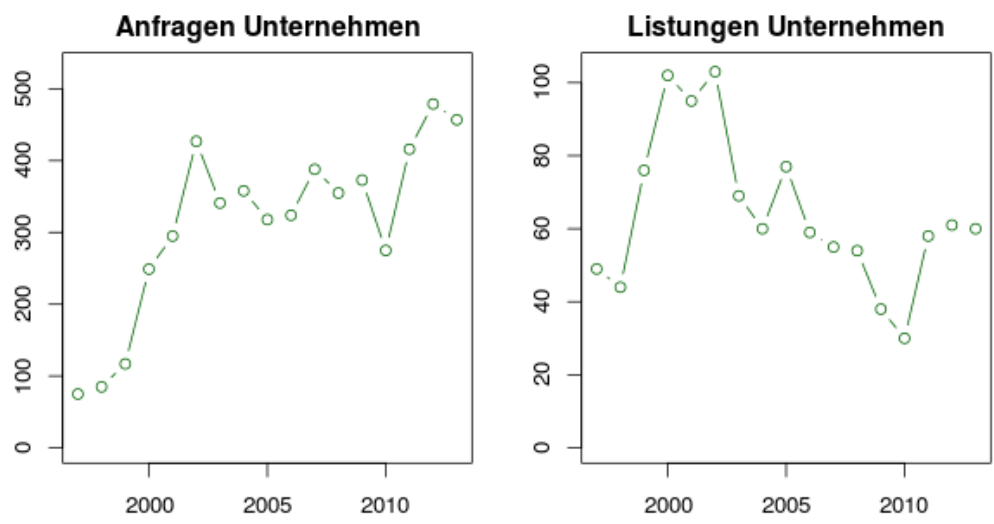

Quelle: aws, eigene Darstellung.

Abbildung 45 zeigt den zeitlichen Verlauf der gelisteten Business Angel, sowie die Anzahl der erfolgreichen Vermittlungen. Das Netzwerk begann 1997 mit 37 Business Angels und überschritt im Jahr 2006 die 100 und stagnierte bis 2010 auf etwa 120. Die Überschreitung der 150er Marke durch das rasante Wachstum nach 2010 führte letztlich zu starken Netzwerkeffekten und einer höheren Anzahl an Vermittlungen. Diese wuchsen von zwischen 1 und 7 bis 2010 auf durchschnittliche 12 pro Jahr zwischen 2010 und 2013 an.

Abbildung 45: Business Angel Listungen und Vermittlungen, 1997-2013
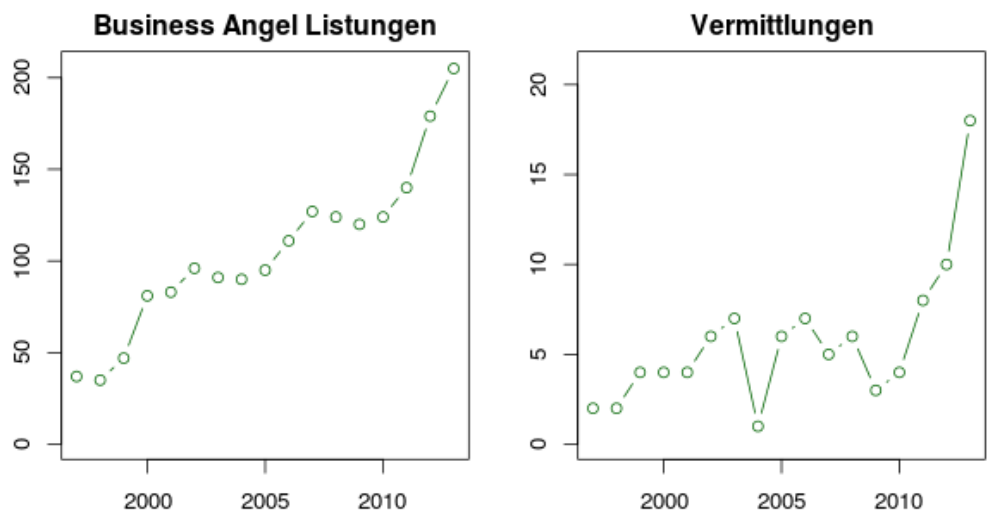

Quelle: aws, eigene Darstellung.

\footnotetext{
${ }^{15}$ Siehe Jörg, Mahlich und Ohler (2002), Jörg et al. (2006) und Gassler et al. (2011).
} 
Wie in Abbildung $\mathbf{4 7}$ gesehen werden kann, ist die Selektivität der Unternehmenslistungen im Zeitverlauf gestiegen (fallender Anteil an Listungen bezogen auf die Projektanfragen) und die Vermittlungsquote durch die höhere Anzahl an Business Angel und die stärkere Selektion der Projekte gestiegen.

Abbildung 46: i2 Listungs- und Vermittlungsquote, 1997-2013
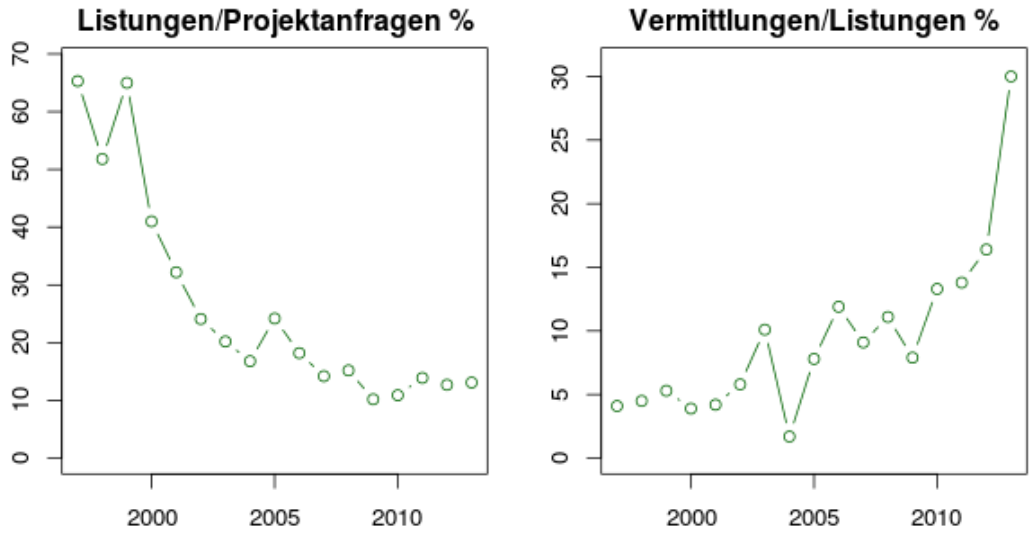

Quelle: aws, eigene Darstellung.

Abbildung 47 zeigt die Gesamtbeteiligungen der Business Angel sowie die durchschnittliche Beteiligung pro Vermittlung des Netzwerks im Zeitverlauf. Durch das hohe Ausmaß an Heterogenität innerhalb der Business Angel (Branche, Phase, Strategie, Exitplan) und der Investitionsvorhaben schwanken diese Werte naturgemäß stark. Spezifische Großprojekte in vereinzelten Jahren führen zu starken Ausreißern. Über den gesamten Programmverlauf wurden insgesamt 12,7 Mio. Euro von BA an insgesamt 97 Unternehmen vermittelt.

Abbildung 47: Beteiligungen gesamt und pro Vermittlung, 1997-2013
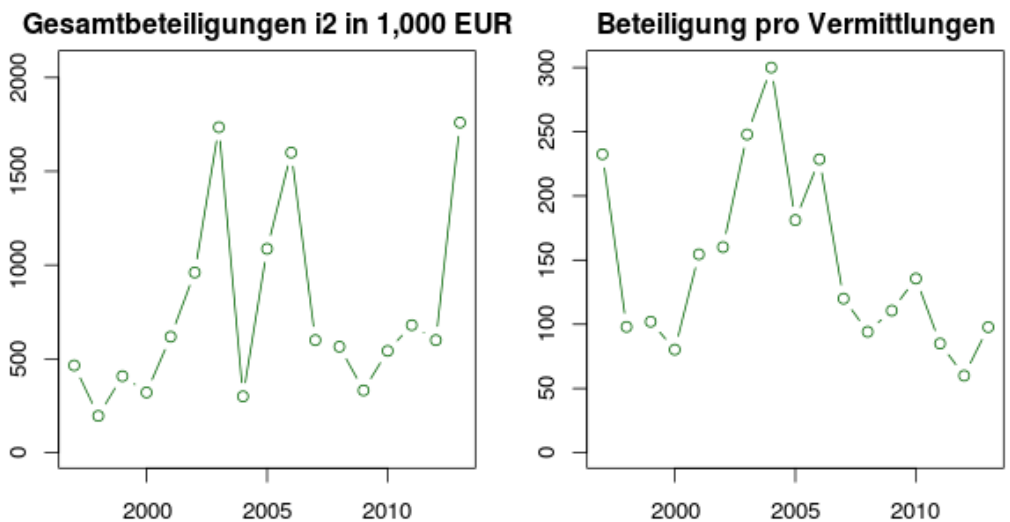

Quelle: aws, eigene Darstellung.

Im Schnitt betrug eine Vermittlung etwa 130.000 Euro (siehe Tabelle 10) mit einem Minimum von 60.000 und einem maximalen Beteiligungswert pro Vermittlung von 300.000 Euro. Im Vergleich dazu lag das durchschnittliche BA Investment pro Deal in den USA im Jahr 2013 bei 350.800 USD (264.159 Euro zu Wechselkurs 2013) bzw. im Jahr 2012341.800 USD (266.034 Euro zu Wechselkurs 2012) ${ }^{16}$. In Europa betrug das durchschnittliche BA-Investment pro Unternehmen im Jahr $2012^{17} 174.800$ Euro.

\footnotetext{
${ }^{16}$ Siehe Sohl (2014) bzw. Sohl (2013).

${ }^{17}$ Siehe EBAN (2013).
} 
Tabelle 10: i2 Business Angel Investment pro Deal, 1997-2013

\begin{tabular}{|c|c|c|c|}
\hline Jahr & i2 Vermittlungen & i2 Beteiligung & Beteiligung pro Vermittlung \\
\hline 1997 & 2 & 465.000 & 232.500 \\
\hline 1998 & 2 & 196.000 & 98.000 \\
\hline 1999 & 4 & 408.000 & 102.000 \\
\hline 2000 & 4 & 321.000 & 80.250 \\
\hline 2001 & 4 & 618.000 & 154.500 \\
\hline 2002 & 6 & 960.700 & 160.117 \\
\hline 2003 & 7 & 1.735 .000 & 247.857 \\
\hline 2004 & 1 & 300.000 & 300.000 \\
\hline 2005 & 6 & 1.087 .000 & 181.167 \\
\hline 2006 & 7 & 1.600 .000 & 228.571 \\
\hline 2007 & 5 & 600.000 & 120.000 \\
\hline 2008 & 6 & 565.000 & 94.167 \\
\hline 2009 & 3 & 332.000 & 110.667 \\
\hline 2010 & 4 & 542.500 & 135.625 \\
\hline 2011 & 8 & 680.000 & 85.000 \\
\hline 2012 & 10 & 600.000 & 60.000 \\
\hline 2013 & 18 & 1.760 .000 & 97.778 \\
\hline Summe & 97 & 12.770 .200 & 131.652 \\
\hline
\end{tabular}

Quelle: aws, eigene Darstellung.

Abbildung 48 zeigt die regionale Verteilung der i2 Unternehmenslistungen für die Jahre 2011 bis 2013. Ähnlich der Verteilung der PreSeed und Seedfinancing Anfragen bzw. Zusagen, dominieren Wien und die Steiermark mit in Summe etwa der Hälfte der Listungen. Geht man davon aus, dass die meisten Business Angel ihren Arbeitsschwerpunkt in Wien haben und dass Unternehmen die regionale Nähe zum Investor suchen, erklärt sich auch der hohe Anteil an niederösterreichischen Listungen. Ein mit 5\% nicht unbeträchtlicher Teil an Listungen entfällt auf Unternehmen aus dem Ausland. Dies betrifft Unternehmen aus den benachbarten Ländern Deutschland, Italien, der Schweiz und Slowenien.

\section{Abbildung 48: Regionale Verteilung der i2-Listungen, 2011-2013}

i2 Listungen, 2011-2013

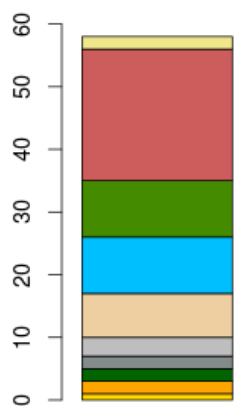

\begin{tabular}{|c|c|c|c|}
\hline 2011 & & 2012 & 201 \\
\hline $\begin{array}{l}=\text { VBG } \\
=\text { BGLD } \\
=\text { KTN }\end{array}$ & $\begin{array}{l}=O O= \\
=S B G \\
=T\end{array}$ & $\begin{array}{l}=\text { NÖ } \\
=\text { STMK } \\
=\mathrm{W}\end{array}$ & " Ausland \\
\hline
\end{tabular}

Regionale Verteilung, Summe 2011-2013

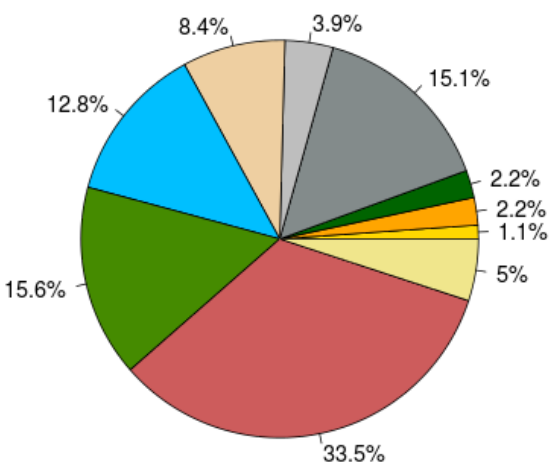

Quelle: aws, eigene Darstellung.

Abbildung 49 zeigt die Verteilung der i2 Portfoliounternehmen zum Zeitpunkt 2012 nach Branche. Die meisten Unternehmen sind im Cleantech, Chemie bzw. im IKT/Mobile Bereich tätig. Im Life Science / Pharmabereich finden sich wiederrum nur 7\% der Unternehmen. 
Abbildung 49: Branchenverteilung der i2 Portfoliounternehmen, 2012

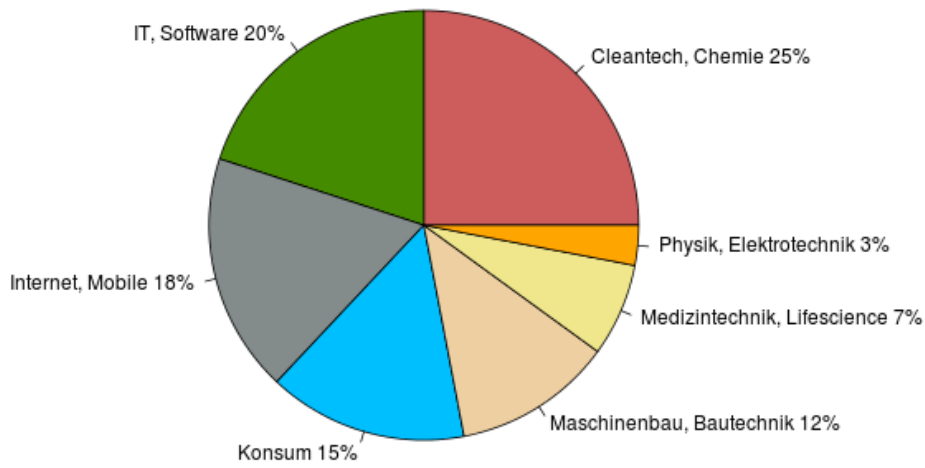

Quelle: aws, eigene Darstellung.

Ergänzend zu den Auswertungen der aws-Daten wurden wiederum qualitative Interviews durchgeführt und zwar sowohl bei Unternehmen, die im Zuge von 12 erfolgreich an Business Angels vermittelt wurden, als auch bei Business Angels, die durch 12 an entsprechend für sie interessante Investitionsprojekte vermittelt wurden. Die qualitativen Ergebnisse zeigen, dass die Unternehmen neben dem Finanzierungsinput durch das Bereitstellen von Risikokapital auch das Einbringen des unternehmerischen Knowhows von Seiten der Business Angels schätzen. Notwendig ist jedoch eine Vertrauensbeziehung zwischen Unternehmer/Gründer und Business Angel.

Die befragten Business Angels sind durchwegs der Meinung, dass die Vorselektion und die Zurverfügungstellung kurzer Unternehmens-Fact-Sheets von Seiten der aws eine merkliche Erleichterung für den Suchprozess nach interessanten Beteiligungsmöglichkeiten darstellt. Der regelmäßige Erhalt derartiger Fact Sheets wird dabei nicht als Belastung angesehen, d.h. die „Kosten-Nutzen“-Relation wird als sehr gut betrachtet. Hinsichtlich der Investitionsintensität der Business Angels hat sich ergeben, dass sowohl das Engagement von Seiten der Business Angels was die Zahl der Investments betrifft (von einem bis über fünf) als auch was die Intensität der Beziehung zwischen Business Angel und Unternehmen von Fall zu Fall stark variiert (von lediglich Ausübung der Kontrollfunktion in den Gesellschafterversammlungen bis hin zu „beinahe“ operativer Mitarbeit).

Angemerkt wurde in einem Fall, dass die vermehrte Organisation von Business Angels Meetings dazu beitragen könnte, das Netzwerk der österreichischen Business Angels zu verdichten. Diese Meetings sollten dabei durchaus „exklusiver" sein im Sinne, dass mehr Investoren und weniger andere Stakeholder vertreten sein sollten. 


\section{Bewertung der Programmwirkung}

\subsection{JITU-Governance}

Für die Einschätzung der Governance wurden mit den jeweiligen operativen Programmverantwortlichen der aws für JITU und 12 gesonderte Interviews durchgeführt. Zusätzlich wurden in zwei workshop-artigen Projektpräsentationen auch entsprechende Einschätzungen von VertreterInnen des Prinzipals (BMWFW) diskutiert. Daneben wurden auch noch mit „erweiterten“ Stakeholdern (AplusB-Zentren, awsGründerfonds) qualitative Interviews durchgeführt.

Governance von JITU (PreSeed, Seedfinancing, MaZ)

Ein von den Programmverantwortlichen und geförderten Unternehmen adressiertes Problemfeld betrifft die verzögerte Auszahlung von (vorbehaltlich) zugesagten Fördermitteln, die dann auftreten kann wenn während eines Übergangs zwischen zwei 3-Jahres-Verträgen die Beschlussfassung über die Ausführungsverträge nicht zeitgerecht erfolgt. Eine solche Verzögerung kann bei Gründungsprojekten die Gefahr bergen, dass sich das „Window of Opportunity“ (Umsetzung der „richtigen“ Geschäftsidee zum „richtigen“ Zeitpunkt) für das Unternehmen schließt. Dies betrifft vor allem Geschäftsideen im Bereich der IT / Mobile, wo eine möglichst kurze „time-to-market“ unter Umständen von entscheidender Bedeutung sein kann. Allgemein kann eine verzögerte Auszahlung bereits zugesagter Mittel zu Liquiditätsproblemen innerhalb des Unternehmens führen, die den Fortbestand gefährden können, obwohl das Unternehmen grundsätzlich überlebensfähig wäre bzw. mittel- und langfristig hohe Erfolgschancen hat. Letztlich könnte dies zu negativen Imageeffekten für das Branding Österreichs als Standort für High-Tech Gründungen führen, wenn das Flaggschiff-Förderprogramm für High-Tech-Gründungen aus solchen Gründen seinen derzeit international durchaus so wahrgenommenen "Good Practice“-Charakter ${ }^{18}$ verlieren sollte. Die verzögerten Auszahlungen führen weiters zu einem „Projektstau“ bereits zugesagter Projekte, deren Bearbeitung dann bei Zuweisung der Mittel zu aws-internen personellen und zeitlichen Ressourcenproblemen führen kann.

Innerhalb der aws werden derzeit Änderungen hinsichtlich der Entscheidungsgremien angedacht, wobei vor allem eine thematische Aufteilung in Form von zwei Gremien mit jeweiligen Fachleuten aus den Bereichen IKT/Physical Sciences einerseits und Life Sciences andererseits vorgesehen ist. Aus Sicht des Evaluierungsteams wäre eine derartige thematische Aufteilung zu begrüßen, wobei gleichzeitig auch regelmäßige und fixe, sowie nach außen hin (für potentielle Förderwerber) sichtbare Meetings bezüglich Förderentscheidungen festgelegt werden sollten.

Bei Abweichungen von Standardverträgen (z.B. Änderungen Rückzahlungsreihenfolge) ist derzeit die aws nicht entscheidungsautonom. Die entsprechenden Rücksprachen mit dem Prinzipal (BMWFW) stellen sich als zeitintensiv dar und verringern die Abwicklungseffizienz, was negative Auswirkungen auf die Fördernehmer nach sich ziehen kann. Dies betrifft insbesondere die Module PreSeed und MaZ.

Innerhalb der aws wird derzeit auch für das PreSeed Modul diskutiert inwieweit die aws auch an einem möglichen Erfolg (Entwicklung zum High Flyer) partizipieren könnte. Diese Frage stellt sich vor allem dann wenn PreSeed in Zukunft auch für junge „GmbH light“ (im Alter von bis zu 6 Monaten) geöffnet werden sollte.

\footnotetext{
${ }^{18}$ Dieser "Good Practice“-Charakter des Förderprogramms wurde z.B. in Gesprächen mit internationalen VC-Managern betont.
} 
Explizit wird vom Team der aws-Programmverantwortlichen eine intensivere Abstimmung mit dem AplusBProgramm bzw. den AplusB-Zentren erwünscht. Zwar gibt es regelmäßig Treffen und aws-Referenten sitzen vielfach auch in den einschlägigen Gremien von AplusB-Zentren, trotzdem wird die Meinung vertreten, dass diesbezüglich noch Verbesserungspotential bestünde. Dabei ist jedoch zu erwähnten, dass von Seiten der befragten AplusB-Zentren die Synergieeffekte zwischen den JITU-Programmen und AplusB als durchaus hoch eingeschätzt werden und wichtig für das Aufrechterhalten einer konstanten Projekt-Pipeline sind. Betont wurde, dass die Projektpipeline durchaus auch in beide Richtungen möglich ist (d.h. ein bereits gefördertes PreSeed-Projekt wendet sich an ein AplusB-Zentrum. Von Seiten der befragten AplusB-Zentren wurde als eine Möglichkeit der Intensivierung der programmübergreifenden Zusammenarbeit z.B. direkte Besprechungen über Projekte mit den aws-Referenten über die eingereichten Projekte und die Vorabprüfung gemeinsam durchzuführen. Insgesamt sehen sich die AplusB-Zentren als wichtiges Element, dass die Verbindung zwischen potentiellen universitären Gründern und dem „universitätsfernen“ Fördersystem herstellt. Anzumerken ist, dass die AplusB-Zentren thematisch deutlich breiter als aufgestellt sind als PreSeed/Seed und auch von einem breiteren Innovationsbegriff ausgehen als das im hochselektiven, bewusst auf Hochtechnologie abzielenden JITU-Programm der Fall ist. Daher macht eine projektbezogene Zusammenarbeit auch nicht in jedem Fall Sinn.

Governance von i2 - Business Angels

Das Programm ist sehr einfach und daher auch unbürokratisch aufgebaut. Potentielle Business Angels können sich einfach registrieren lassen, wobei jedoch von Seiten der aws darauf hingewiesen wird, dass eine Investmentaktivität in (riskante) Jungunternehmen erst ab einer bestimmten Vermögenshöhe tatsächlich Sinn macht.

Bei den nach Investoren suchenden Unternehmen wird von Seiten des 12 Teams (vier Personen) eine Bewertung durchgeführt und gegebenenfalls interne aws-ExpertInnen (z.B. Prüfung der Technologie, Marktchancen etc.) beigezogen. Dies führt zu einer Selektion, wobei diese in den vergangenen Jahren bewusst strenger wurde um die potentiellen Business Angels nicht mit einer Listen von kapitalsuchenden Unternehmen zu „überfluten“. Tatsächlich hat ja diese strengere Selektion bereits zu einer Steigerung der Vermittlungschancen geführt (wie in Kapitel 3.6 gezeigt wurde).

Diese so selektierten Projekte werden dann regelmäßig an die im Netzwerk registrierten Business Angels geliefert. Ein Ex-ante Versuch eines direkten, themenbezogenen Matchings (z.B. diese Gruppe von Business Angels erhält nur die Liste von IKT-relevanten Projekten) erfolgt bewusst nicht, da nicht alle Business Angels auf bestimmte Branchen / Technologiezweige fokussiert sind, sondern ihre Investments durchaus auch thematisch streuen. Unter Umständen kann dies (sollte sich die Startup Szene in den nächsten Jahren dynamisieren), dass diese Listen zu umfangreich würden. Die Antwort des 12 Teams auf dieses Problem läge dann in einer nochmaligen Erhöhung der Selektivität, wobei es trotzdem gilt die Balance zwischen zu hoher Selektion und zu breiter Auswahl zu finden. Sollte sich nämlich herausstellen, dass das 12 Team regelmäßig Projekte weg selektiert, die aber auf anderen Kanälen Business Angels finden, würde das die Anreize einer Beteiligung an 12 sowohl für kapitalsuchende Unternehmen als auch für Business Angels reduzieren.

$\mathrm{Zu}$ betonen ist, dass die bereits vor längerer Zeit erfolgt de facto Abschaffung der Vermittlungsprovision nunmehr seit ca. drei Monaten auch offiziell aus den Geschäftsbedingungen getilgt wurde.

Einschätzung der Eignung der derzeitigen Kennzahlen und Indikatoren zur Bewertung der Zielerreichung

Die JITU-Richtlinie legt folgenden Grundsatz bezüglich der Auswahl möglicher Indikatoren zur Bewertung der Zielerreichung fest „Die Ziele müssen in nachvollziehbarer Weise begründet sein, operationalisierbar und an Hand von qualitativen bzw. quantitativen Indikatoren überprüfbar sein. “ 
Im Folgenden wird die Liste der definierten Indikatoren wieder gegeben, wobei die fett dargestellten als Kernindikatoren vorgeschlagen werden, wobei die Auswahl vor allem aufgrund der Operationalisierbarkeit und der (laufenden) Verfügbarkeit der entsprechenden Daten erfolgte.

Indikatoren PreSeed

1. Steigerung der Gründungsbereitschaft im High Tech Bereich

2. Anzahl von High-Tech Vorgründungsprojekten (15 - 20 PreSeed Projekte jährlich bei zeitgerechter Dotation des Förderbudgets)

3. Anzahl und Anteil der aus PreSeed Projekten gegründeten Unternehmen (Ziel beim Anteil: 60 $80 \%)$

4. Größe des Projektteams (w/m)

5. Anzahl der PreSeed Anfragen

6. geplante/tatsächliche Projektkosten [EUR]

7. Gesamtprojektkosten/geförderte Projektkosten

8. geschaffene Arbeitsplätze $(\mathrm{w} / \mathrm{m})$ nach Sektoren, Technologiefeld und Bundesland

Indikatoren Seedfinancing

1. Forcierung und Etablierung Österreichs als Hochtechnologiestandort; gemessen an der Anzahl neu gegründeter und etablierter Unternehmen (Spin Offs, Spin Outs, akademische und nicht akademische Gründungen etc.)

2. Steigerung der Gründungsbereitschaft im High Tech Bereich;

3. Anzahl von High-Tech Unternehmen (10 - 15 Unternehmen jährlich bei zeitgerechter Dotation des Förderbudgets)

4. Anzahl der Seedfinancing Anfragen

5. Anzahl der geförderten Seedfinancing Unternehmen

6. Größe des Projektteams (w/m)

7. Fördervolumen [EUR]

8. geschaffene Arbeitsplätze $(w / m)$

9. Anzahl der erfolgreichen Rückführungen (High Flyers)

10. Insolvenzen

11. Rückflüsse

12. Höhe und Qualität von weiterer Finanzierung der Seedfinancing-Unternehmen; Weiter- bzw. Ausfinanzierung von High-Tech Unternehmen über mindestens weitere 12 Monate nach Auszahlungszeitraum

13. Mobilisierung von Venture Capital

14. geschaffene Arbeitsplätze $(\mathrm{w} / \mathrm{m})$ nach Sektoren, Technologiefeld und Bundesland 
Indikatoren MaZ

1. die Anzahl der eingereichten Projekte aufgeteilt nach Technologiefeldern und Bundesländern

2. die Anzahl der geförderten Unternehmen aufgeteilt nach Technologiefeldern und Bundesländern

3. Kosten des ExpertInneneinsatzes bezogen auf Sicherung des Investments der öffentlichen Hand und der Zielerreichung des Einsatzes

4. Steigerung der Überlebenswahrscheinlichkeit des Unternehmens nach Abschluss des Beratereinsatzes, Steigerung des Umsatzpotenzials bzw. des Finanzierungspotenzials bei entsprechenden Projekten

\subsection{Zielerreichung der Programmindikatoren}

Da für alle Module auch quantitative Zielindikatoren definiert sind, lässt sich die Zielerreichung anhand des verfügbaren Datenmaterials sehr einfach ermitteln. Tabelle $\mathbf{1 1}$ stellt den Zielwerten für die verschiedenen Zielindikatoren der drei JITU-Module sowie 12 den jeweils erreichten Istwerten gegenüber, wobei sowohl der Durchschnitt für die gesamte Evaluierungsperiode 2007 bis 2013 als auch die jährlichen Istwerte für die drei letzten Jahre (2011 bis 2013) angegeben sind.

Seedfinancing

Bei Seedfinancing liegen beim zentralen Zielindikator, nämlich die pro Jahr tatsächlich geförderten Unternehmen, im Durchschnitt der Jahre 2007 bis 2013 mit 13 geförderten Unternehmen genau im Zielband (10 bis 15 per annum). Bemerkenswert ist, dass in den letzten Jahren die Zahl der geförderten Unternehmen sehr stark angestiegen ist (von $15 \mathrm{im}$ Jahr 2011 auf 26 im Jahr 2013). Inwieweit dies auch Ausdruck einer, aufgrund von qualitativen Einschätzungen vieler Beobachter, wahrgenommenen Dynamisierung der technologie- und innovationsorientierten Startup-Szene Österreichs ist, bleibt jedoch noch abzuwarten. Die Zahl der Projektanträge liegt mit durchschnittlich 37 etwas unter dem Zielwert von 50, allerdings zeigt sich auch diesbezüglich ein Anstieg in den letzten drei Jahren und mit 46 Projektanträgen im Jahr 2013 lag dieser Wert annähernd am Zielwert. Mit ca. 24 \% ist die Insolvenzquote sehr niedrig, was einerseits für die Qualität der Selektion der Projekte durch die aws spricht und andererseits auch ein Anzeichen dafür ist, dass durch den Finanzierungsbeitrag der Förderung sowie die laufende Betreuung durch die aws der Unternehmensaufbau in den ersten Jahren gesichert wird und das Risiko des Scheiterns in der Frühphase reduziert wird.

PreSeed

Auch bei PreSeed werden die Zielindikatoren erfüllt, wobei sich auch hier am aktuellen Rand ein Trend zur „Übererfüllung“ abzeichnet. Über den gesamten Evaluierungszeitraum liegt die durchschnittliche Zahl der geförderten Gründung mit 20 p.a. bereits am oberen Rand des Zielbandes (15-20), während in den letzten beiden Jahren (2012 und 2013) dieser Zielwert bereits übertroffen wurde. Die „Erfolgsquote“ (tatsächlich erfolgte GmbH-Gründungen) liegt in den Jahren 2006 bis 2011 immer im Zielband von 60 bis 80 \%. Hierbei ist anzumerken, dass für die Jahre 2012 und 2013 dieser Zielindikator noch nicht abschließend berechnet werden kann, da die Zeitdauer zwischen Förderungsperiode und formaler Gründung sich teilweise über Jahre hinstreckt (vgl. Kapitel 3.2). 
Tabelle 11: Zielerreichung Programmindikatoren

\begin{tabular}{|c|c|c|c|c|c|}
\hline & Zielwert & 2011 & 2012 & 2013 & $\varnothing$ 2007-2013 \\
\hline \multicolumn{6}{|l|}{ Seedfinancing } \\
\hline Anfragen p.a.* & 50 & 38 & 40 & 46 & 37 \\
\hline geförderte Unternehmen p.a. & $10-15$ & 15 & 18 & 26 & 13 \\
\hline Insolvenzen & $<50 \%$ & $23,4 \%$ & $24,3 \%$ & $24,2 \%$ & - \\
\hline \multicolumn{6}{|l|}{ PreSeed } \\
\hline Anfragen p.a.* & & 41 & 81 & 90 & 59 \\
\hline geförderte Unternehmen p.a. & $15-20$ & 18 & 27 & 23 & 20 \\
\hline Gründungen nach Abschluss & $60-80 \%$ & \multicolumn{4}{|c|}{ 2006-2011: zwischen 60 und 80\% } \\
\hline \multicolumn{6}{|l|}{ MaZ } \\
\hline geförderte Unternehmen & $15-20$ & 1 & 3 & 1 & $21^{* *}$ \\
\hline \multicolumn{6}{|l|}{ ¡2 Business Angels } \\
\hline Investoren unter Vertrag p.a. & 120 & 140 & 179 & 205 & 145 \\
\hline Unternehmensanfragen p.a. & $350-400$ & 416 & 479 & 457 & 392 \\
\hline Unternehmenslistungen p.a. & ca. 50 & 58 & 61 & 60 & 51 \\
\hline Vermittlungen p.a. & 5 & 8 & 10 & 18 & 8 \\
\hline Einnahmen p.a. & 30.000 & 35.220 & 39.900 & 41.850 & 32.936 \\
\hline
\end{tabular}

* Daten beziehen sich auf Programmlinie IKT\&PHS.

$* * \Sigma 2007-2013$

Quelle: aws, eigene Berechnungen.

Management auf Zeit

Der für MaZ anvisierte Zielwert von 15 bis 20 geförderten Unternehmen konnte im Evaluierungszeitraum mit 21 Förderungen ebenfalls erfüllt werden, wobei sich zu Beginn der Programmlaufzeit viele Förderfälle aus dem bestehenden Seedfinancing-Portfolio ergaben. In den Folgejahren ist die Zahl der Förderungen dann zurückgegangen.

I2 - Business Angels

Die Zielindikatoren beim Programm 12 werden für den gesamten Evaluierungszeitraum teilweise übererfüllt (mit durchschnittlich acht erfolgreichen Vermittlungen pro Jahr und 145 Investoren unter Vertrag liegen diese Indikatoren merklich über den Zielwerten). Für die letzten drei Jahre zeigt sich sogar eine deutliche Übererfüllung, die Istwerte übertreffen die Zielwerte beträchtlich. Dies trifft vor allem auf die Zahl der Business Angels als auch auf die Zahl der erfolgreichen Vermittlungen zu (wobei hier insbesondere das Jahr 2013 mit 18 erfolgten Vermittlungen herausragend ist).

\subsection{Ergebnisse der Online Befragung und internen Evaluierung}

Um das Programm aus der Perspektive der geförderten Unternehmen darstellen zu können wird im Folgenden auf eine Online-Befragung bei PreSeed und Seedfinancing geförderten Unternehmen aus dem Sommer 2013 zurückgegriffen ${ }^{19}$. Diese Online-Erhebung umfasste folgende drei Fragenblöcke:

- $\quad$ Fragen zu den Motiven für die Unternehmensgründung, womit aufgezeigt werden soll, inwieweit aus den Motiven der Unternehmensgründer der hohe, selbst gesetzte Anspruch des JITUProgramm an den Innovationsgehalt bestätigt werden kann.

${ }^{19}$ Gassler et al., 2013 
- Fragen zu Barrieren bzw. Hemmnissen im Gründungsprozess, womit analysiert werden soll, inwieweit JITU mit seinen spezifischen Förderansatz die tatsächlich auftretenden Hemmnisse der Gründer adressiert.

- $\quad$ Fragen zu Bedeutung der aws-Förderung (PreSeed und Seed) für die Unternehmensgründung bzw. den Unternehmensaufbau sowie zur Einschätzung der Qualität der Betreuung durch die aws. Hiermit können Aussagen zur Additionalität der Förderung getroffen werden und die subjektive Einschätzung der Betreuungsqualität und Förderungsabwicklung durch die Fördernehmer aufgezeigt werden kann.

Bei den Ergebnissen ist zu beachten, dass die Befragen eine Einschätzung auf einer Skala von 0 bis 100 vornahmen, wobei diese Einschätzung mittels eines frei verschiebbaren Reglers (d.h. die Befragten mussten keine exakte Zahl angeben) vorgenommen wurde.

In Abbildung $\mathbf{5 0}$ sind die Ergebnisse zum Fragenblock „Motive zur Gründung“ dargestellt und zwar wiederum in Form von Boxplot Charts.

Die Umsetzung einer neuen, innovativen Geschäftsidee und das Erkennen einer Marktlücke mit Wachstumspotential sind eindeutig und mit hohem Abstand die beiden wichtigsten Gründungsmotive ${ }^{20}$. Bei beiden Motiven liegen der Mittelwert (0:= „keine Relevanz"; 100:= „sehr hohe Relevanz") um die 90 und der Median sogar bei 100 bzw. knapp darunter. Dass beide Motive eine derart hohe Relevanz aufweisen, ist ein Hinweis darauf, dass im JITU-Programm tatsächlich auf einen hohen Innovationsgrad fokussiert wird und die geförderten Gründungen diesen Anspruch erfüllen. Offensichtlich schätzen die Gründer ihr Wachstumspotential sehr hoch ein und sind auch bereit zu Wachstum. Dies ist wirtschaftspolitisch deshalb von großer Bedeutung, da viele Gründer ihr Unternehmen oft ohne grundsätzliche Bereitschaft zu einem merklichen Wachstum gründen und sich bewusst für dauerhafte „Kleinheit“ entscheiden. Diese prinzipielle Skalierbarkeit und die Wachstumsbereitschaft sind auch wichtig, da dies ein notwendiges Kriterium für die „Risikokapitalfähigkeit“ von Unternehmen in der Frühphase darstellt.

\footnotetext{
${ }^{20}$ Das Restmotiv "Sonstige" wird hier nicht berücksichtigt, da es diesbezüglich sehr wenige Antworten gab.
} 


\section{Abbildung 50: Gründungsmotive (0 wenig Relevanz, 100 sehr hohe Relevanz)}

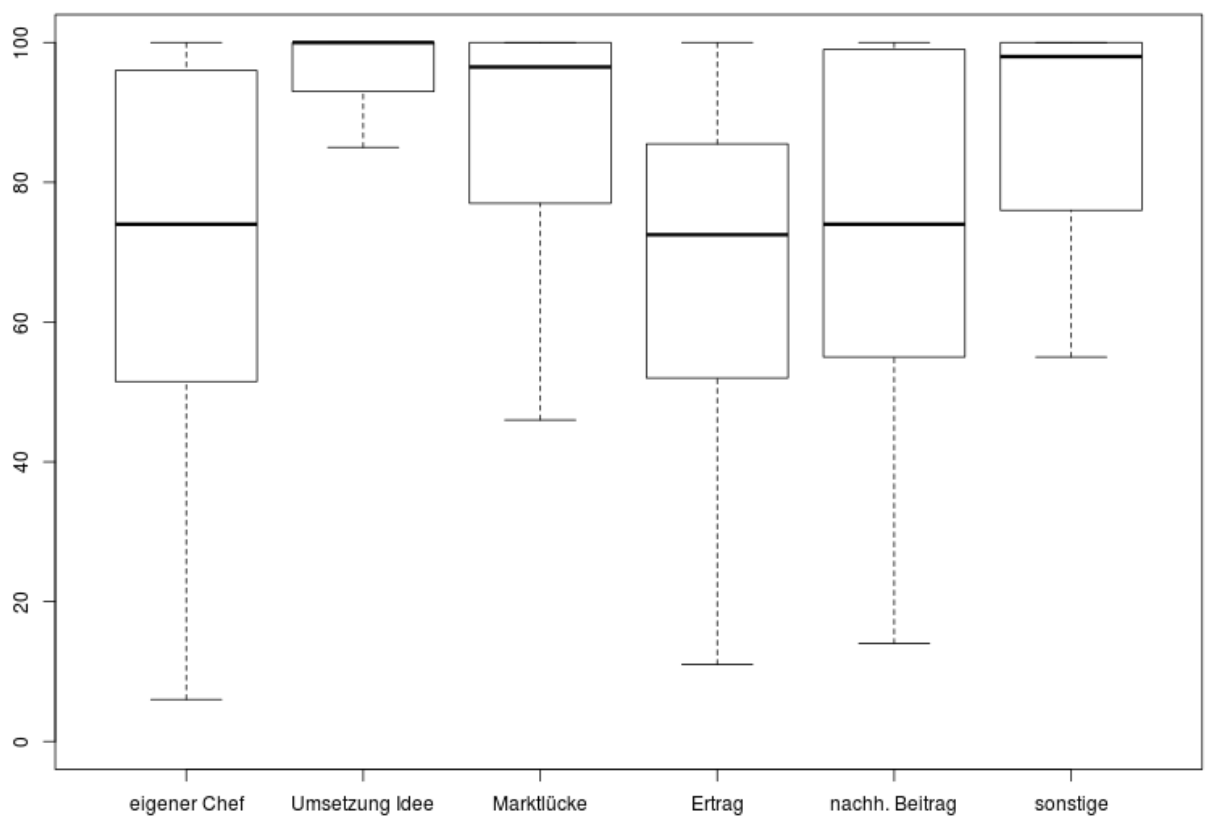

Quelle: Onlinebefragung 2013. Anmerkung: $n=49$.

Abbildung $\mathbf{5 1}$ gibt die Ergebnisse des zweiten Fragebogenblocks der Online-Befragung wieder (Gründungshemmnisse). Dabei zeigt sich, dass das Finanzierungshemmnis als am bedeutsamsten eingestuft wird (Median ca. 80, Mittelwert ca. 65). Allerdings ist die Spannweite (die Antennen reichen von 0 bis 100) doch sehr weit und immerhin $25 \%$ der Befragten gaben einen Wert von ca. unter 30 an. Die Ergebnisse können einerseits dahin interpretiert werden, dass mit dem JITU-Programm Seedfinancing als Finanzierungshilfe ein der zentralen Gründungshemmnis adäquates Instrument zur Anwendung kommt und anderseits, dass es eben gerade die Finanzierungshilfe durch Seedfinancing ist, die dazu führen, dass doch auch einige Gründer die finanziellen Hürden als weniger bedeutsam einstufen.

\section{Abbildung 51: Gründungshemmnisse}

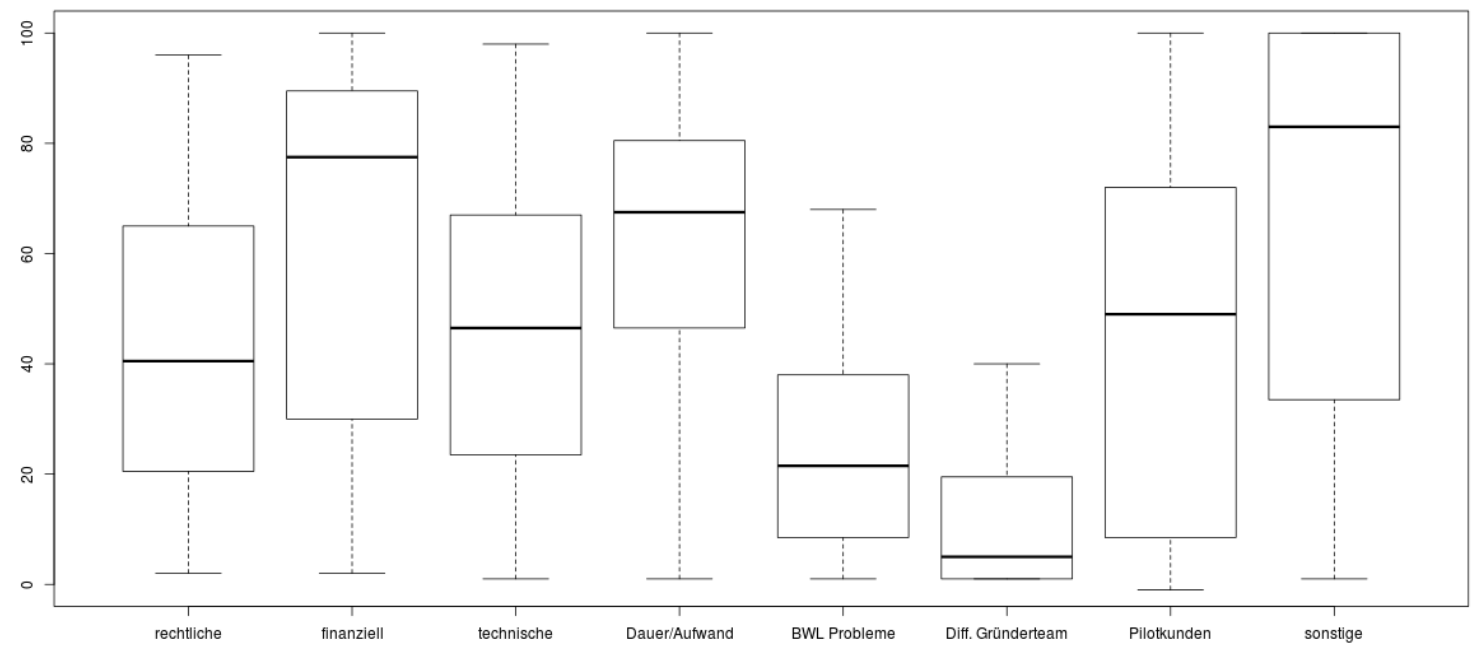

Quelle: Onlinebefragung 2013. Anmerkung: $n=49$.

Lange Zeitdauer bzw. hoher organisatorischer Aufwand folgt an zweiter Stelle der wahrgenommenen Gründungshemmnisse. Nachdem das JITU-Programmmodul PreSeed u.a. auf die Erleichterung des 
Gründungsprozesses per se abzielt, wird auch diese zweite, zentrale Gründungshemmnis vom JITUProgramm explizit adressiert (vgl. dazu auch die Diskussion der Ergebnisse der aws-internen Evaluierung weiter unten).

Der dritte Fragenblock beziehe sich zum einen auf die Einschätzung der Bedeutung der aws-Förderung für die Unternehmensgründung bzw. die darauf folgende Frühphasenentwicklung und zum anderen auf eine Bewertung verschiedener Aspekte des Programms bzw. der Betreuungsqualität von Seiten der aws.

Abbildung 52 zeigt, dass insgesamt wird der aws-Förderung eine sehr hohe Bedeutung für den Gründungsprozess zugeschrieben wird. Der Mittelwert liegt bei annähernd 95 Punkten, der Median noch etwas darüber. Die Box ist sehr klein und die untere Antenne reicht nur bis etwas oberhalb von 80 Punkten, was bedeutet, dass diese Einschätzung einer hohen Relevanz der aws-Förderung durchgängig von allen Befragten geteilt wird. Letztlich ist dieses Ergebnis auch ein Hinweis darauf, dass durch das JITU-Programm eine hohe Additionalität bewirkt wird, da anzunehmen ist, dass ohne der substantiellen Förderung viele der Gründungsprojekte nicht erfolgreich durchgeführt hätten werden können.

Abbildung 52: Bedeutung PreSeed/Seed für den Gründungsprozess bzw. den Unternehmensaufbau aus Sicht der Fördernehmer

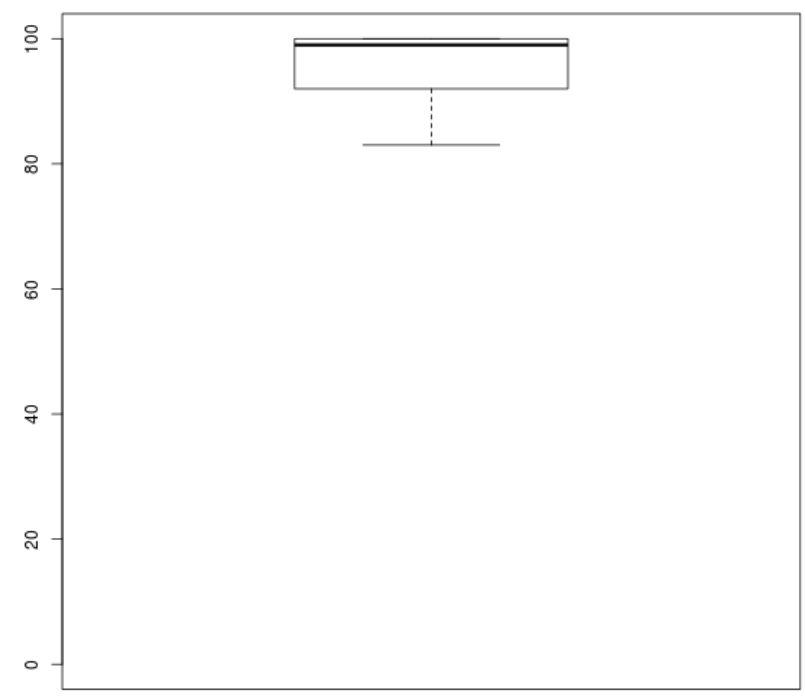

Quelle: Onlinebefragung 2013, $n=49$.

Abbildung 53 gibt die Ergebnisse der Befragung bezüglich der Programmeinschätzung (einschließlich der "soft measures" des Programms) sowie die Bewertung der Förderabwicklung (z.B. notwendiger bürokratischer Aufwand) und Betreuungsqualität wider. Die Antworten sind für die meisten der abgefragten Aspekte positiv bis sehr positiv. Besonders positiv werden der persönliche Kontakt zum zuständigen aws-Referenten (was auch ein Hinweis auf hohe Qualität der Förderabwicklung ist), die Eignung der Förderung bei Vorhaben mit technischen Risiken sowie die Professionalität der Abwicklung hervorgehoben. Die Beratung in Bezug auf die Förderungsmodalität kann wird ebenfalls sehr positiv bewertet. Die schlechteste Bewertung erfolgt hinsichtlich der Beratung in punkto Suche / Finden von privaten Risikokapitalgebern, wobei hier die Spannweite aber sehr hoch ist (Antennen von 0 bis 100). Einerseits konnten viele der geförderten Gründungen noch keine zusätzlichen Risikokapitalgeber finden und mögen deswegen die einschlägige Beratung durch die aws negativ bewerten und andererseits bewerten jene Gründer, die bereits erfolgreich Risikokapitalgeber im Unternehmen aufweisen, wahrscheinlich dann auch die aws-Beratung diesbezüglich eher positiv. 
Abbildung 53: Programmeinschätzung aus Sicht der Fördernehmer
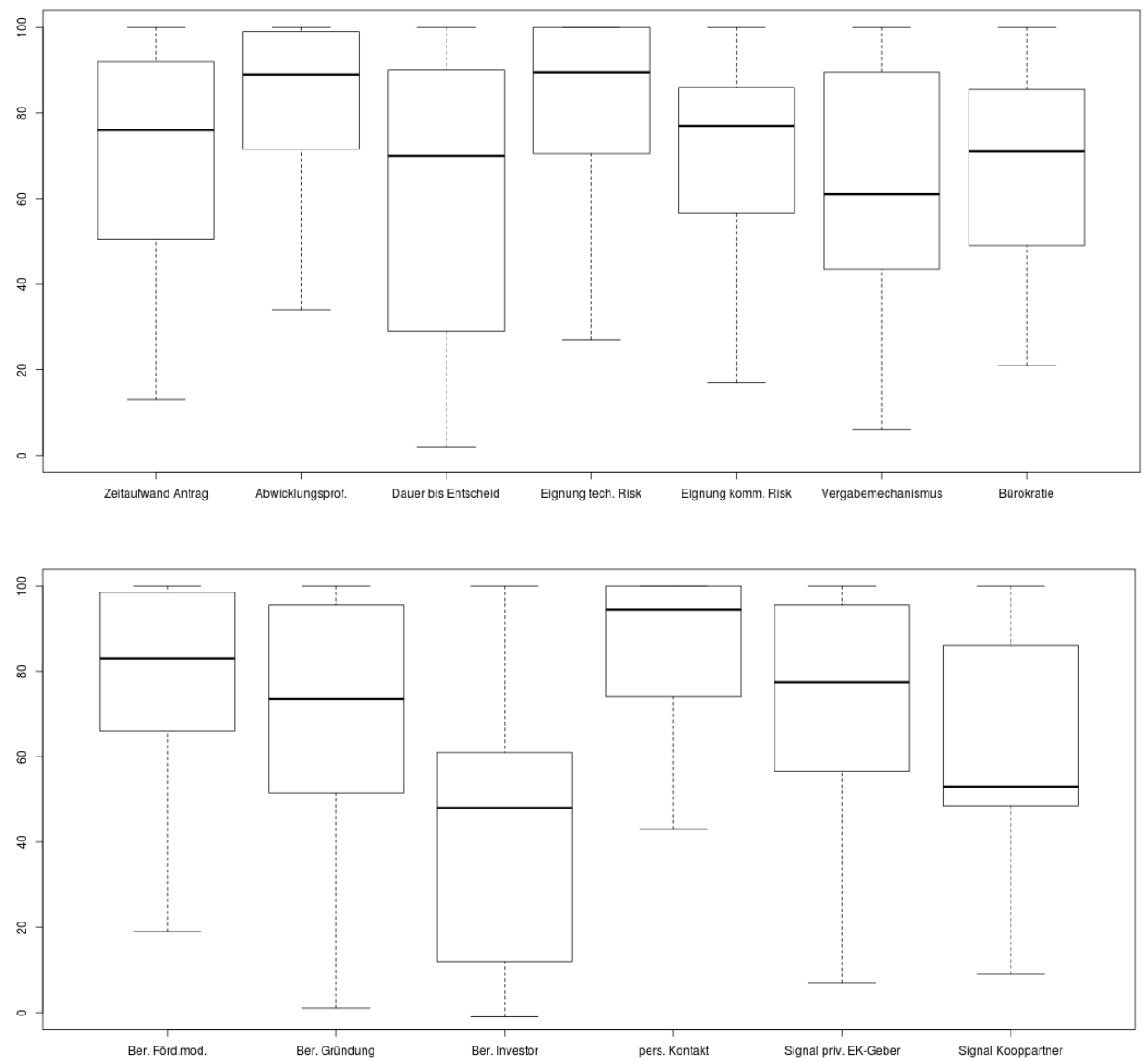

Quelle: Onlinebefragung 2013, $n=49$.

Im Folgenden werden die Ergebnisse der aws-internen Evaluierung, die auf einer Online-Befragung (durchgeführt im Dezember 2012) beruhen, diskutiert, wobei aussagefähige Antworten von 23 (bzw. im Fall einer Fragengruppe 25) durch Seedfinancing geförderten Unternehmen vorliegen. Zu beachten ist, dass sich die Werte in den folgenden Abbildungen Abbildung $\mathbf{5 4}$ bis Abbildung $\mathbf{5 9}$ auf die jeweiligen Prozentanteile beziehen, also keine Bewertungsskala darstellen. Die Ergebnisse dieser Befragung lassen sich wie folgt zusammenfassen:

- $\quad$ Die Förderung durch Seedfinancing führt zu einer deutlichen Verbesserung der Finanzierungsbasis des Unternehmens. Mehr als $90 \%$ der befragten gaben an, dass die Unterstützung durch das Förderprogramm ihre Finanzierungstruktur sowie ihre finanziellen Entscheidungsgrundlagen verbessert hat. Somit ist eines der wesentlichen Programmelemente, nämlich einen Beitrag zur Finanzierung des Unternehmensaufbaus zu leisten, erfüllt (vgl. Abbildung 54)

- Mehr als $90 \%$ der Befragten gaben an, dass die Förderung einen deutlichen Einfluss auf die Zeitdauer der Projektumsetzung zur Folge hatte (und $87 \%$ gaben an, dass Seedfinancing eine positive Wirkung auf die Realisierbarkeit ihres Vorhabens zeitigte) (vgl. Abbildung 55). Nachdem bei den Gründungshemmnissen die Zeitdauer der Unternehmensgründung bzw. des Unternehmensaufbaus als ein wesentliches Hemmnis (nach den Finanzierungsrestriktionen) genannt wurde, zeigt dies, dass das Programm auch diesbezüglich auf die Bedürfnisse von neu gegründeten bzw. jungen Unternehmen hin zugeschnitten ist. Der hohe Wert in Bezug auf die 
Erhöhung der Realisierungschancen deutet wiederum auf einen hohen Additionalitätseffekt hin, ohne Förderung wären u.U. viele der Projekte bereits in der Frühphase gescheitert.

- Nachdem Seedfinancing als ein hoch selektives, auf technologische Innovationen abzielendes Programm konzipiert ist, stellt sich die Frage, inwieweit die Innovationskompetenz der geförderten Unternehmen beeinflusst werden konnte. Abbildung 56 zeigt, dass 87 \% der Befragten angaben, dass Seedfinancing dazu beitrug, dass ein erstmaliger Marktauftritt mit innovativen Produkten möglich war. Nachdem es eine explizites Ziel der aktuellen FTI-politischen Strategie der österreichischen Bundesregierung ist, den Anteil von genuinen Innovationen („neu für den Markt“) am gesamten Innovationsgeschehen in Österreich zu erhöhen, leistet also das Seedfinancing Programm hierfür ebenfalls einen Beitrag.

- $\quad$ Ein explizites Ziel der öffentlichen Förderung mittels Seedfinancing ist es, dass die geförderten Unternehmen nach erfolgreichem Durchlaufen der frühesten Unternehmensentwicklungsphase auch am privaten Risikokapitalmarkt eine entsprechende Finanzierung finden („Hebeleffekt“ der Förderung). Wie aus Abbildung 57 ersichtlich ist, geben $84 \%$ der Befragten an, dass ihnen die Förderung die Chance auf Akquise von Finanzierung durch private Eigenkapitalgeber erhöht hat.

- Seit vielen Jahren ist die Erhöhung der F\&E-Quote Österreichs ein zentrales Ziel der österreichischen Forschungs- und Technologiepolitik. Aus Abbildung $\mathbf{5 8}$ folgt, dass durch die Förderung die F\&E-Aktivitäten (sowohl monetär als auch personell) ausgeweitet werden konnten. Darüber hinaus unterstreicht der hohe Wert (92\%) bei der Frage nach Erreichung einer Technologieführerschaft im Marktsegment den hohen Innovationsgrad.

Abbildung 54: Effekt der Förderung auf Finanzierungsbasis im Seedfinancing

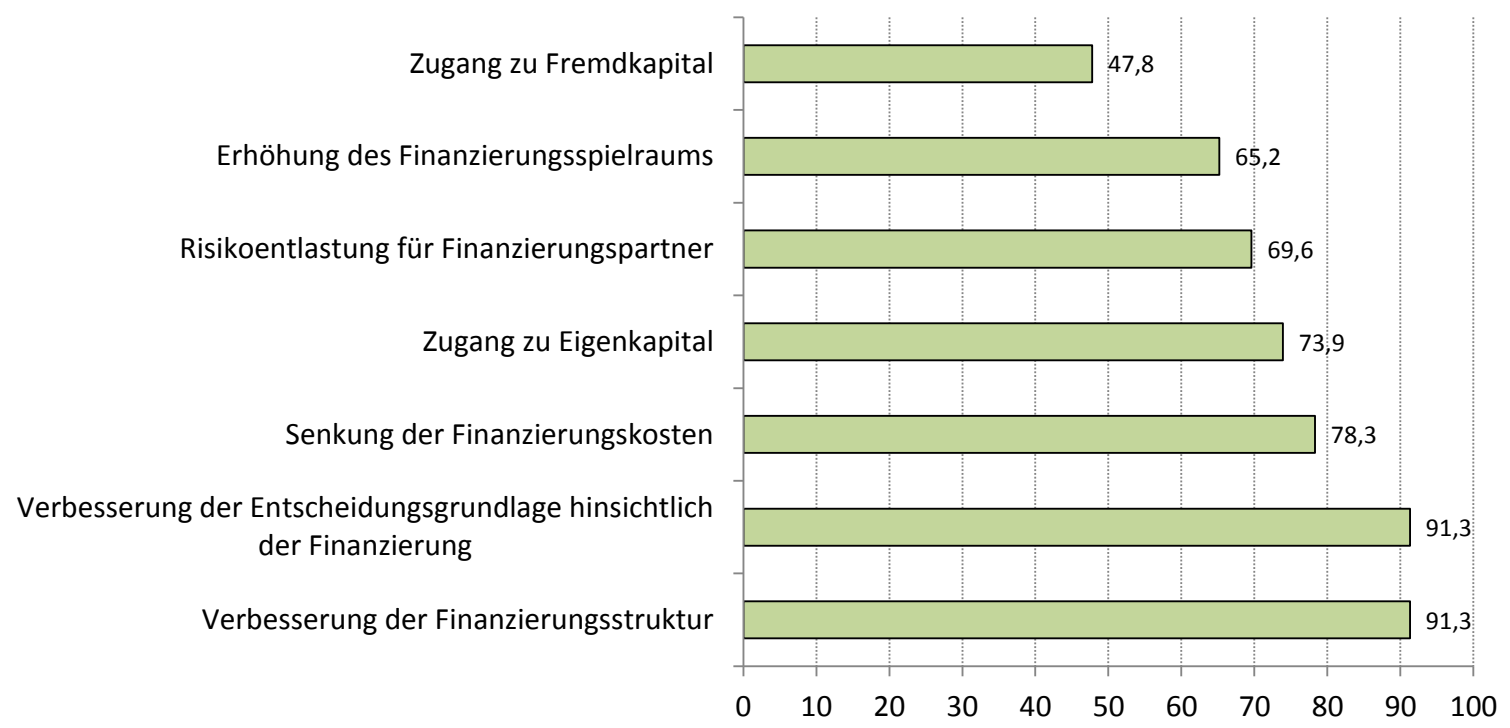

Quelle: aws - Interne Evaluierung Seedfinancing 2012. Anmerkung: $n=23$. 


\section{Abbildung 55: Effekt der Förderung auf Projektumsetzung im Seedfinancing}

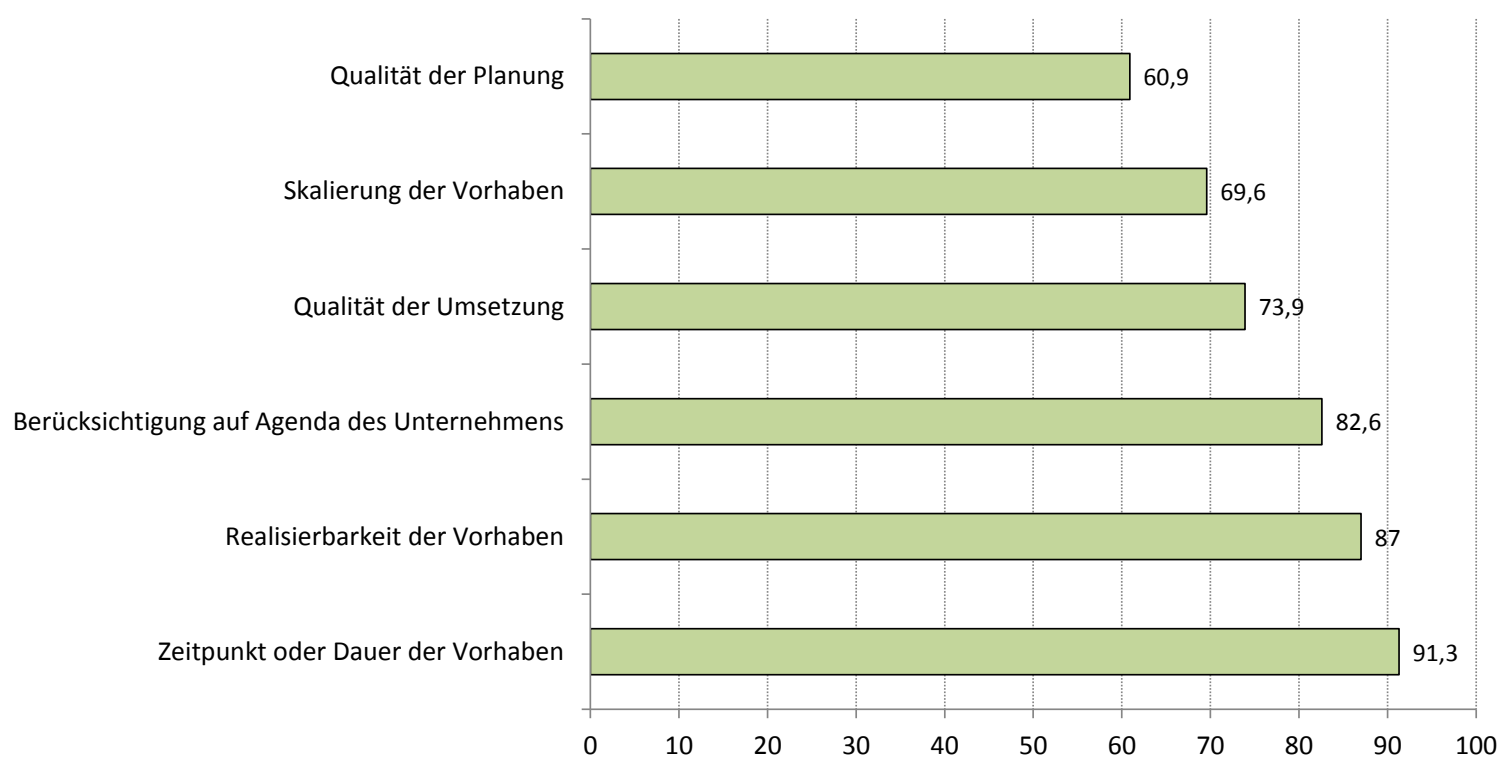

Quelle: aws - Interne Evaluierung Seedfinancing 2012. Anmerkung: $n=23$.

\section{Abbildung 56: Projektwirkung im Seedfinancing auf Innovationsaktivitäten und -kompetenzen}

Ausweitung des Umsatzes mit Innovationen

Verbesserung des Wissens über Finanzierungserfordernisse und -optionen

Verbesserung der Kompetenzen zum Management von Innovationsprozessen

Erlernen neuer Kompetenzen für den Markterfolg von Innovationen

Erstmaliger Marktauftritt mit innovativen Produkten

Erfahrungsgewinn zu Management und Bewertung von Kooperationen

Erfahrungsgewinn zu Einschätzung und Festlegung von Innovationszielen

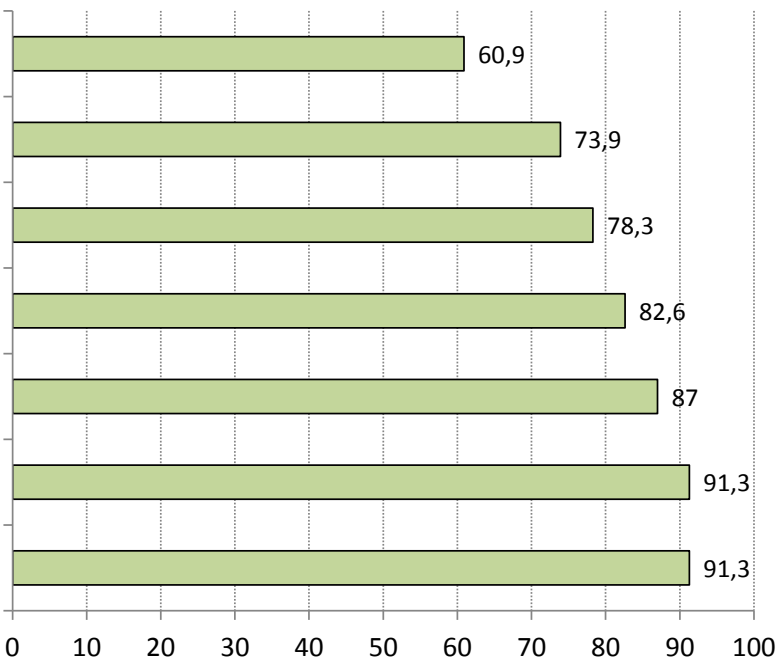

Quelle: aws - Interne Evaluierung Seedfinancing 2012. Anmerkung: $n=23$. 


\section{Abbildung 57: Teilnahme an aws-Förderung bewirkt ... - eher und sehr vorteilhaft}

verbessert Bonität ggü. Bank

verbessert die Verhandlungsposition ggü. Bank

reduziert Übertragung von Sicherheiten an Bank

geringere Zinsbelastung

senkt die Finanzierungskosten des Vorhabens

Verbesserung Entscheidungsgrudlagen Finanzierungsoption

ermöglicht Projekte die für andere Kapitalgeber zu riskant sind

erhöht die Chancen auf priv. EK-Geber

Verbesserung Entscheidungsgrudlagen des Unternehmens oder dessen Partner

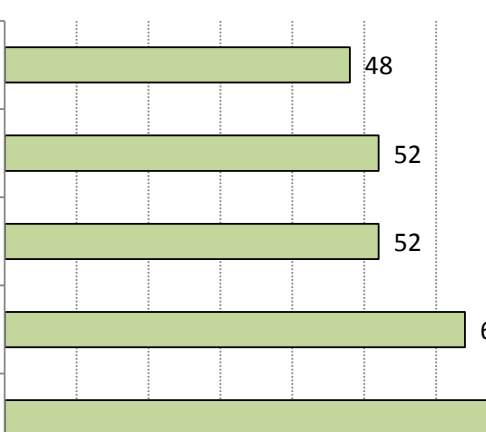

64
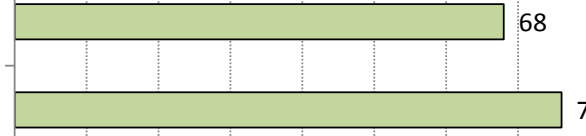

76
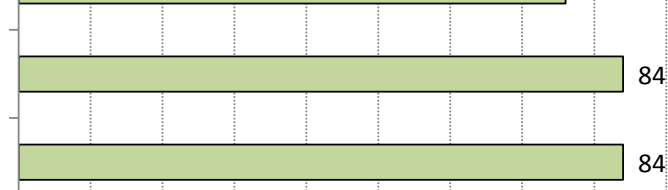

84

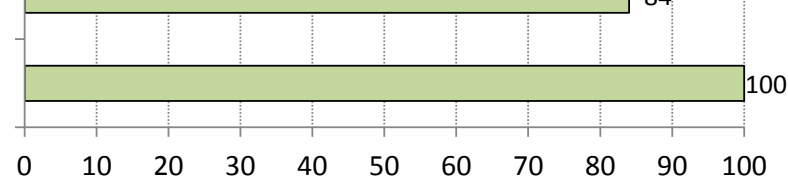

Quelle: aws - Interne Evaluierung Seedfinancing 2012. Anmerkung: $n=25$.

Abbildung 58: Aus der Projektumsetzung folgt ... - Antwort: Ja

verbessert Chancen von Frauen ggü. Männern

positive Effekte auf die Beschäftigung von Frauen

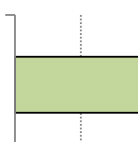

nachhaltige Absicherung von Beschäftigung

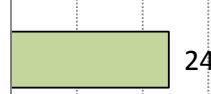

24

erhöht die F\&E-Personalquote

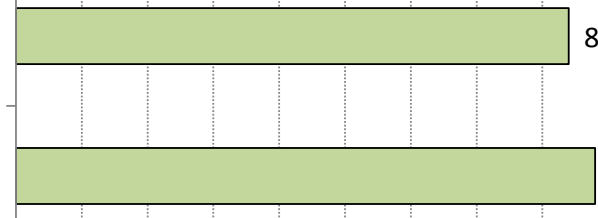

84

88

merkbare Ausweitung der Beschäftigung

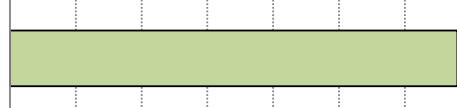

68

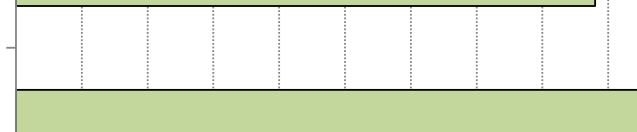

ist mit einem Zuwachs an F\&E verbunden

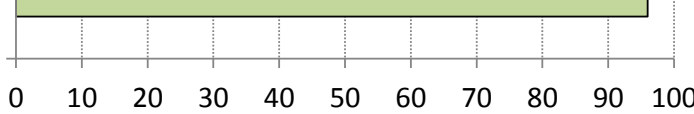

Quelle: aws - Interne Evaluierung Seedfinancing 2012. Anmerkung: $n=25$. 


\section{Abbildung 59: Das Vorhaben leistet einen Beitrag für ... - Antwort: Großer Beitrag}

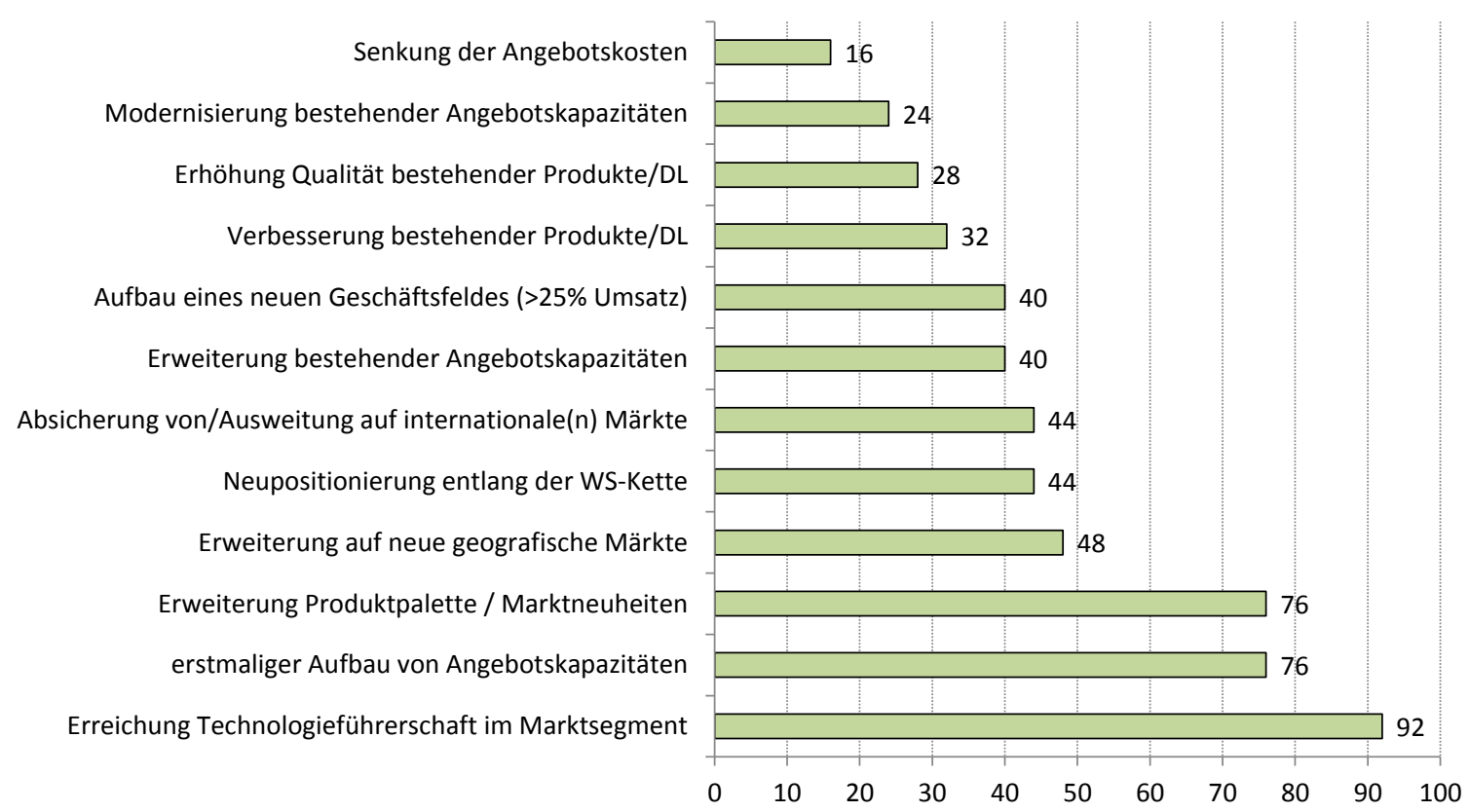

Quelle: aws - Interne Evaluierung Seedfinancing 2012. Anmerkung: $n=25$.

\subsection{Synthese und Empfehlungen}

In Abbildung 60 wird auf Basis des vorhandenen Datenmaterials gezeigt, dass mit höherer Fördersumme (jeweils pro Jahr) auch die Annahmequote steigt, wobei dies vor allem für Seedfinancing gilt. Bei LISA gilt dieser Zusammenhang zwar ebenfalls aber diesbezüglich konnte nicht zwischen PreSeed und Seedfinancing getrennt werden. Nach Aussagen der aws-Programmreferenten werden dabei aber keine Abstriche bezüglich der Projektkriterien getätigt, die strengen Auswahlkriterien gelten über alle Jahre hinweg gleich.

\section{Abbildung 60: Förderzusagen (in Mio. EUR) und Annahmequote, 2007-2013}

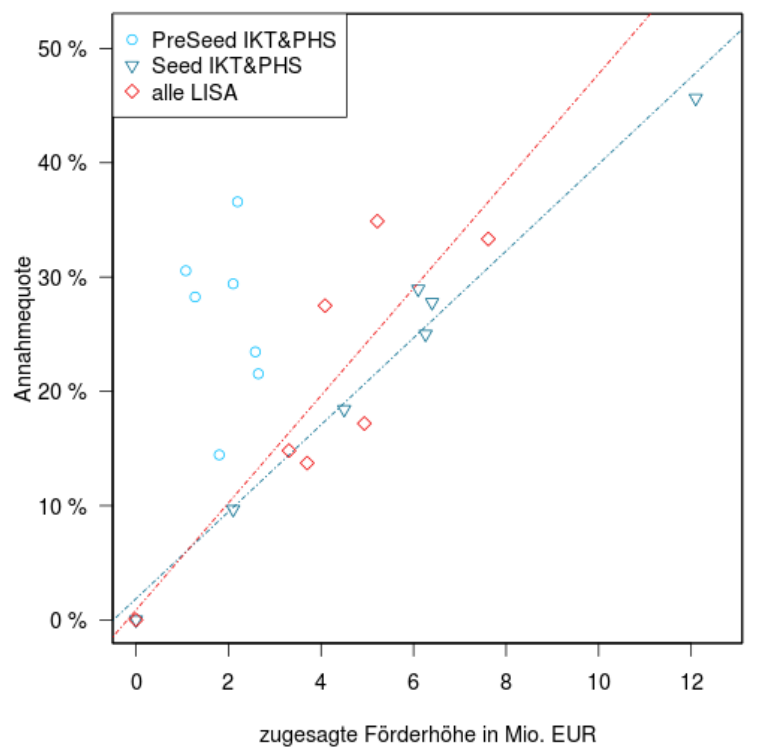

Quelle: aws, eigene Darstellung.

Die Tatsache, dass bei einer höheren verfügbaren Förderungssumme, aus der Gesamtzahl der Förderansuchen ein größerer Anteil genehmigt werden konnte, deutet darauf hin, dass das Potential 
qualitativ hochwertiger und chancenreicher High-Tech Gründungen in Österreich größer ist als mit der derzeit für das JITU-Programm verfügbaren Summe auch gefördert werden kann. Letztlich deutet dies darauf hin, dass bei einer Ausweitung der Fördersumme auch die Zahl der durchgeführten High-Tech Projekte steigen würde. Die Analyse der Programmentwicklungen liefert ebenfalls Anzeichen hierfür. So stieg die durchschnittliche Anzahl an jährlich zugesagten Förderungen der Programmmodule PreSeed und Seedfinancing von 30 (2008-2010) auf 42 (2011-2013) an.

Eine Steigerung der Projekte für sich betrachtet deutet jedoch noch nicht auf eine qualitativ-hochwertige Selektion hin. Die JITU-Richtlinien setzen diesbezüglich die Steigerung der Überlebensrate als Zielsetzung fest. Ein Vergleich der Insolvenzquoten der Seedfinancing-geförderten Unternehmen mit der Grundgesamtheit der österreichischen Unternehmensgründungen im allgemeinen und ähnlich riskanten Gründungsvorhangen (VC-Projekte) im speziellen, deutet auf eine geringere Ausfallswahrscheinlichkeit der Projekte hin. Zudem liegen die Beschäftigungswachstumsraten tendenziell über jenen der Vergleichsgruppe junger Unternehmen in Österreich, was verdeutlicht, dass die im Rahmen der JITU-Richtlinie geförderten Unternehmen durch die starke Selektion eine (im positiven Sinn) spezifische Subpopulation des gesamten Unternehmensbestands bzw. der Unternehmensneugründungen darstellen.

Mit steigendem Unternehmensalter der Begünstigten nimmt die Wahrscheinlichkeit privates Risikokapital von institutionellen VC-Fonds und von Business Angel zu akquirieren zu, was den Zielsetzungen der Hebelwirkung und Ausweitung der Kapitalbasis entspricht. In den Interviews mit den Programmverantwortlichen wurde hierzu auch bemerkt, dass bis dato noch keine PreSeed und Seedfinancing Projekte Anschlussförderungen in Form der Risikokapitalprogramme Business Angel Fonds und dem aws Gründerfonds erhalten haben. Dies lässt sich zumal darauf zurückzuführen, dass diese Programme erst seit kurzem (2013/2014) bestehen und die kleinen Fallzahlen bisher noch zu keiner Vermittlung geführt haben. Abseits der inhaltlichen Kriterien besteht bei diesen Förderprogrammen die Besonderheit, dass sich die privaten (BA) bzw. die institutionellen (Gründerfonds) Investoren bezüglich der Terms einig werden müssen. Dabei können eine Vielzahl an exogenen (andere externe Investoren involviert) und individuellen Faktoren (Gründer wollen nicht) auftreten, welche einen erfolgreichen Matching-Prozess im Wege stehen. Generell hat sich bei den Interviews mit Business Angels (wie auch mit Managern von institutionellen VC-Fonds) gezeigt, dass die Bewertung des Unternehmenswertes ein langwieriger Prozess ist, der sich üblicherweise über mehrere Monate hinweg zieht.

Die begleitende Förderung eines Unternehmens durch über die frühen und riskanten Lebensphasen mittels verschiedener Förderungen (Kombination und Vernetzung der Förderinstrumente) stellt einen dezidierten Wunsch der Förderinstitutionen dar, findet sich in dieser expliziten Form jedoch nicht in den Programmdokumenten. Aus gesamtökonomischer Sicht ist es relevant, dass das Marktversagen in der Anschlussfinanzierung von Hochtechnologie-Unternehmen behoben wird. Da die österreichische VCFrühphasenfinanzierung nach Meinung von Branchenkennern (Experteninterview mit AVCO, VC-FondsManagern, Business Angels) seit längerem auf kaum bemerkbarem Niveau stagniert, können die öffentlichen Ambitionen in diesem Segment als positiv im Sinne des Startup Ökosystems angesehen werden. Mit der Venture Capital Initiative (VCI), dem Gründerfonds, dem Business Angel Fonds und i2 Business Angels bestehen innerhalb der aws vier Programme welche durch ihre marktgerechte Ausgestaltung langfristige Stimuli für den österreichischen Risikokapitalmarkt entfalten können und somit diese Komponente des Startup Ökosystems abdecken.

Die in den letzten Jahren beobachtete Tendenz im steigenden Engagement von wohlhabenden Privatpersonen mit unternehmerischem Hintergrund ist hier besonders vielversprechend. Das JITUProgramm ergänzende i2 - Business Angels Programm liefert hier durch die unbürokratische Ausgestaltung und des Charakters der Matching-Plattform eine marktkonforme Möglichkeit zur Unterstützung dieser Entwicklungen. Stellt sich bei einigen der vermittelten Business Angels mit den Jahren ein positiver Track 
Record ein, so qualifizieren sich diese Investoren für eine Registrierung im Business Angel Fonds und können ihre Investments mit diesen Mitteln auf Basis einer Ko-Finanzierung hebeln.

Bevor die Empfehlungen hinsichtlich der JITU-Programmmodule und i2 - Business Angels abgegeben werden, soll noch kurz auf das allgemeine wirtschaftspolitische Umfeld für Start-Ups (riskante, wachstumsorientierte Unternehmensgründungen) in Österreich eingegangen werden. Im Rahmen der durchgeführten Interviews mit Start-Up Unternehmen im Hochtechnologiebereich wurde die hohe steuerliche Belastung und der hohe administrative Aufwand im Gründungsprozess bzw. danach thematisiert. Die Seedphase eines Hochtechnologieunternehmens zeichnet sich durch einen massiven Arbeitsaufwand von Seiten der Gründer bei oft geringen Erfolgsaussichten aus. Neben dem Druck schnell die Geschäftsidee marktfähig zu machen, Umsätze zu erzielen besteht die Herausforderung zusätzliches Personal zu bezahlen und administrieren. So wurde bspw. in einem Interview der „Manager auf Zeit" als zusätzliche Arbeitskraft angesehen, welche zur Bewältigung des administrativen Unternehmensleitungsaufwands eingesetzt wurde, um für die Dauer der Förderung eine Entlastung für die Gründer zu sein. Die Förderung deckt dabei Kosten für Steuern und Abgaben welche an anderen Stellen die Liquidität des Unternehmens belasten. Da die Anzahl an Hochtechnologiegründungen in Österreich überschaubar ist, würde eine allgemeine wirtschaftspolitische Empfehlung lauten, Start-Ups welche über öffentliche Stellen als innovativ, technologieintensiv und potentiell wachstumsstark eingestuft werden, zeitlich befristet steuerlich zu begünstigen (bzw. freizustellen). Aufgrund der bereits erfolgten Selektion auf Seiten der Förderstellen, würden keine zusätzlichen Transaktionskosten entstehen und aufgrund der geringen Anzahl und der zeitlichen Befristung würden die steuerlichen Ausfälle gering ausfallen.

\section{Empfehlungen und Schlussfolgerungen}

Auf Basis der quantitativen und qualitativen Analysen kommt das Evaluierungsteam zu folgenden Empfehlungen bzw. Schlussfolgerungen.

- Prinzipiell ist festzuhalten, dass das JITU-Programm von seiner Konzeption und seinem Instrumentarium her in geeigneter Art und Weise die in den Programmrichtlinien festgelegten Ziele (Steigerung von technologieorientierten Gründungen, Steigerung der Überlebensquote, Ermöglichen einer Anschlussfinanzierung von Risikokapital) zu adressieren. Die quantitativen Zielindikatoren werden in allen drei JITU Modulen de facto durchgängig erreicht bzw. tlw. übertroffen.

- Das JITU-Programm ist daher ein zentrales Element des österreichischen Start-Up Ökosystems und verfügt über zahlreiche komplementäre Schnittstellen $\mathrm{zu}$ anderen Gründungs- und Technologieförderungen. Eine weitere Optimierung der Zusammenarbeit (vor allem mit den AplusB Zentren) ist aber dennoch möglich (bspw. verstärkter Datenaustausch, gemeinsame Vorprüfung von Projekten) und wurde auch von beiden Seiten hervorgehoben.

- Die Analyse der Programmdaten lässt darauf schließen, dass mit den gegebenen Fördervolumen nicht alle den Kriterien entsprechenden Gründungsprojekte tatsächlich auch gefördert werden können. Angesichts der jüngst zu beobachtenden Dynamisierung der österreichischen Start-Up Szene (vor allem im Bereich IT/Mobile) könnte sich diese Situation in Zukunft noch verschärfen. Eine Anhebung der Mittel für das JITU-Programm ist daher vor dem Hintergrund der Evaluierungsergebnisse argumentierbar. Dabei sollte auch sichergestellt werden, dass die nötigen personellen Kapazitäten innerhalb der aws zur Betreuung auf gleichem qualitativen Niveau wie bislang vorhanden sind.

- Hinsichtlich der Programmgestaltung/-organisation ist zur Steigerung der Abwicklungseffizienz und zur Vermeidung von zu großen Zeitverzögerungen zwischen Förderzusage und Auszahlung eine Umstellung der derzeit jährlichen Budgetierungsperiode auf einen Mehrjahreszeitraum (drei bis 
fünf Jahre) vorteilhaft. Die Abwicklungseffizienz ließe sich zudem auch durch eine Ausweitung der operativen Entscheidungsautonomie der aws bei Abänderungen von Standardverträgen steigern.

- Die Förderentscheidung erfolgt auf Basis einschlägiger Gutachten von Seiten der aws Experten derzeit durch ein Gremium, das zwar teilweise aus Fachexperten besteht, wobei deren Expertise nicht immer den jeweiligen Themen der Förderansuchen entsprechen. Eine thematische Trennung zwischen Life Sciences und IKT \& Physical Sciences ist zu empfehlen. Zudem sollten diese Gremien sollten in Zukunft in regelmäßigen zeitlichen Abständen (bspw. quartalsweise) abgehalten werden, um einen kontinuierlichen Fluss der Förderabwicklung garantieren zu können.

- Von Seiten der geförderten Unternehmen wird das derzeit quartalsmäßige Berichtswesen als zeitliche und organisatorische Belastung empfunden. Hier wäre eine Umstellung auf eine halbjährliche Berichterstattung anzudenken, wobei dies vor allem für noch sehr kleine Unternehmen angebracht wäre.

- Zur Verbesserung des begleitenden Programm-Monitorings wird empfohlen, dass die im Jahr 2013 begonnenen Bemühungen einer strukturierten und quantitativen Erfassung der Entwicklung der im Portfolio befindlichen Unternehmen weitergeführt bzw. ausgeweitet wird. So ließen sich Zielindikatoren wie bspw. die Überlebensquote detaillierter erfassen.

- $\quad$ Ein verstärktes statistisches Tracking der JITU geförderten Unternehmen wäre auch von Seiten der Statistik Austria über eine Aufnahme bzw. gesonderte Ausweisung der (aws und FFG) geförderten High-Tech Start-Ups Österreichs in relevanten Erhebungen (Leistungs- und Strukturerhebung, F\&EErhebung, Unternehmensdemographie, Innovationserhebung) zu erreichen. Ein solches Unterfangen wäre über die Transparenzdatenbank für die Statistik Austria technisch machbar.

- Neben dem JITU-Programm war es auch Aufgabe das Programm i2 - Business Angels zu evaluieren. Die Ergebnisse dieser Evaluierung zeigen eine positive Entwicklung dieses Programms. Insbesondere in den letzten drei Jahren kam es zu einer Übererfüllung der quantitativen Zielindikatoren. Es konnte mittlerweile eine kritische Masse an Business Angels aktiviert werden, was wiederum bereits positive Effekte auf die Zahl der erfolgreichen Vermittlungen zeitigt. Ein konkreter Handlungsbedarf ist nicht gegeben. 


\section{Referenzen}

Ács, Z. J., Autio, E. and Szerb, L. (2014): National Systems of Entrepreneurship: Measurement issues and policy implications. Research Policy 43, 476-494.

Audretsch, D. B. (1995): Innovation and Industry Evolution. Cambridge, Mass., MIT.

aws/erp-fonds (2012): Leistungsdaten Technologie \& Innovation 2011. Leistungsbericht der Austria Wirtschaftsservice Gesellschaft $\mathrm{mbH}$.

Birch D.L. (1981), Who Creates Jobs? In: The Public Interest, 65, S. 3-14.

Criscuolo, C., Gal, P. N. and Menon, C. (2014): The Dynamics of Employment Growth: New Evidence from 18 Countries. OECD Science, Technology and Industry Policy Papers, No. 14, OECD Publishing.

Duruflé, G. (2010): Government involvement in the venture capital industry - International comparisons. Canada's Venture Capital \& Private Equity Association.

EBAN (2013): European Angel Investment Overview 2012, Brussels.

Fritsch, M. (2008): Die Arbeitsplatzeffekte von Gründungen: Ein Überblick über den Stand der Forschung, Zeitschrift für ArbeitsmarktForschung, Vol. 41(1), 55-69.

Gassler, H., Reiner, C., Streicher, G. (2011): Regionalwirtschaftliche Effekte der vom Wiener AplusB-Zentrum INiTS betreuten Gründungen. Joanneum Research, Wien.

Gassler, H., Ecker, B., Reiner, C., Streicher, G., Mayer, S, Fischl, I. und Streicher, J. (2011): Zwischenevaluierung der aws-Technologieprogramme 2007-2010. Joanneum Research und KMUFA im Auftrag des BMWFJ.

Gassler, H., Ploder, M. und Kleb, U. (2013): Innovative High-Tech Gründungen - Prozesse und Monitoring. Eine Analyse des Portfolios der von der aws im Seed- und PreSeed-geförderten High-TechGründungen. Joannum Research.

Gerken, L. C. (2014): The Little Book of Venture Capital Investing: Empowering Economic Growth and Investment Portfolios. John Wiley \& Sons.

Hölzl, W., Huber, P., Kaniovski, S., Peneder, M. (2007): WIFO Weißbuch: Gründungen, Schließungen und Entwicklungen von Unternehmen: Evidenz für Österreich, WIFO Monatsberichte, No. 3/2007, Wien.

Jörg, L., Mahlich, J. und Ohler, F. (2002): Evaluierung von i2. Technopolis, Studie im Auftrag des Bundesministeriums für Wirtschaft und Arbeit.

Jörg, L., Schibany, A., Nones, B. und Gassler, H. (2006): Zwischenevaluierung der awsTechnologieprogramme, Technopolis und Joanneum Research im Auftrag des Bundesministeriums für Wirtschaft und Arbeit Abteilung C1/11 - Innovation und Transfer.

Knoll, N. (2013): Was bedeutet Effizienz der Förderungspolitik? Drei Ansatzpunkte zur Diskussion einer komplexen Frage, erschienen in: Bauer, H., Biwald, P., Pittlik, H. (Hrsg.), (2013), Zur Effizienz der Förderpolitik im Bundesstaat, (71-77), NWV, Wien.

Lerner, J. (2010): The future of public efforts to boost entrepreneurship and venture capital. Small Business Economics 35: 255-264.

OECD (2014): Policy Implications of OECD Experience in Seed and Early Stage Finance, OECD, Paris, DSTI/Ind(2014)5. 
Ruhnka, J. and Young, J. (1987): A Venture Capital Model of the Development Process for New Ventures. Journal of Business Venturing 2(2):167-184.

Schibany, A., Gassler, H. und Sellner, R. (2013): Gründungen und Unternehmensdynamik: Eine empirische und vergleichende Analyse. IHS Studie im Auftrag des erp-Fonds, Wien.

Schramm, C. (2006): The Entrepreneurial Imperative, Harper Collins Publishers, New York.

Sohl, J. (2013): “The Angel Investor Market in 2012: A Moderating Recovery Continues”. Center for Venture Research, April 25, 2013.

Sohl, J. (2014): "The Angel Investor Market in 2013: A Return to Seed Investing". Center for Venture Research, April 30, 2014.

Wilson, K. and F. Silva (2013), "Policies for Seed and Early Stage Finance: Findings from the 2012 OECD Financing Questionnaire", OECD Science, Technology and Industry Policy Papers, No. 9, OECD Publishing. http://dx.doi.org/10.1787/5k3xqsf00j33-en. 


\section{Annex}

Tabelle 12: Entwicklung PreSeed nach Schwerpunkt, 2007-2013

\begin{tabular}{c|rrrr}
\multicolumn{3}{|c}{ IKT \& Physical Science } & \multicolumn{2}{c}{ Life Science Austria (LISA) } \\
Jahr & Beschlossene Projekte & Förderungszusagen in EUR & Beschlossene Projekte & Förderungszusagen in EUR \\
\hline 2007 & 13 & $1,278,000$ & 0 & 0 \\
2008 & 11 & $1,077,000$ & 6 & 536,580 \\
2009 & 15 & $2,100,000$ & 4 & 407,000 \\
2010 & 14 & $2,644,712$ & 7 & $1,336,000$ \\
2011 & 15 & $2,195,000$ & 3 & 600,000 \\
2012 & 19 & $2,580,000$ & 8 & $1,565,600$ \\
2013 & 13 & $1,800,000$ & 10 & $1,890,800$ \\
\hline Summe & 100 & $13,674,712$ & 38 & $6,335,980$ \\
\hline \hline
\end{tabular}

Quelle: aws, eigene Darstellung.

Tabelle 13: Entwicklung Seedfinancing nach Schwerpunkt, 2007-2013

\begin{tabular}{c|rrrr} 
& \multicolumn{2}{c}{ IKT \& Physical Science } & \multicolumn{2}{c}{ Life Science Austria (LISA) } \\
Jahr & Beschlossene Projekte & Förderungszusagen in EUR & Beschlossene Projekte & Förderungszusagen in EUR \\
\hline 2007 & 0 & - & 0 & 0 \\
2008 & 10 & $6,400,000$ & 5 & $3,550,000$ \\
2009 & 7 & $4,500,000$ & 4 & $2,900,000$ \\
2010 & 3 & $2,100,000$ & 4 & $3,600,000$ \\
2011 & 11 & $6,100,000$ & 4 & $3,100,000$ \\
2012 & 10 & $6,260,000$ & 8 & $6,050,000$ \\
2013 & 21 & $12,100,000$ & 5 & $3,325,000$ \\
\hline Summe & 62 & $37,460,000$ & 30 & $22,525,000$ \\
\hline \hline
\end{tabular}

Quelle: aws, eigene Darstellung.

Tabelle 14: Annahmequoten, 2007-2013

\begin{tabular}{|c|c|c|c|c|c|c|c|c|c|}
\hline \multirow[b]{2}{*}{ Jahr } & \multicolumn{3}{|c|}{ IKT\&PHS PreSeed } & \multicolumn{3}{|c|}{ IKT\&PHS Seedfinancing } & \multicolumn{3}{|c|}{ LISA insgesamt } \\
\hline & Anfragen & Projekte & Quote & Anfragen & Projekte & Quote & Anfragen & Projekte & Quote \\
\hline 2007 & 46 & 13 & $28 \%$ & 33 & 0 & $0 \%$ & 48 & 0 & $0 \%$ \\
\hline 2008 & 36 & 11 & $31 \%$ & 36 & 10 & $28 \%$ & 40 & 11 & $28 \%$ \\
\hline 2009 & 51 & 15 & $29 \%$ & 38 & 7 & $18 \%$ & 54 & 8 & $15 \%$ \\
\hline 2010 & 65 & 14 & $22 \%$ & 31 & 3 & $10 \%$ & 64 & 11 & $17 \%$ \\
\hline 2011 & 41 & 15 & $37 \%$ & 38 & 11 & $29 \%$ & 51 & 7 & $14 \%$ \\
\hline 2012 & 81 & 19 & $23 \%$ & 40 & 10 & $25 \%$ & 48 & 16 & $33 \%$ \\
\hline 2013 & 90 & 13 & $14 \%$ & 46 & 21 & $46 \%$ & 43 & 15 & $35 \%$ \\
\hline Summe & 410 & 100 & $24 \%$ & 262 & 62 & $24 \%$ & 348 & 68 & $20 \%$ \\
\hline
\end{tabular}

Quelle: aws, eigene Darstellung. 
Tabelle 15: PreSeed Gründungen nach Entscheidungsjahr und Programmtyp

\begin{tabular}{|c|c|c|c|c|c|}
\hline Typ & Entscheidungsjahr & Gründung & noch keine Gründung & Insgesamt & Quote \\
\hline \multirow[t]{4}{*}{ PSI } & 2006 & 6 & 4 & 10 & $60,0 \%$ \\
\hline & 2008 & 14 & 2 & 16 & $87,5 \%$ \\
\hline & 2010 & 11 & 3 & 14 & $78,6 \%$ \\
\hline & 2011 & 11 & 4 & 15 & $73,3 \%$ \\
\hline \multirow[t]{4}{*}{ PSL } & 2008 & 4 & 2 & 6 & $66,7 \%$ \\
\hline & 2009 & 3 & 1 & 4 & $75,0 \%$ \\
\hline & 2010 & 6 & 1 & 7 & $85,7 \%$ \\
\hline & 2011 & 2 & 1 & 3 & $66,7 \%$ \\
\hline PSC & 2010 & 11 & 11 & 22 & $50,0 \%$ \\
\hline Alle & & 114 & 56 & 170 & $67,1 \%$ \\
\hline
\end{tabular}

Quelle: aws, eigene Darstellung.

Tabelle 16: Entwicklung Seedfinancing, 2007-2013

\begin{tabular}{rrrr} 
Jahr & Unternehmen & Mitarbeiter & Umsatz in Mio. Euro \\
\hline 1995 & 71 & 188 & 14.1 \\
1996 & 82 & 212 & 16.0 \\
1997 & 89 & 260 & 33.8 \\
1998 & 93 & 403 & 46.6 \\
1999 & 102 & 578 & 52.5 \\
2000 & 109 & 1275 & 75.9 \\
2001 & 117 & 1551 & 101.3 \\
2002 & 126 & 1672 & 101.9 \\
2003 & 136 & 1554 & 105.2 \\
2004 & 144 & 1735 & 112.6 \\
2005 & 155 & 1822 & 116.8 \\
2006 & 168 & 1916 & 121.7 \\
2007 & 171 & 2027 & 150.6 \\
2008 & 187 & 2225 & 178.8 \\
2009 & 198 & 2191 & 186.8 \\
2010 & 203 & 2342 & 207.4 \\
2011 & 218 & 2441 & 210.6 \\
2012 & 235 & 2402 & 226.1 \\
2013 & 260 & 2448 & 241.0 \\
\hline & & &
\end{tabular}

Quelle: aws, eigene Darstellung. 
Tabelle 17: Durchschnittliche zugesagte Förderhöhe, 2007-2013

\begin{tabular}{c|cr|cc}
\multicolumn{2}{c}{ PreSeed } & \multicolumn{2}{c}{ Seedfinancing } \\
Jahr & \multicolumn{1}{c}{ IKT\&PHS } & \multicolumn{1}{c}{ LISA } & \multicolumn{1}{c}{ IKT\&PHS } & LISA \\
\hline 2007 & 98,308 & - & 640,000 & 710,000 \\
2008 & 97,909 & 89,430 & 642,857 & 725,000 \\
2009 & 140,000 & 101,750 & 700,000 & 900,000 \\
2010 & 188,908 & 190,857 & 554,545 & 775,000 \\
2011 & 146,333 & 200,000 & 626,000 & 756,250 \\
2012 & 135,789 & 195,700 & 576,190 & 665,000 \\
2013 & 138,462 & 189,080 & 604,194 & 750,833 \\
\hline Summe & 136,747 & 166,736 & &
\end{tabular}

Quelle: aws, eigene Darstellung.

Tabelle 18: Seedfinancing Auszahlungen, 1990-2013

\begin{tabular}{rrrrr} 
Jahr & Darlehen & JITU & Darlehen und JITU & Kumulierte Auszahlungen \\
\hline 1990 & 483,783 & - & 483,783 & 483,783 \\
1991 & 823,601 & - & 823,601 & $1,307,384$ \\
1992 & $1,097,360$ & - & $1,097,360$ & $2,404,744$ \\
1993 & $1,263,926$ & - & $1,263,926$ & $3,668,670$ \\
1994 & $1,573,367$ & - & $1,573,367$ & $5,242,037$ \\
1995 & $1,049,214$ & - & $1,049,214$ & $6,291,251$ \\
1996 & $1,965,800$ & - & $1,965,800$ & $8,257,051$ \\
1997 & $2,372,768$ & - & $2,372,768$ & $10,629,819$ \\
1998 & $4,204,123$ & - & $4,204,123$ & $14,833,943$ \\
1999 & $3,702,681$ & - & $3,702,681$ & $18,536,623$ \\
2000 & $4,040,610$ & - & $4,040,610$ & $22,577,233$ \\
2001 & $5,210,642$ & - & $5,210,642$ & $27,787,875$ \\
2002 & $3,901,673$ & - & $3,901,673$ & $31,689,548$ \\
2003 & $3,746,437$ & - & $3,746,437$ & $35,435,985$ \\
2004 & $3,583,346$ & - & $3,583,346$ & $39,019,330$ \\
2005 & $4,385,000$ & - & $4,385,000$ & $43,404,330$ \\
2006 & $6,360,000$ & - & $6,360,000$ & $49,764,330$ \\
2007 & $2,925,000$ & - & $2,925,000$ & $52,689,330$ \\
2008 & 957,500 & $2,675,000$ & $3,632,500$ & $56,321,830$ \\
2009 & 267,500 & $4,835,000$ & $5,102,500$ & $61,424,330$ \\
2010 & - & $5,869,094$ & $5,869,094$ & $67,293,424$ \\
2011 & - & $7,285,000$ & $7,285,000$ & $74,578,424$ \\
2012 & - & $7,697,500$ & $7,697,500$ & $82,275,924$ \\
2013 & - & $10,166,500$ & $10,166,500$ & $92,442,424$ \\
\hline & & & &
\end{tabular}

Quelle: aws, eigene Darstellung. 
Tabelle 19: Jährliche Auszahlungen Jitu, 2008-2013

\begin{tabular}{ccrrrrrr} 
Jahr & Insgesamt & LISA & IKT\&PHS & Summe & LISA & IKT\&PHS & Summe \\
\hline 2008 & $4,719,000$ & 280,000 & 806,500 & $1,086,500$ & $2,020,000$ & $1,612,500$ & $3,632,500$ \\
2009 & $6,504,750$ & 207,650 & $1,194,600$ & $1,402,250$ & $1,480,000$ & $3,622,500$ & $5,102,500$ \\
2010 & $8,087,211$ & 562,350 & $1,655,767$ & $2,218,117$ & $2,149,094$ & $3,720,000$ & $5,869,094$ \\
2011 & $10,193,149$ & 682,737 & $2,225,413$ & $2,908,149$ & $2,905,000$ & $4,380,000$ & $7,285,000$ \\
2012 & $10,887,372$ & $1,037,400$ & $2,152,472$ & $3,189,872$ & $3,407,500$ & $4,290,000$ & $7,697,500$ \\
2013 & $13,817,210$ & $1,345,643$ & $2,305,067$ & $3,650,710$ & $5,222,500$ & $4,944,000$ & $10,166,500$ \\
\hline
\end{tabular}

Quelle: aws, eigene Darstellung.

Tabelle 20: Kumulierte Auszahlungen seit Jitu-Programmstart, 2008-2013

\begin{tabular}{|c|c|c|c|c|c|c|c|}
\hline \multirow[b]{2}{*}{ Jahr } & \multirow[b]{2}{*}{ Insgesamt } & \multicolumn{3}{|c|}{ PreSeed } & \multicolumn{3}{|c|}{ Seedfinancing } \\
\hline & & LISA & IKT\&PHS & Summe & LISA & IKT\&PHS & Summe \\
\hline 2008 & $4,719,000$ & 280,000 & 806,500 & $1,086,500$ & $2,020,000$ & $1,612,500$ & $3,632,500$ \\
\hline 2009 & $11,223,750$ & 487,650 & $2,001,100$ & $2,488,750$ & $3,500,000$ & $5,235,000$ & $8,735,000$ \\
\hline 2010 & $19,310,961$ & $1,050,000$ & $3,656,867$ & $4,706,867$ & $5,649,094$ & $8,955,000$ & $14,604,094$ \\
\hline 2011 & $29,504,110$ & $1,732,736$ & $5,882,280$ & $7,615,016$ & $8,554,094$ & $13,335,000$ & $21,889,094$ \\
\hline 2012 & $40,391,482$ & $2,770,137$ & $8,034,752$ & $10,804,888$ & $11,961,594$ & $17,625,000$ & $29,586,594$ \\
\hline 2013 & $54,208,692$ & $4,115,780$ & $10,339,819$ & $14,455,598$ & $17,184,094$ & $22,569,000$ & $39,753,094$ \\
\hline
\end{tabular}

Quelle: aws, eigene Darstellung.

Tabelle 21: Programmentwicklung i2, 1997-2013

\begin{tabular}{|c|c|c|c|c|c|c|c|c|}
\hline Jahr & ¡2 Projektanfragen & ¡2 Listungen & i2 Vermittlungen & ¡2 Beteiligung & i2 Angels & V Quote GD & V Quote LI & LI Quote \\
\hline 1997 & 75 & 49 & 2 & 465,000 & 37 & 2.7 & 4.1 & 65.3 \\
\hline 1998 & 85 & 44 & 2 & 196,000 & 35 & 2.4 & 4.5 & 51.8 \\
\hline 1999 & 117 & 76 & 4 & 408,000 & 47 & 3.4 & 5.3 & 65.0 \\
\hline 2000 & 249 & 102 & 4 & 321,000 & 81 & 1.6 & 3.9 & 41.0 \\
\hline 2001 & 295 & 95 & 4 & 618,000 & 83 & 1.4 & 4.2 & 32.2 \\
\hline 2002 & 427 & 103 & 6 & 960,700 & 96 & 1.4 & 5.8 & 24.1 \\
\hline 2003 & 341 & 69 & 7 & $1,735,000$ & 91 & 2.1 & 10.1 & 20.2 \\
\hline 2004 & 358 & 60 & 1 & 300,000 & 90 & 0.3 & 1.7 & 16.8 \\
\hline 2005 & 318 & 77 & 6 & $1,087,000$ & 95 & 1.9 & 7.8 & 24.2 \\
\hline 2006 & 324 & 59 & 7 & $1,600,000$ & 111 & 2.2 & 11.9 & 18.2 \\
\hline 2007 & 388 & 55 & 5 & 600,000 & 127 & 1.3 & 9.1 & 14.2 \\
\hline 2008 & 355 & 54 & 6 & 565,000 & 124 & 1.7 & 11.1 & 15.2 \\
\hline 2009 & 373 & 38 & 3 & 332,000 & 120 & 0.8 & 7.9 & 10.2 \\
\hline 2010 & 275 & 30 & 4 & 542,500 & 124 & 1.5 & 13.3 & 10.9 \\
\hline 2011 & 416 & 58 & 8 & 680,000 & 140 & 1.9 & 13.8 & 13.9 \\
\hline 2012 & 479 & 61 & 10 & 600,000 & 179 & 2.1 & 16.4 & 12.7 \\
\hline 2013 & 457 & 60 & 18 & $1,760,000$ & 205 & 3.9 & 30.0 & 13.1 \\
\hline Summe & 5332 & 1090 & 97 & $12,770,200$ & 1785 & 1.8 & 8.9 & 20.4 \\
\hline
\end{tabular}

Quelle: aws, eigene Darstellung. 
Tabelle 22: Gelistete Portfoliounternehmen i2, 2012

\begin{tabular}{lrr} 
Branchen & Portfolio gelistete Unternehmen & Prozent \\
\hline IT, Software & 25 & $25 \%$ \\
Internet, Mobile & 20 & $20 \%$ \\
Maschinenbau, Bautechnik & 18 & $18 \%$ \\
Konsum & 15 & $15 \%$ \\
Physik, Elektrotechnik & 12 & $12 \%$ \\
Cleantech, Chemie & 7 & $7 \%$ \\
Medizintechnik, Lifescience & 3 & $3 \%$ \\
\hline Summe & 100 & $100 \%$ \\
\hline
\end{tabular}

Quelle: aws, eigene Darstellung.

Tabelle 23: Regionale Verteilung der i2-Listungen, 2012

\begin{tabular}{lrrrrr} 
& $\mathbf{2 0 1 1}$ & $\mathbf{2 0 1 2}$ & $\mathbf{2 0 1 3}$ & Summe & Prozent \\
\hline W & 21 & 18 & 21 & 60 & $34 \%$ \\
OÖ & 2 & 11 & 14 & 27 & $15 \%$ \\
STMK & 9 & 11 & 8 & 28 & $16 \%$ \\
NÖ & 9 & 9 & 5 & 23 & $13 \%$ \\
SBG & 3 & 1 & 3 & 7 & $4 \%$ \\
T & 7 & 5 & 3 & 15 & $8 \%$ \\
KTN & 2 & 0 & 2 & 4 & $2 \%$ \\
DEU & 0 & 2 & 2 & 4 & $2 \%$ \\
VBG & 1 & 0 & 1 & 2 & $1 \%$ \\
CH & 0 & 0 & 1 & 1 & $1 \%$ \\
BGLD & 2 & 2 & 0 & 4 & $2 \%$ \\
ITA & 2 & 0 & 0 & 2 & $1 \%$ \\
KRO & 0 & 1 & 0 & 1 & $1 \%$ \\
SLO & 0 & 1 & 0 & 1 & $1 \%$ \\
\hline Summe & 58 & 61 & 60 & 179 & $100 \%$ \\
\hline
\end{tabular}

Quelle: aws, eigene Darstellung. 

AutorInnen: Helmut Gassler und Richard Sellner

Titel: Programmevaluierung Jitu und i2 - Business Angels

Projektbericht/Research Report

(C) 2014 Institute for Advanced Studies (IHS),

Stumpergasse 56, A-1060 Vienna • ㅍㅛㅛ +43 $159991-0 \bullet$ Fax +43 1 59991-555 • http://www.ihs.ac.at 